\title{
Solute Carrier Transporters as Potential Targets for the Treatment of Metabolic Disease
}

\author{
Tina Schumann, Jörg König, Christine Henke, Diana M. Willmes, Stefan R. Bornstein, Jens Jordan, Martin F. Fromm, \\ and Andreas L. Birkenfeld
}

Section of Metabolic and Vascular Medicine, Medical Clinic III, Dresden University School of Medicine (T.S., C.H., D.M.W., S.R.B.), and Paul Langerhans Institute Dresden of the Helmholtz Center Munich at University Hospital and Faculty of Medicine (T.S., C.H., D.M.W.), Technische Universität Dresden, Dresden, Germany; Deutsches Zentrum für Diabetesforschung e.V., Neuherberg, Germany (T.S., C.H., D.M.W., A.L.B.); Clinical Pharmacology and Clinical Toxicology, Institute of Experimental and Clinical Pharmacology and Toxicology,

Friedrich-Alexander-Universität Erlangen-Nürnberg, Erlangen, Germany (J.K., M.F.F.); Institute for Aerospace Medicine, German Aerospace Center and Chair for Aerospace Medicine, University of Cologne, Cologne, Germany (J.J.); Diabetes and Nutritional Sciences, King's College London, London, United Kingdom (S.R.B., A.L.B.); Institute for Diabetes Research and Metabolic Diseases of the Helmholtz Centre Munich at the University of Tübingen, Tübingen, Germany (A.L.B.); and Department of Internal Medicine, Division of Endocrinology, Diabetology and Nephrology, Eberhard Karls University Tübingen, Tübingen, Germany (A.L.B.)

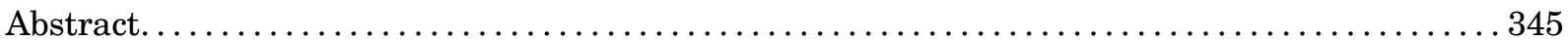

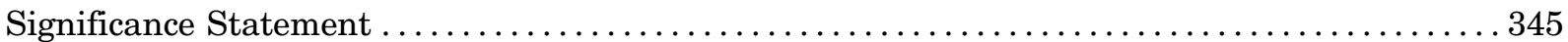

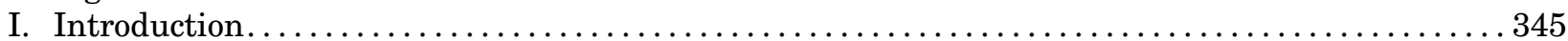

II. Solute Carrier Membrane Transport Proteins . . . . . . . . . . . . . . . . . . . . . . . . . . . . 346

A. Definitions: Solute Carrier Superfamily and Other Membrane Transporters............. 346

B. Solute Carrier-Related Human Diseases ................................... 346

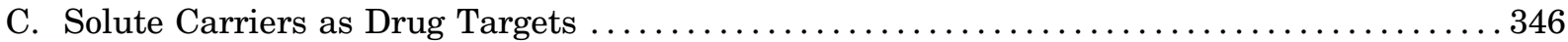

1. Solute Carrier 5A1/Sodium-Glucose Cotransporter 1 and Solute Carrier 5A2/

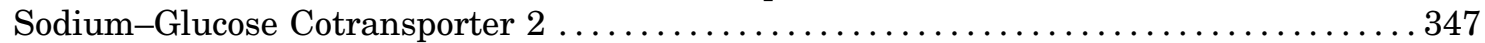

III. Links to Human Metabolic Disease and Potential Targets ......................... 348

A. Solute Carrier 13: The Sodium-Sulfate/Carboxylate Cotransporter Family . . . . . . . . . . . . 348

1. Solute Carrier 13A5/Sodium-Coupled Citrate Transporter ..................... 348

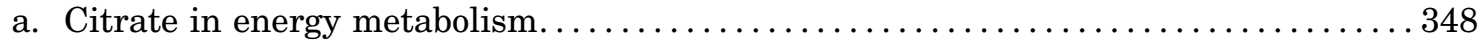

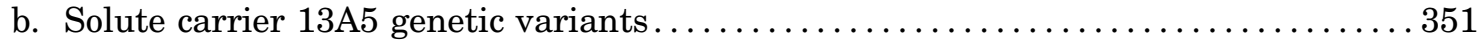

c. Metabolic phenotype of animal models................................ 351

d. Pharmacological inhibition of sodium-coupled citrate transporter............... 352

2. Therapeutic Potential of Solute Carrier 13 Transporters...................... 354

B. Solute Carrier 16: The Monocarboxylate Transporter Family $\ldots \ldots \ldots \ldots \ldots \ldots \ldots \ldots \ldots \ldots 4$

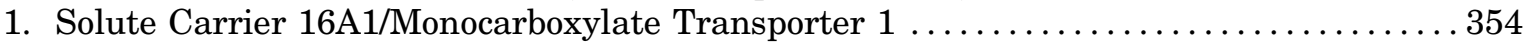

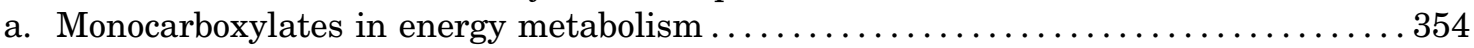

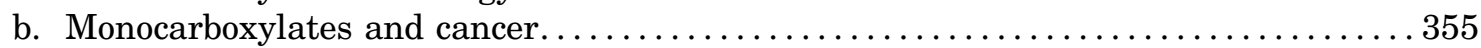

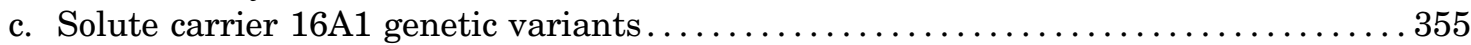

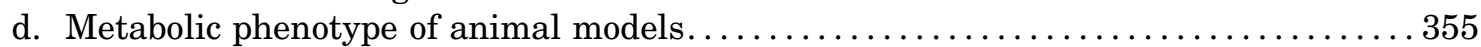

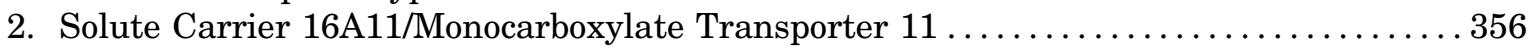

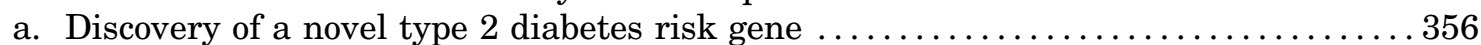

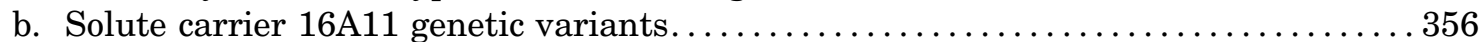

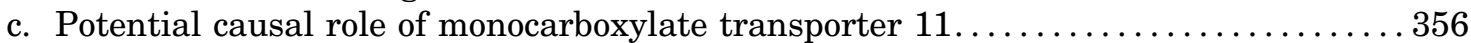

d. Metabolic phenotype of animal models................................ 357

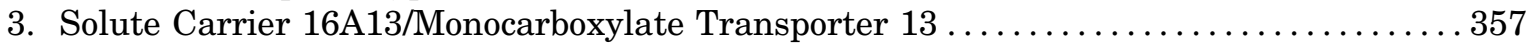

4. Therapeutic Potential of Solute Carrier 16 Transporters...................... 358

Address correspondence to: Dr. Andreas L. Birkenfeld, Department of Internal Medicine, Division of Endocrinology, Diabetology and Nephrology, Eberhard Karls University Tübingen, Otfried-Müller-Straße 10, 72076 Tübingen, Germany. E-mail: Andreas.Birkenfeld@ med.uni-tuebingen.de

https://doi.org/10.1124/pr.118.015735.

This work was funded by grants from the German Research Foundation to A.L.B. (BI1292/4-2; BI1292/10-1; BI1292/12-1) and A.L.B. and S.R.B. (IRTG2251). 
C. Solute Carrier 25: The Mitochondrial Carrier Family ........................ 358

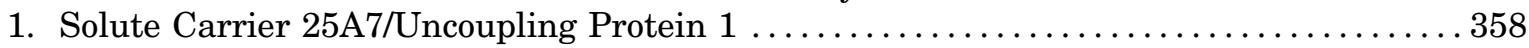

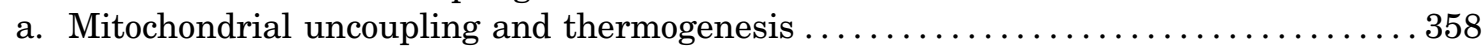

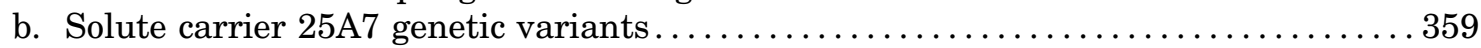

c. Metabolic phenotype of animal models............................... 359

d. Mitochondrial uncoupling to treat metabolic disease ...................... 359

2. Solute Carrier 25A8/Uncoupling Protein 2 and Solute Carrier 25A9/Uncoupling

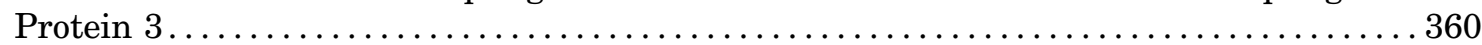

a. Physiological functions irrespective of mitochondrial uncoupling. . . . . . . . . 360

b. Solute carrier $25 \mathrm{~A} 8$ and solute carrier $25 \mathrm{~A} 9$ genetic variants $\ldots \ldots \ldots \ldots \ldots \ldots \ldots \ldots$

c. Metabolic phenotype of animal models................................ 361

3. Solute Carrier 25A13/Aspartate-Glutamate Carrier $2 \ldots \ldots \ldots \ldots \ldots \ldots \ldots \ldots \ldots \ldots \ldots \ldots \ldots \ldots \ldots$

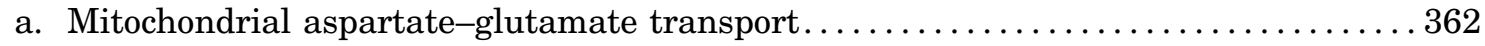

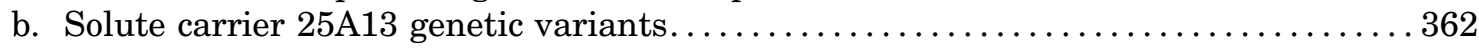

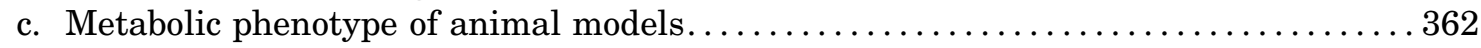

4. Solute Carrier 25A20/Carnitine-Acylcarnitine Carrier....................... 363

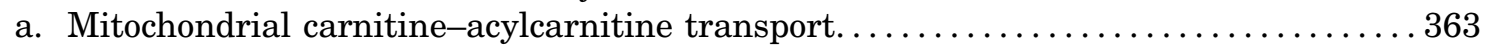

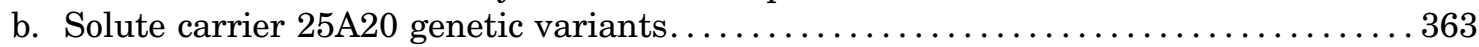

5. Solute Carrier 25A24/ATP-Mg/Phosphate Carrier Protein 1 . . . . . . . . . . . . . . 363

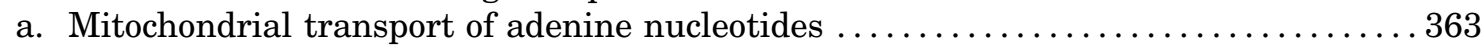

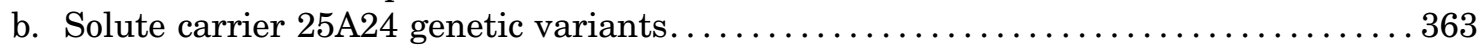

c. Metabolic phenotype of animal models............................... 363

6. Therapeutic Potential of Solute Carrier 25 Transporters...................... 364

D. Solute Carrier 30: The Zinc Transporter Family ............................. 364

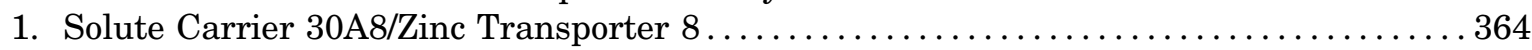

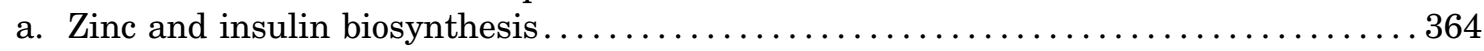

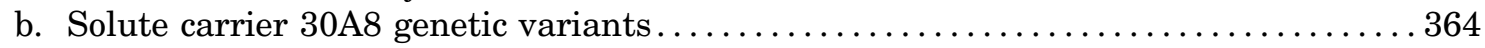

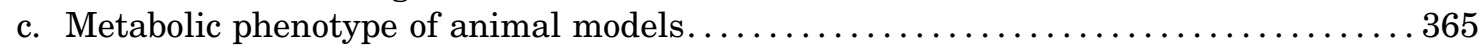

d. Pharmacological activation or inhibition of zinc transporter $8 \ldots \ldots \ldots \ldots \ldots \ldots \ldots 6$

2. Therapeutic Potential of Solute Carrier 30 Transporters. . . . . . . . . . . . . . 366

IV. Proposed Requirements for Metabolic Target Structures.......................... 367

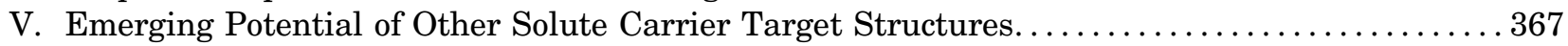

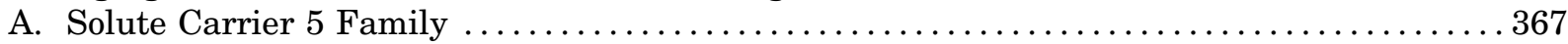

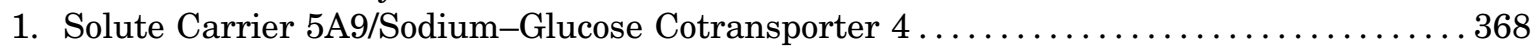

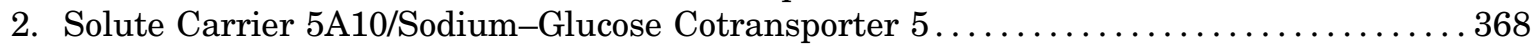

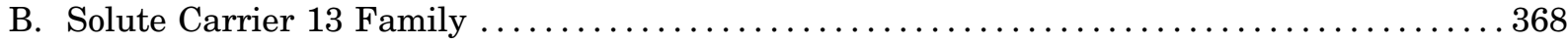

1. Solute Carrier 13A1/Sodium-Coupled Sulfate Cotransporter $1 \ldots \ldots \ldots \ldots \ldots \ldots \ldots$

2. Solute Carrier 13A2/Sodium-Coupled Di- and Tricarboxylate Transporter $1 \ldots \ldots \ldots 368$

3. Solute Carrier 13A3/Sodium-Coupled Di- and Tricarboxylate Transporter 3 ......... 369

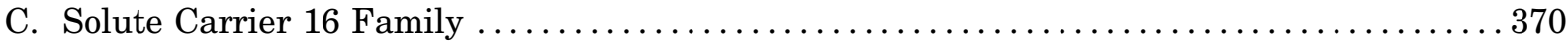

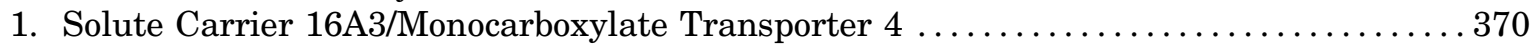

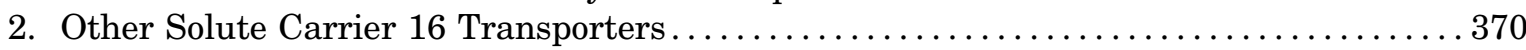

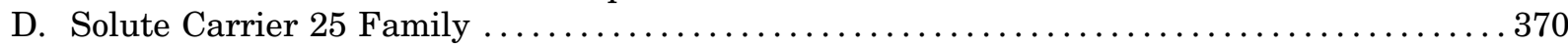

1. Solute Carrier 25A25/ATP-Mg/Phosphate Carrier Protein $3 \ldots \ldots \ldots \ldots \ldots \ldots \ldots \ldots 370$

2. Solute Carrier 25A40/Mitochondrial Carrier Family Protein .................. 371

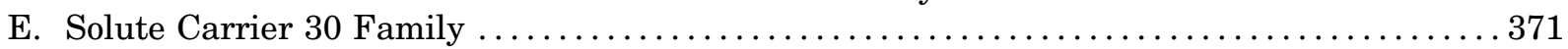

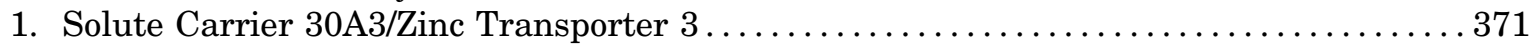

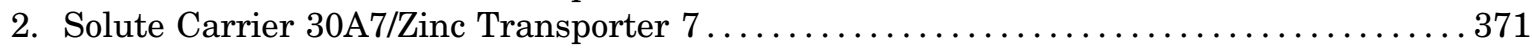

\footnotetext{
ABBREVIATIONS: AGC2, aspartate-glutamate carrier 2; APC, ATP-Mg/phosphate carrier protein; BMI, body mass index; CAC, carnitine-acylcarnitine carrier; CTLN2, adult-onset type II citrullinemia; DAG, diacylglycerol; FDA, Food and Drug Administration; GWAS, genome-wide association study; HFD, high-fat diet; HGNC, Human Genome Organization Gene Nomenclature Committee; MCFP, mitochondrial carrier family protein; MCT, monocarboxylate transporter; MR, Mendelian randomization; NaC, sodium-carboxylate transporter; $\mathrm{NaCT}$, sodium-coupled citrate transporter; NaDC, sodium-coupled di- and tricarboxylate transporter; NAFLD, nonalcoholic fatty liver disease; $\mathrm{NaS}$, sodium-coupled sulfate cotransporter; NICCD, neonatal intrahepatic cholestasis by citrin deficiency; PPAR $\alpha$, peroxisome proliferatoractivated receptor $\alpha$; SGLT, sodium-glucose cotransporter; SLC, solute carrier; SNP, single-nucleotide polymorphism; T2D, type 2 diabetes; TAG, triacylglycerol; TCA, tricarboxylic acid; UCP, uncoupling protein; ZnT, zinc transporter.
} 
3. Other Solute Carrier 30 Transporters ................................ 371

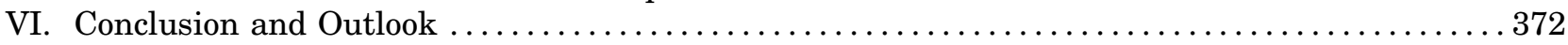

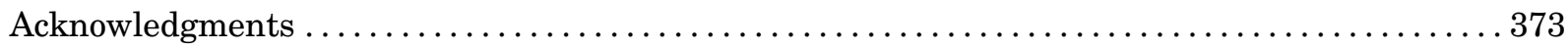

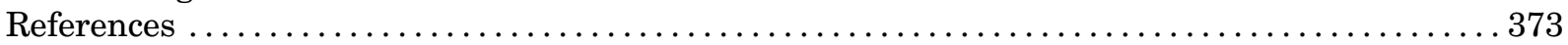

Abstract_-The solute carrier (SLC) superfamily comprises more than 400 transport proteins mediating the influx and efflux of substances such as ions, nucleotides, and sugars across biological membranes. Over 80 SLC transporters have been linked to human diseases, including obesity and type 2 diabetes (T2D). This observation highlights the importance of SLCs for human (patho)physiology. Yet, only a small number of SLC proteins are validated drug targets. The most recent drug class approved for the treatment of T2D targets sodium-glucose cotransporter 2 , product of the SLC5A2 gene. There is great interest in identifying other SLC transporters as potential targets for the treatment of metabolic diseases. Finding better treatments will prove essential in future years, given the enormous personal and socioeconomic burden posed by more than 500 million patients with T2D by 2040 worldwide. In this review, we summarize the evidence for SLC transporters as target structures in metabolic disease. To this end, we identified SLC13A5/sodium-coupled citrate transporter, and recent proof-of-concept

\section{Introduction}

Metabolic processes convert nutrients to energy and build up proteins, lipids, carbohydrates, and nucleic acids, also implicating the chemical reactions that facilitate these processes, such as the intra- and intercellular transport of substances. Thus, metabolism maintains cell structure and function and keeps the cell and the whole organism alive. Dysregulation of these pathways, whether through genetic and/or environmental factors, can drive metabolic diseases. The most common metabolic disease is type 2 diabetes (T2D), posing a pandemic health threat with 425 million affected people worldwide. T2D damages the heart, brain, blood vessels, eyes, kidney, and nerves, thereby increasing the risk of premature death. Obesity is the major risk factor for T2D and is associated with ectopic fat accumulation in the liver [nonalcoholic fatty liver disease (NAFLD)] and insulin resistance, a pathological state characterized by the inability of insulin to properly coordinate glucose homeostasis. Overnutrition and an unhealthy diet coupled with a sedentary lifestyle dramatically increase the prevalence of obesity, fatty liver, insulin resistance, and T2D, particularly in genetically predisposed individuals. Despite modern therapies, including lifestyle intervention, medication, and surgery, patients with T2D exhibit two to three times increased mortality (Engelmann et al., 2016). Approximately 1.6 million people die each year from diabetes sequelae, particularly cardiovascular disease (www.who.int). Antihyperglycemic treatments reduce microvascular complications. Metformin is generally studies confirm its therapeutic potential in T2D and nonalcoholic fatty liver disease. Further SLC transporters were linked in multiple genome-wide association studies to T2D or related metabolic disorders. In addition to presenting better-characterized potential therapeutic targets, we discuss the likely unnoticed link between other SLC transporters and metabolic disease. Recognition of their potential may promote research on these proteins for future medical management of human metabolic diseases such as obesity, fatty liver disease, and T2D.

Significance Statement-Given the fact that the prevalence of human metabolic diseases such as obesity and type 2 diabetes has dramatically risen, pharmacological intervention will be a key future approach to managing their burden and reducing mortality. In this review, we present the evidence for solute carrier (SLC) genes associated with human metabolic diseases and discuss the potential of SLC transporters as therapeutic target structures. recommended as first-line therapy in patients with T2D. Evidence from recent cardiovascular outcome trials led to the recommendation by the American Diabetes Association and the European Association for the Study of Diabetes to prescribe sodium-glucose cotransporter (SGLT)2 inhibitors or glucagon-like peptide 1 receptor agonists as preferred second medication in patients with T2D and established cardiovascular disease (Davies et al., 2018). Thus, novel drug targets may beget therapies that further reduce diabetes complications, which are urgently needed in the face of the T2D pandemic.

Genome-wide association studies (GWAS) or Mendelian randomization (MR) studies can be used to identify new therapeutic targets in human beings. These approaches reveal single-nucleotide polymorphisms (SNPs) as risk factors associated with human diseases. Up to now, GWAS and other sequencing studies have linked more than 140 gene loci to T2D. Of these, 15 loci cover genes encoding putative or known membrane transport proteins (Morris, 2018). Membrane transporters are crucial for cellular homeostasis and serve as cellular gatekeepers by controlling the trafficking of molecules such as sugars, amino acids, fatty acids, nucleotides, inorganic ions, and drugs (Hediger et al., 2004). Through selective transport across biological membranes, cells are able to adapt to metabolic challenges, which is critical for the maintenance of physiological processes. Indeed, about 2000 human genes-equivalent to about $10 \%$ of all human genes-are predicted to be related to membrane transport proteins (Hediger 
et al., 2004). Functional gene variants of transport proteins are involved in human diseases, and about $10 \%$ of established T2D loci are related to membrane transporters.

\section{Solute Carrier Membrane Transport Proteins}

Solute carrier (SLC) proteins are a group of transmembrane transporters that mediate solute influx and efflux across plasma and intracellular membranes (Hediger et al., 2004). The SLC nomenclature system was introduced in the 1990s by Matthias A. Hediger and Phyllis McAlpine, the first chair of the Human Genome Organization Gene Nomenclature Committee (HGNC). An overview of all currently known SLC genes is provided in the SLC tables (slc.bioparadigms.org) and the HGNC database (www.genenames.org/cgi-bin/ genefamilies/set/752). Both overlap; however, some entries differ (Perland and Fredriksson, 2017). The publication of the first mini-review on SLCs presented 298 genes grouped in 43 SLC families (Hediger et al., 2004). At present, it comprises over 60 families with more than 400 genes, and new transporter genes continue to be discovered and added to the SLC superfamily. The ongoing SLC research has yielded hitherto unknown contributions to health and disease, making them increasingly important as drug targets.

\section{A. Definitions: Solute Carrier Superfamily and Other Membrane Transporters}

Membrane transporter classification into SLCs and other transport proteins-so-called non-SLCs-was introduced in 2004. The classification within the SLC transporter superfamily is based exclusively on biological function and not on sequence homology. Members of the same SLC transporter family that share similar substrates show an at least $20 \%$ protein sequence identity (Hediger et al., 2004).

The two main membrane transport protein classes are channels and carriers. Carriers feature substratebinding sites that undergo conformational changes to move substrates across membranes and transportdefined substrate quantities per translocation cycle. Conversely, substrates passively flow through channels. Carriers are classified into active and passive transporters (Hediger, 1994). SLCs mediate passive and secondary active substrate transport. Passive or facilitated transport (uniport) does not require energy input because solutes are transported along an electrochemical gradient. Substance transport against electrochemical gradients requires energy-providing mechanisms and is mediated by active transporters that are divided into primary and secondary active transporters. Secondary active transporters facilitate substance transfer against an electrochemical gradient. The process is coupled with ion movements down an electrochemical gradient, thus providing energy for active substance transport. When substance and driving ion are transferred in the same direction, the transporter is termed a cotransporter (symporter), whereas exchangers (antiporter) translocate molecules in opposite directions.

The SLC superfamily includes passive transporters as well as secondary active transporters, whereas nonSLC transporters include channels and primary active transporters (Hediger et al., 2004). In contrast to carriers, channels mediate ion or solute flow at high rates controlled by gating mechanisms, that is, pore opening and closing. Primary active transporters are ATP dependent and include the family of ATP-binding cassette transporter and ATPases (ion pumps). Both transporters transfer their substrates out of cells or into cellular organelles and bind or hydrolyze ATP to transport substances such as ions, lipids, carbohydrates, xenobiotics, and drugs (ATP-binding cassette transporter), or transport ions coupled to ATP hydrolysis (ATPases).

\section{B. Solute Carrier-Related Human Diseases}

The important role of SLC transporters in metabolism is reflected by their expression profile, with high levels in metabolically active organs such as the liver, kidney, brain, and intestine, and by links with human diseases. SLC transporter defects contribute to Mendelian and complex multifactorial diseases. SLC-related diseases range from neurological disorders associated with neurotransmitter transporter dysfunction of the SLC1 or SLC6 family such as amyotrophic lateral sclerosis, Parkinson disease, Alzheimer's disease, epilepsy, schizophrenia, or autism (Pramod et al., 2013; Grewer et al., 2014), to less common diseases such as Bartter and Gitelman syndrome, nephropathic conditions caused by mutations in SLC12 genes encoding renal cation-coupled chloride transporters (Hebert et al., 2004). More general overviews on SLC transporters in human diseases and their potential as drug targets have been published recently (Rask-Andersen et al., 2013; Lin et al., 2015; Zhang et al., 2019). In contrast, our review focuses on SLC transporters that are linked to metabolic diseases and therefore presents promising novel drug targets with which to treat $\mathrm{T} 2 \mathrm{D}$ and associated metabolic disorders.

\section{Solute Carriers as Drug Targets}

Transport proteins are, besides receptors and enzymes, the third most common class of pharmacological targets, with 67 target genes corresponding to $15 \%$ of all human drug targets (Rask-Andersen et al., 2011). Currently, over 900 transporter-encoding genes are listed in the HGNC dataset. With over $40 \%$ of these genes, the SLC superfamily encodes the largest group of membrane transporters in humans (Hediger et al., 2013). 
Despite their important physiological functions and known links to human diseases-over 80 SLC transporters have been implicated in genetic disorders (Lin et al., 2015)—only a small number of approved drugs target SLC transporters. Compared with other classes of human drug targets, the SLC superfamily appears understudied in relation to drug target discovery, potentially for a number of reasons: SLCs have only recently been characterized, and the area is still evolving. A lack of structural characterization of human SLC membrane proteins impedes drug design. Moreover, SLC transporters fulfill essential functions and are broadly expressed and redundant in their substrate specificity, making a specific pharmacological intervention even more challenging (Rask-Andersen et al., 2013).

Over 30 SLC proteins have recently been presented as current, prospective, or potential drug targets (RaskAndersen et al., 2013; Lin et al., 2015; Zhang et al., 2019). More stringent analyses revealed 12 drug classes, including those proposed in the recent reviews, that are approved by the Food and Drug Administration (FDA) and whose primary mode of action is mediated selectively through one SLC or nonselectively through at least two SLCs (César-Razquin et al., 2015). The drug classes target the SLC5, SLC6, SLC12, SLC18, SLC22, SLC25, and SLC29 families. Six additional drugs targeting SLC5, SLC6, SLC9, SLC10, SLC22, and SLC40 family members are being tested in phase II clinical trials or beyond (César-Razquin et al., 2015). Importantly, the SLC family is expected to be generally druggable using small-molecule compounds, and about $75 \%$ of SLCs are predicted to bind small organic molecules (César-Razquin et al., 2015). In general, most drugs use known binding sites; it is clear that the higher the similarity between compound and endogenous ligands, the higher the probability of affecting the biological action of the transporter (Fauman et al., 2011). Indeed, for the majority of well-studied SLCs, small-molecule inhibitors have already been reported (César-Razquin et al., 2015). Further elucidation of physiological functions and three-dimensional SLC structures will provide new opportunities for drug development. However, membrane proteins are difficult to crystallize. It is hoped that new strategies for membrane protein crystallization and computational analyses methods will reveal high-resolution human SLC structures that can guide site-directed mutagenesis to uncover substrate-binding sites and functionally important domains. Ultimately, structure-based drug design may foster the translation of potential SLC targets into clinical practice.

1. Solute Carrier 5A1/Sodium-Glucose Cotransporter 1 and Solute Carrier 5A2/Sodium-Glucose Cotransporter 2. The successful clinical introduction of SGLT2 inhibitors is a prime example for the emerging potential of SLC transporters as drug targets. SGLT1 and SGLT2, encoded by the $S L C 5 A 1$ and $S L C 5 A 2$ genes, respectively, are members of the SGLT family. Both transporters are crucial for glucose reabsorption in proximal renal tubules. Loss-of-function mutations in SLC5A1 are linked to glucose-galactose malabsorption in humans, a condition characterized by severe watery diarrhea in newborns (Lindquist and Meeuwisse, 1962; Turk et al., 1991; Wright et al., 2001). Patients with glucosegalactose malabsorption are lifelong glucose and galactose intolerant, but can lead a normal life on a sugar-free diet. The finding that SLC5A2 mutations cause familial glucosuria implicated the transporter in glucose homeostasis (Santer et al., 2000, 2003; van den Heuvel et al., 2002). Affected individuals exhibit urinary glucose excretion up to $>100 \mathrm{~g} /$ day in the face of euglycemia without renal tubular dysfunction. The condition is considered relatively benign because it rarely leads to hypoglycemia.

The discovery that phlorizin, a naturally occurring compound isolated from apple tree bark, lowers blood glucose levels through SGLT inhibition was key to the development of the class of SGLT inhibitors. Phlorizin was shown to inhibit human renal glucose reabsorption in the 1930s (Chasis et al., 1933). Studies in the 1980s showed that the compound restores normal glucose levels in partially pancreatectomized rats and corrected the insulin secretion defect induced by hyperglycemia (Rossetti et al., 1987; Kahn et al., 1991). Eventually, the beneficial response was attributed to dual SGLT1 and SGLT2 inhibition (Toggenburger et al., 1982; Ehrenkranz et al., 2005). Glucose reabsorption from the tubular lumen into the cell through SGLTs is driven by an ATPase-generated sodium concentration gradient. After absorption by SGLTs, glucose exits the cell through SLC family 2-facilitated glucose transport member 2 , entering the bloodstream and contributing to glycemic variance. SGLT2 reclaims more than $90 \%$ of filtered glucose (Vallon et al., 2011). Glucose, which is not reabsorbed via SGLTs, is excreted in urine, which is promoted by SGLT inhibition and counteracts hyperglycemia.

Gastrointestinal side effects such as nausea and vomiting, and pharmacokinetic issues limiting oral dosing halted the clinical development of phlorizin as an oral antidiabetic agent (Ehrenkranz et al., 2005). Meanwhile, other SGLT-targeting compounds have been generated and approved for the treatment of T2D. Currently, four selective SGLT2 inhibitors are FDA-approved for mono- and combination therapy: canagliflozin, dapagliflozin, empagliflozin, and ertugliflozin (Fioretto et al., 2015; Scheen, 2015; Shubrook et al., 2015; Triplitt and Cornell, 2015; Derosa and Maffioli, 2018). Others are in the pipeline and may be approved in the near future, such as sotagliflozin (McCrimmon and Henry, 2018). Of the four FDAapproved drugs, empagliflozin has the highest selectivity for SGLT2 compared with SGLT1, whereas 
canagliflozin is the least selective (Hsia et al., 2017). In phase II and phase III clinical trials, SGTL2 inhibition reduced glycated hemoglobin, $\mathrm{HbA}_{1 \mathrm{c}}$, in patients with diabetes by $0.5 \%$ to $1 \%$. It also produces modest weight loss, reduces arterial blood pressure, induces glucosuria with osmotic diuresis and natriuresis, and reduces intrahepatic lipid content (Fioretto et al., 2015; Scheen, 2015; Shubrook et al., 2015; Triplitt and Cornell, 2015; Derosa and Maffioli, 2018; Kahl et al., 2019). In large-scale international, multicenter cardiovascular safety trials, including the EMPA-REG OUTCOME, the CANVAS Program, and DECLARE-TIMI 58, SGLT2 inhibition reduced cardiovascular endpoints (Zinman et al., 2015; Neal et al., 2017; Wiviott et al., 2019). In a metaanalysis of the three trials, SGLT2 inhibition reduced major adverse cardiovascular events, with benefits restricted to patients with atherosclerotic cardiovascular disease (Zelniker et al., 2019). Overall, proven benefits in cardiovascular outcome trials with a relatively benign safety profile support the use of the SGLT2 inhibitors in the treatment of patients with T2D and cardiovascular disease, heart failure, and diabetic kidney disease (Davies et al., 2018). More recent trials also establish the role of SGLT2 inhibitors in the treatment of type 1 diabetes (Mathieu et al., 2018; Rosenstock et al., 2018).

\section{Links to Human Metabolic Disease and Potential Targets}

From a pharmacological perspective, membrane transporters have the potential to fulfill two important functions, as follows: they can directly serve as drug targets, or deliver drugs modulating other cellular targets (Hediger et al., 2004). In this review, we focus on drug targets, which in functional and/or genetic studies have been previously linked to human metabolic diseases. The metabolic phenotypes of interest comprise obesity, NAFLD, insulin resistance, and T2D. Furthermore, our main goal was to characterize physiology and pathophysiology associated with SLCs rather than detailed transporter structure and transport mechanism.

Importantly, we evaluated the therapeutic potential of selected family members on the basis of animal and human investigations. For some SLCs, the link to human metabolic disease and the rationale of modulating its activity for therapeutic purposes are strong. For example, GWAS of larger populations, wellcharacterized inherited disorders, and/or valid mouse models may have yielded promising data, supporting the role of the SLC in the development, prevention, or cure of metabolic disease. Such investigational targets are presented in this section. Table 1 displays an overview of SLCs that we consider conclusive candidates for clinical development as well as SLCs targeted by already approved drugs such as SGLT2. For these transporters, Figs. 1 and 2 display their cellular localization and tissue expression. Moreover, we present other selected members that do not currently have a fully convincing data set because existing association studies are lacking or only performed in small populations, disease patterns are not closely linked or are less well studied in terms of metabolic disease, or are only supported by mouse data. These candidates are promising for future research, and are presented in Emerging Potential of Other Solute Carrier Target Structures and summarized in Table 2.

\section{A. Solute Carrier 13: The Sodium-Sulfate/ Carboxylate Cotransporter Family}

The tricarboxylic acid (TCA) cycle is essential for energy synthesis under aerobic conditions. It provides substrates and thereby regulates multiple metabolic reactions, including glucose and lipid metabolism. TCA cycle intermediates such as citrate, succinate, and $\alpha$-ketoglutarate are transported across the plasma membrane into the cell by sodium $\left(\mathrm{Na}^{+}\right)$-coupled anion transporters of the SLC13 family, regulating levels of these metabolites in plasma, urine, and tissue. These transporters are ubiquitously expressed, but primarily in the liver, kidney, small intestine, and brain (Pajor, 2014). The human SLC13 family consists of five genes-SLC13A1, SLC13A2, SLC13A3, SLC13A4, and $S L C 13 A 5$ - and is divided into two functionally unrelated groups, as follows: the sodium-coupled diand tricarboxylate transporters $(\mathrm{NaDC})$, also termed sodium-carboxylate transporters $(\mathrm{NaC})$, including NaDC1/NaC1 (SLC13A2), NaDC3/NaC3 (SLC13A3), and $\mathrm{NaC2}$, also sodium-coupled citrate transporter (NaCT) (SLC13A5), carry TCA cycle intermediates; the other two family members, sodium-coupled sulfate transporters (NaS)1 (SLC13A1) and NaS2 (SLC13A4), show distinct substrate specificities and mainly transport sulfate, selenite, and thiosulfate (Markovich and Murer, 2004). The structure and function of the SLC13 transporter have been reviewed in detail (Markovich and Murer, 2004; Pajor, 2006, 2014; Bergeron et al., 2013).

\section{Solute Carrier 13A5/Sodium-Coupled Citrate} Transporter.

a. Citrate in energy metabolism. NaCT, encoded by the SLC13A5 gene, is the sodium-coupled citrate transporter. At physiological $\mathrm{pH}, \mathrm{NaCT}$ is selective for citrate over the other TCA cycle intermediates succinate, malate, and fumarate, which are transported with lower affinities (Inoue et al., 2002a,b, 2004). NaCT provides TCA cycle intermediates for the liver and brain, metabolically active tissue, where $\mathrm{NaCT}$ is predominantly expressed (Figs. 1 and 2). In the liver, NaCT affects mitochondrial energy production, fatty acid synthesis, cholesterol synthesis, cytosolic glycolysis, and gluconeogenesis (Inoue et al., 2004). In the brain, $\mathrm{NaCT}$ is primarily expressed in neural plasma membranes and enables, in concert with $\mathrm{NaDC} 3$, an anaplerotic supply of TCA cycle intermediates (Wada et al., 2006; 


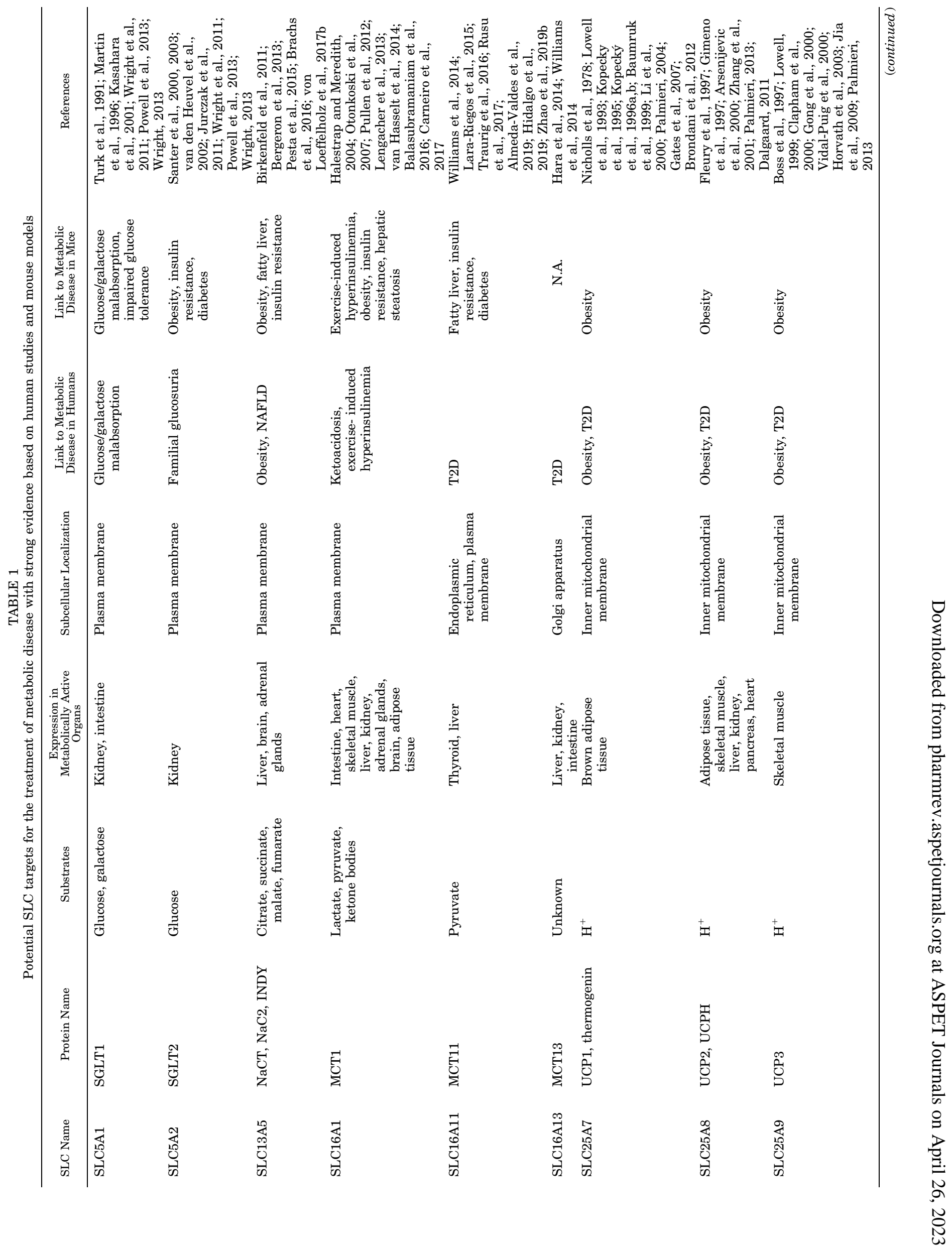




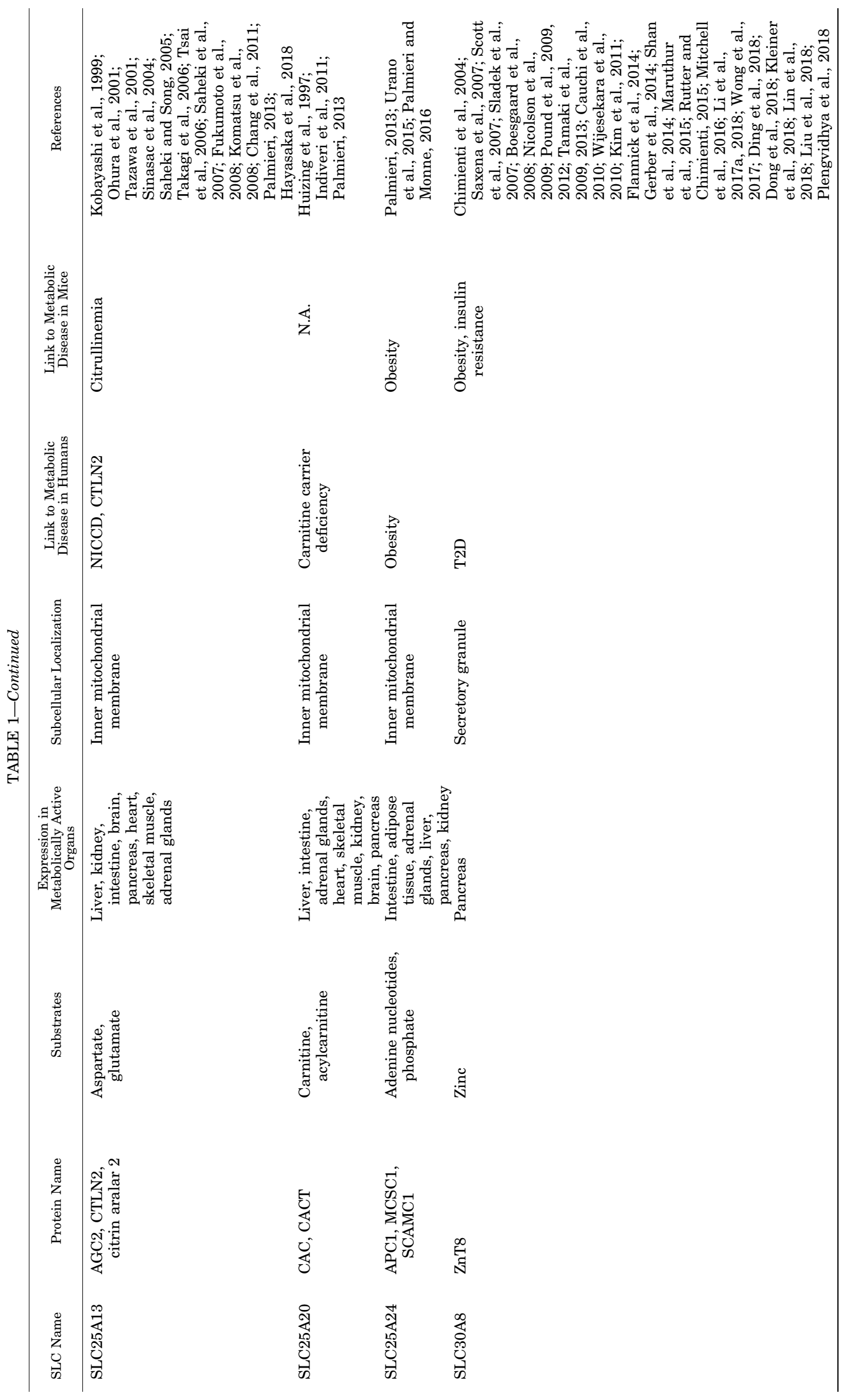




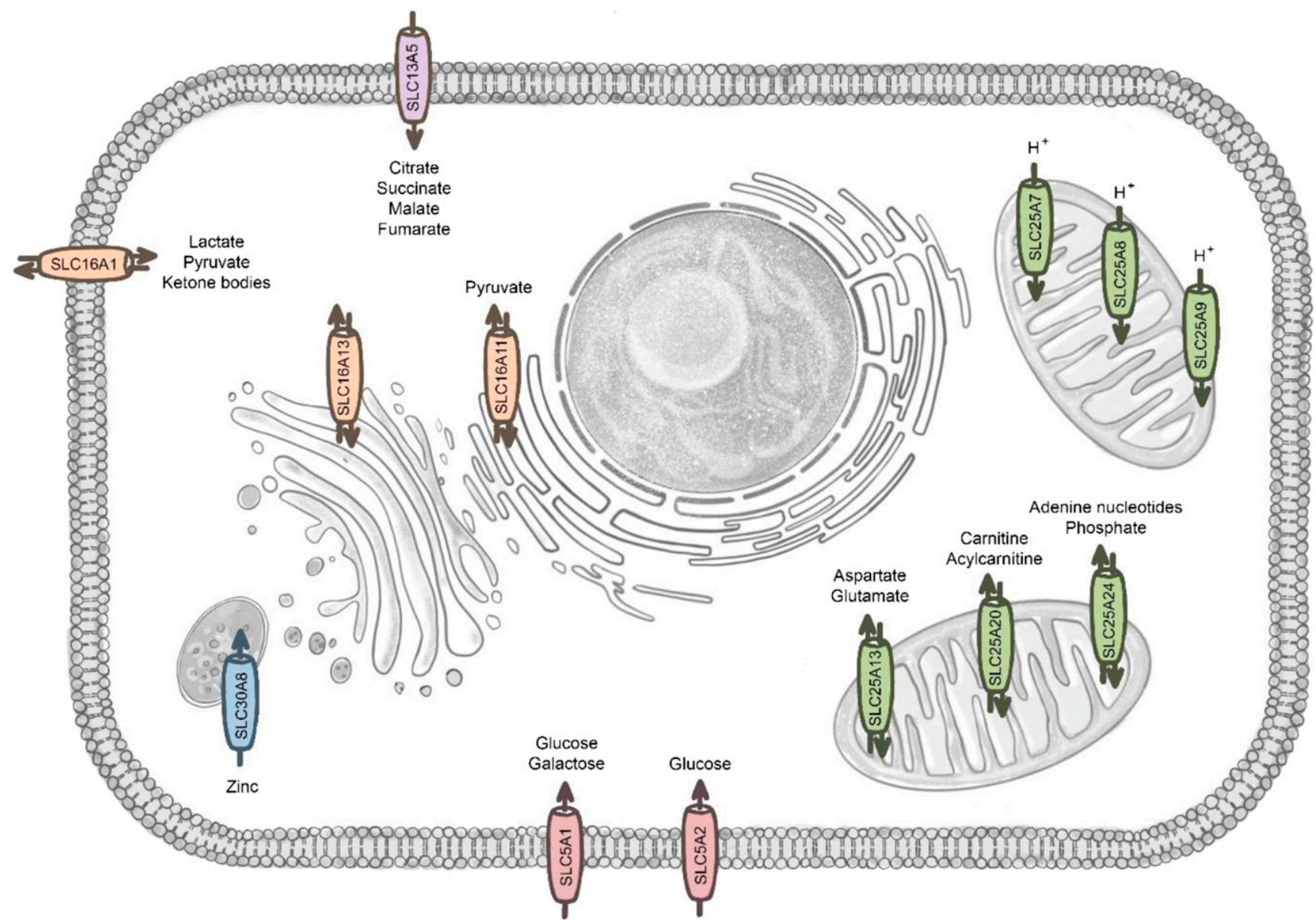

Fig. 1. Cellular localization of potential SLC targets for the treatment of metabolic disease. SLC transporters are expressed in the plasma membrane or intracellular membranes. Of the five presented SLC families, which are displayed in different colors, 13 members have been assessed to exhibit strong evidence of association with metabolic disease based on human studies and mouse models. The equal shapes of the transporter symbols do not correspond to the different modes of transport, but the arrows indicate transport direction, for example, whether the transporter is proposed to mediate influx of its substrates into the cell (SLC5A1, SLC5A2, SLC13A5) or intracellular compartments (SLC25A7, SLC25A8, SLC25A9, SLC30A8), facilitates both influx and efflux (SLC16A1, SLC16A11, SLC16A13), or acts as an exchanger (SLC25A13, SLC25A20, SLC25A24). The main substrates of the transporters are indicated next to the transporter symbol, except for SLC16A13, which is not characterized at all concerning substrate specificity and transport mode.

Yodoya et al., 2006). NaCT (also INDY, acronym for "I'm Not Dead Yet") was first identified in Drosophila melanogaster, where mutations in the mammalian $\mathrm{NaCT}$ homolog Indy lead to life span extension (Rogina et al., 2000). Moreover, Indy mRNA was shown to be downregulated in diet-restricted healthy flies, and Indy-mutated long-lived flies share several phenotypes with long-lived caloric-restricted flies. Therefore, $\mathrm{NaCT}$ has been proposed to regulate the aging processes at least in part by activating an AMP-activated protein kinase/peroxisome proliferator-activated receptor $\gamma$ coactivator $1 \alpha$ network (Rogina et al., 2000; Neretti et al., 2009; Wang et al., 2009; Rogers and Rogina, 2014; Schwarz et al., 2015).

b. Solute carrier $13 A 5$ genetic variants. SLC13A5 mutations have been described recently to cause earlyonset epileptic encephalopathy (Thevenon et al., 2014; Hardies et al., 2015; Klotz et al., 2016; Schossig et al., 2017; Weeke et al., 2017). In contrast, NaCT has not been directly linked to metabolic disease in humans, and genome-wide association is lacking. However, in liver samples of obese, insulin-resistant patients with NAFLD, SLC13A5 mRNA expression was significantly increased and associated with hepatic steatosis (von Loeffelholz et al., 2017b). Importantly, the study is the first hint of a relationship between $\mathrm{NaCT}$ and metabolic disease in humans.

c. Metabolic phenotype of animal models. The association between $\mathrm{NaCT}$ and metabolic disease is well characterized in animal models (Table 1). Slc13a5 deletion in mice resulted in a metabolic phenotype resembling caloric restriction (Birkenfeld et al., 2011). Remarkably, Slc13a5 knockout mice were protected from high-fat diet (HFD)-induced and aging-induced obesity, hepatic steatosis, and insulin resistance. The phenotype was linked to an increase in energy expenditure, hepatic mitochondrial biogenesis, and fatty acid oxidation, and a reduction in hepatic lipogenesis (Birkenfeld et al., 2011). Consistent with these mouse data, the knockdown of human SLC13A5 resulted in 


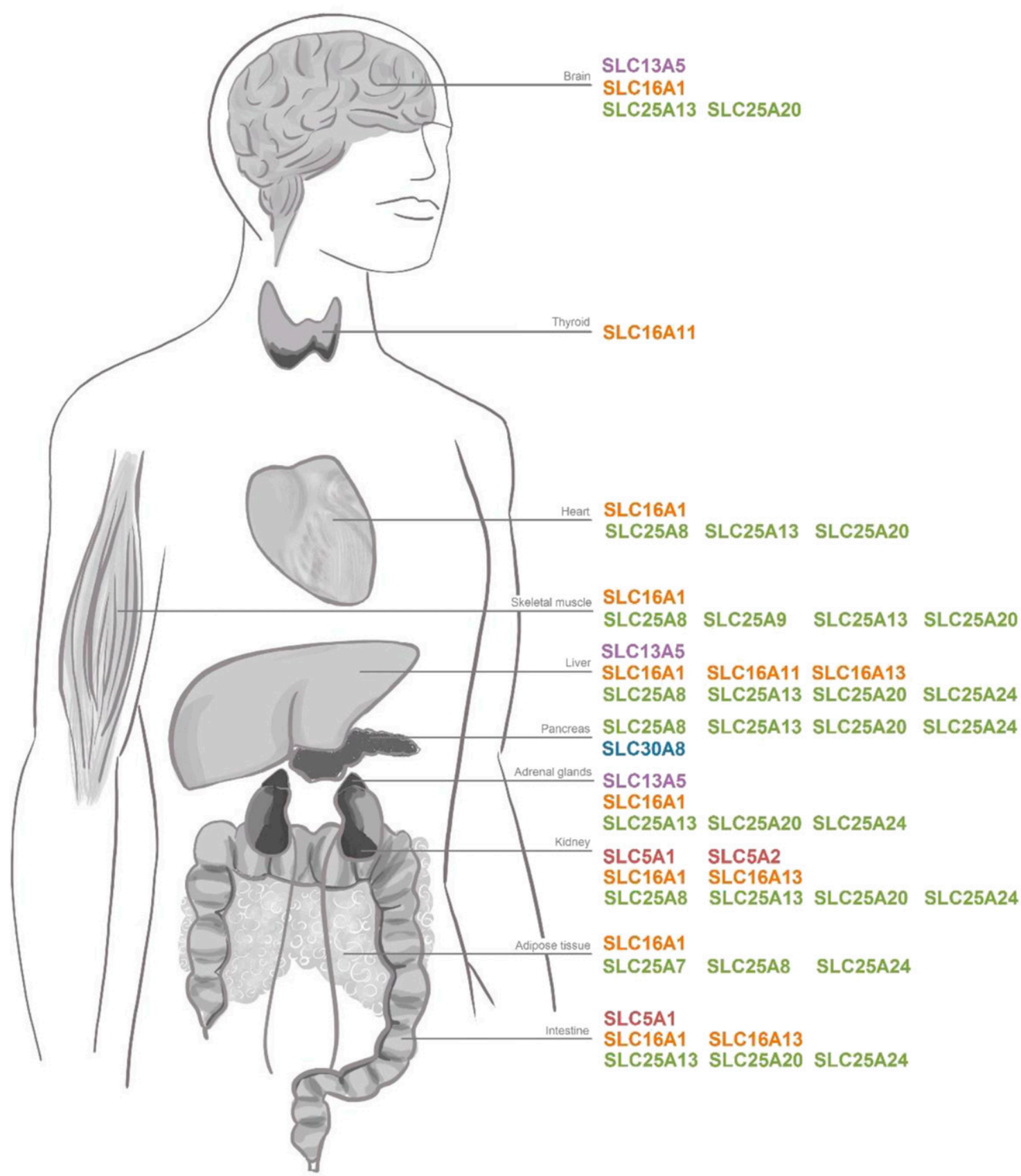

Fig. 2. Tissue distribution of potential SLC targets for the treatment of metabolic disease. SLC transporters are broadly expressed. The 13 SLCs, which belong to five families displayed in different colors and were evaluated to have strong potential as targets for the treatment of metabolic disease, are highly expressed in metabolically active organs such as the liver, kidney, brain, and intestine.

lower lipid levels in a hepatocarcinoma cell line ( $\mathrm{Li}$ et al., 2015). Moreover, data obtained from rat primary hepatocytes show an increase in NaCT-mediated citrate transport after glucagon treatment and additionally detect cAMP-responsive element-binding proteinbinding sites within the Slc13a5 promotor, suggesting that Slc13a5 is a cAMP-responsive element-binding protein-dependent glucagon target that is induced during periods of fasting and in T2D (NeuschäferRube et al., 2014).

d. Pharmacological inhibition of sodium-coupled citrate transporter. Slc13a5 deletion and pharmacological inhibition ameliorate obesity, insulin resistance, and NAFLD in various model organisms (Birkenfeld et al., 2011; Shulman and Helfand, 2011; Willmes and Birkenfeld, 2013; Neuschäfer-Rube et al., 2014; Huard et al., 2015; Pesta et al., 2015; Brachs et al., 2016; Willmes et al., 2016, 2018; Rogina, 2017; von Loeffelholz et al., 2017a). NaCT has therefore been proposed as a promising target for the prevention and treatment of metabolic diseases. In rats, the inducible hepatic Slc13a5 knockdown at onset of the HFD feeding using 2'-O-methoxyethyl chimeric antisense oligonucleotides improved hepatic glucose production 


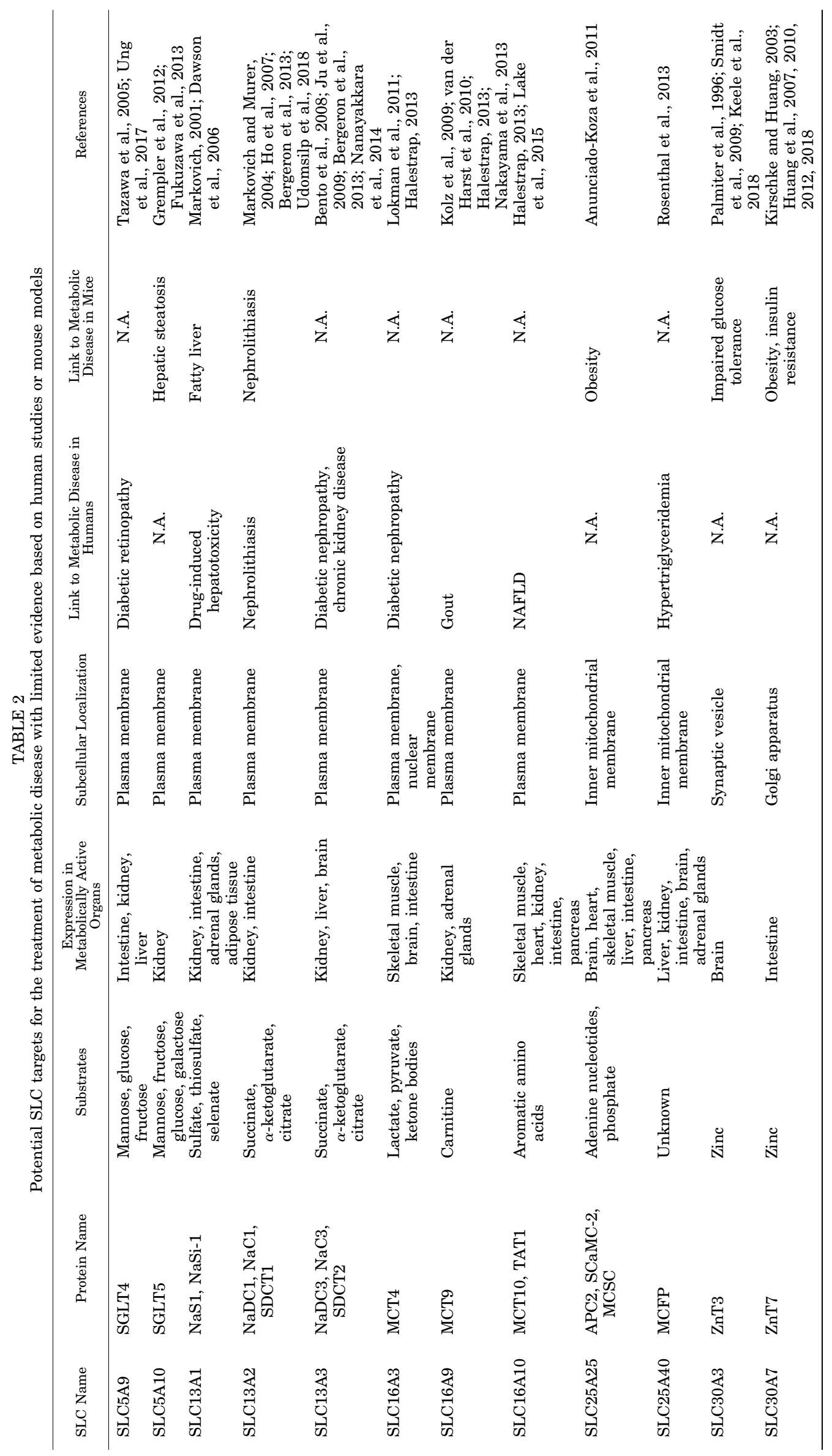


and insulin sensitivity and protected from NAFLD (Pesta et al., 2015), thus reproducing the Slc13a5 knockout phenotype. Moreover, hepatic Slc13a5 knockdown via small interfering RNA improved insulin sensitivity and hepatic triglyceride accumulation in mice (Brachs et al., 2016). These data led to the development of specific small-molecule NaCT inhibitors (Pajor and Randolph, 2007; Sun et al., 2010).

A first proof-of-concept study applied compound 2 (PF-06649298), a small dicarboxylate molecule that was discovered using a substrate-based design strategy and was shown to selectively bind to the NaCT transporter in a competitive and stereosensitive manner. Impressively, compound 2 led to the potent inhibition of NaCT-mediated citrate transport in vitro and in vivo (Huard et al., 2015). In compound 2-treated mice, glucose intolerance on HFD was completely reversed and hepatic di- and triglyceride content tended to be reduced (Huard et al., 2015). This finding suggests an increase in $\beta$-oxidation, supported by a tendency of higher $\beta$-hydroxybutyrate level. To determine the residues that are involved in binding of compound 2, methods of molecular modeling and site-directed mutagenesis, transport characterization, and cell surface biotinylation of the human $\mathrm{NaCT}$ transporter were used (Pajor et al., 2016). Apparently, residues adjacent to the putative citrate-binding site (G228, V231, V232, and G409) affect citrate transport and its inhibition, but also residues located outside the putative citrate-binding site (Q77 and T86) might be relevant (Pajor et al., 2016). Another small-molecule NaCT inhibitor, compound 4a, is less selective, but also reduced citrate uptake and moderately ameliorated glucose metabolism in rodents (Huard et al., 2016). Finally, these data reveal new mechanistic insights and could guide SLC13 inhibitor design.

To sum up, cell-based studies and mouse and human data indicate the potential of targeting the citrate transporter $\mathrm{NaCT}$ to ameliorate metabolic disease. Notably, small-molecule NaCT inhibitors are already developed and validated in mouse models of dietinduced obesity.

2. Therapeutic Potential of Solute Carrier 13 Transporters. Of the five SLC13 family members, three additional members of the SLC13 family are marginally linked to metabolic disorders (Table 2) and will be presented in Emerging Potential of Other Solute Carrier Target Structures. However, whereas loss of SLC13A1/NaS1 function in mice causes a complex phenotype, SLC13A2/NaDC1 and SLC13A3/NaDC3 may be linked to renal disease rather than T2D. Summarizing the data of the SLC13 sodium-coupled di- and tricarboxylate cotransporters (Tables 1 and 2), SLC13A5/NaCT is the most attractive candidate with which to target obesity and related diseases. SLC13A5 variants have not been linked to human metabolic diseases in genetic association studies; however, the
Slc13a5 knockout mouse phenotype and studies with selective small-molecule $\mathrm{NaCT}$ inhibitors reflect its ability to protect from metabolic diseases such as obesity, fatty liver, insulin resistance, and T2D.

\section{B. Solute Carrier 16: The Monocarboxylate Transporter Family}

Monocarboxylates are major players in cellular energy metabolism and are substrates as well as products of anabolic and catabolic pathways in various tissues. The transport of monocarboxylates in and out of cells is mediated by monocarboxylate transporters (MCT) of the SLC16 family, which comprises 14 members (Halestrap and Price, 1999; Halestrap and Meredith, 2004). The quantitatively most important substrate is L-lactate. Monocarboxylate transport has not yet been confirmed for all members of this family. MCT1 (SLC16A1), MCT2 (SLC16A7), MCT3 (SLC16A8), and MCT4 (SLC16A3) catalyze proton-linked transport of monocarboxylates, such as L-lactate, pyruvate, and ketone bodies across the plasma membrane in and out of cells (Halestrap and Meredith, 2004). MCT7 (SLC16A6) appears to export ketone bodies (Hugo et al., 2012), and MCT11 (SLC16A11) transports pyruvate (Rusu et al., 2017). Other family members transport larger hydrophobic monocarboxylates: MCT8 (SLC16A2) and MCT10 (SLC16A10) transport thyroid hormones and aromatic amino acids (Visser et al., 2011); MCT9 (SLC16A9) is a carnitine efflux transporter (Suhre et al., 2011); and MCT12 (SLC16A12) transports creatine (Abplanalp et al., 2013). Natural substrates of four family members remain unknown. The transporters are broadly expressed and affect energy metabolism in the liver, kidney, adipose tissue, heart, muscle, and brain. Their structure, role, and regulation have been extensively reviewed (Halestrap and Price, 1999; Halestrap and Meredith, 2004; Halestrap, 2012, 2013; Halestrap and Wilson, 2012).

\section{Solute Carrier 16A1 / Monocarboxylate Transporter} 1.

a. Monocarboxylates in energy metabolism. SLC16A1 is ubiquitously expressed and encodes for MCT1, the first and best-characterized member of the SLC16 family, which transports L-lactate, pyruvate, and ketone bodies across the plasma membrane (Halestrap and Price, 1999) (Figs. 1 and 2). Glycolytic cells produce lactic acid when oxygen is limited. Lactic acid is also a substrate for lipogenesis and gluconeogenesis in the liver, kidney, and adipose tissue, as well as a respiratory substrate for tissues such as the heart, skeletal muscle, and brain. Cells and tissues exhibiting these anabolic and catabolic pathways express high levels of MCT1, MCT2, and MCT4, catalyzing the uptake or efflux of lactic acid (Halestrap and Meredith, 2004; Halestrap, 2012, 2013; Halestrap and Wilson, 2012). This lactate shuttle between different cell types in the same tissue is well described, for 
example, in the muscle, where glycolytic white muscle fibers secrete lactate via MCT4 that is taken up by oxidative red muscle fibers via MCT1, and in the brain, where glycolytic astrocytes secrete lactate via MCT1 and MCT4 and oxidative neurons take up lactate via MCT2. Strikingly, lactate is the primary carbon source in the TCA cycle and therefore of energy production, highlighting the importance of monocarboxylates in energy homeostasis (Hui et al., 2017). Similar to lactic acid, pyruvate is produced during glycolysis, fueling gluconeogenesis or oxidation in a tissue-specific fashion. The ketone bodies $\beta$-hydroxybutyrate and acetoacetate are produced during fasting or ketogenic diets and provide energy for the brain, heart, and skeletal muscle (Halestrap and Meredith, 2004; Halestrap, 2012, 2013; Halestrap and Wilson, 2012).

b. Monocarboxylates and cancer. To date, MCT1 has primarily been linked to the pathogenesis of cancer, whereas little is known about its potential as target for other indications (Jones and Morris, 2016; Fisel et al., 2018). Because lactate metabolism is critical for tumor growth and MCT1 is an important monocarboxylate transporter in various cancers, this transporter could have relevance in anticancer therapy. The role of monocarboxylate transporters in tumor tissue has been well described: cancer cells rely on anaerobic glycolysis to supply energy (Warburg effect) and produce lactate, which is transported out of the cell via monocarboxylate transporters (Jones and Morris, 2016). As MCT1, MCT2, and MCT4 act in a proton-coupled mechanism, these carriers transport protons out of the cell, thereby attenuating intracellular acidosis. Secreted lactate is transported by SLC16 proteins into neighboring cells that rely on oxidative phosphorylation. Lactate shuttling in tumor tissue between cells with different energy needs is mediated by MCT1, MCT2, and MCT4. Therefore, these transporters may serve as tumor markers and drug targets. Of note, the MCT1 inhibitor AZD3965 is in phase I clinical testing (www.clinicaltrials.gov) as an anticancer drug. Multiple studies validated the efficacy of the therapeutic principle in cancer cell lines and an in vivo model of xenograft tumors, confirming the inhibition of bidirectional monocarboxylate transport by AZD3965 and illustrating reduced glycolytic activity and consequently reactivation of oxidative phosphorylation to maintain cell survival (Bola et al., 2014; Polański et al., 2014; Beloueche-Babari et al., 2017).

c. Solute carrier $16 A 1$ genetic variants. Using AZD3965 in treating metabolic disease is not an obvious choice because genetic proof of an association between SLC16A1 gene locus and diet-induced obesity and T2D in human subjects is lacking. Nevertheless, MCT1 is linked to metabolic traits in humans and in mice (Table 1). In two independent studies, homozygous and heterozygous SLC16A1 loss-of-function gene mutations were linked to recurrent, severe ketoacidosis in humans (van Hasselt et al., 2014; Balasubramaniam et al., 2016). Ketoacidosis is caused by excess circulating ketone bodies in the face of insulinopenia and is generally a severe complication of diabetes (Nuttall, 1965; Winegrad and Clements, 1971). Targeted exome sequencing in an index patient and her family members revealed nine rare SLC16A1 variants (van Hasselt et al., 2014). Subsequent analysis of more patients with unexplained ketoacidosis identified seven additional variants, including six truncating mutations likely resulting in loss of protein function. Mutations were correlated with disease severity and MCT1 protein expression and activity. Therefore, MCT1-mediated transport of ketone bodies into extrahepatic tissues may be essential in preventing acidosis during periods of high ketone body turnover (van Hasselt et al., 2014). Although heterozygous SLC16A1 mutations can result in clinical symptoms, heterozygous relatives of patients did not develop ketoacidosis. Additional environmental and/or genetic factors may exacerbate symptoms. The hypothesis is supported by the symptom-free intervals seen in the patients (van Hasselt et al., 2014).

Promotor-activating SLC16A1 mutations have been identified in patients with exercise-induced hyperinsulinemia, a condition characterized by periods of severe hypoglycemia after physical exercise and linked to syncope (Meissner et al., 2001; Otonkoski et al., 2003, 2007). Excess $S L C 16 A 1$ expression in pancreatic $\beta$-cells may increase circulating insulin via a mechanism linking increased pyruvate uptake and catabolism to higher ATP levels and insulin secretion (Otonkoski et al., 2007). MCT1-related pathophysiology in humans deserves further evaluation because MCT1 inhibition may be beneficial in treating exercise-induced hyperinsulinemia, but could also promote ketoacidosis.

$$
\text { d. Metabolic phenotype of animal models. }
$$
Exercise-induced hyperinsulinemia and hypoglycemia were reproduced in transgenic mice with $\beta$-cell-specific Slc16a1 overexpression (Pullen et al., 2012). Genetic Slc16a1 deletion further suggested that the transporter affects whole-body energy homeostasis (Lengacher et al., 2013). Slc16a1 null mutation in mice is embryonically lethal, whereas heterozygous animals develop normally. Interestingly, heterozygous Slc16a1 knockout mice are resistant to diet-induced obesity and show less insulin resistance and hepatic steatosis compared with littermate controls. Reduced weight gain was associated with decreased hepatic and white adipose fat accumulation, potentially mediated by AMP-activated protein kinase secondary to reduced substrate availability (Carneiro et al., 2017). Metabolic changes in MCT-deleted mice support the concept that monocarboxylate transport is important for metabolic control. A putative role of MCT1 in metabolic disease is also supported by increased brain expression in diet-induced obese and genetically obese $o b / o b$ and diabetic $d b / d b$ 
mice (Pierre et al., 2007). Chronic hyperglycemia elicits a similar response in rat brains (Canis et al., 2009), which governs energy homeostasis. Slc16a1 was also upregulated in mouse liver, kidney, and small intestine by the peroxisome proliferator-activated receptor $\alpha$ $(\operatorname{PPAR} \alpha)$, an important mediator of lipid catabolism that is linked to obesity (König et al., 2008).

In summary, multiple publications implicate monocarboxylate transporters in body weight regulation (Carneiro and Pellerin, 2015). Despite the metabolic phenotype of Slc16a1 haploinsufficient mice and altered Slc16a1 expression in obesity, MCT1 was never discussed as a metabolic treatment target, and no genetic evidence has been described in human GWAS. However, clinical testing of the MCT1 inhibitor AZD3965 holds promise.

\section{Solute Carrier 16A11/Monocarboxylate Trans-} porter 11.

a. Discovery of a novel type 2 diabetes risk gene. A hitherto unnoticed and uncharacterized member of the SLC16 family has recently attracted interest in metabolic research: MCT11, the transporter encoded by the SLC16A11 gene. SLC16A11 sequence variants predicted T2D risk in a GWAS. The SIGMA type 2 diabetes consortium analyzed SNPs in Mexicans and other Latin Americans and identified a haplotype associated with T2D (Williams et al., 2014). The risk haplotype includes one silent and four missense SNPs in the SLC16A11 gene. Three of four amino acid-substituting SNPs are predicted to be tolerated (rs117767867, rs75418188, rs75493593), whereas one $\mathrm{SNP}$ ( $r$ 13342692) is predicted to be damaging. The risk to develop T2D increases by approximately $20 \%$ per haplotype copy. Subjects carrying the risk haplotype develop T2D on average 2.1 years earlier and at $0.9 \mathrm{~kg} / \mathrm{m}^{2}$ lower body mass index (BMI) than noncarriers. The stronger association in younger and leaner individuals may reflect a role of MCT11 in T2D pathogenesis independently of adiposity. Remarkably, the risk haplotype is present in $48 \%$ of Native American and in $12 \%$ of Asian populations, but rare or absent in European or African samples. Gene expression analysis in human tissues illustrated high SLC16A11 expression in the liver, salivary glands, and thyroid (Fig. 2). Moreover, human MCT11 protein localizes to the endoplasmic reticulum (Fig. 1), but only marginally to the plasma membrane (Williams et al., 2014; Rusu et al., 2017). Metabolite profiling of SLC16A11-expressing HeLa cells showed a substantial increase in triacylglycerol (TAG) and a modest increase in diacylglycerol (DAG) concentrations, whereas lysophosphatidylcholine, cholesterol ester, and sphingomyelin lipid concentrations were decreased. Perturbed lipid metabolism, especially TAG and DAG accumulation, promotes insulin resistance and T2D. Possibly, MCT11 modulates T2D risk in part through actions on hepatic lipid metabolism. b. Solute carrier $16 A 11$ genetic variants. The findings regarding the $S L C 16 A 11$ risk haplotype in Mexicans (Williams et al., 2014) inspired subsequent investigations. The SLC16A11 polymorphism rs13342692, predicted to be the crucial SNP in the risk haplotype, was not significantly associated with T2D in Mayas (Lara-Riegos et al., 2015). However, the study presented significant associations between rs13342692 and plasma insulin levels and homeostasis model assessment for insulin resistance in nonobese nonaffected risk allele carriers, implicating a higher susceptibility to metabolic disorders for risk allele carriers. Additionally, SLC16A11 risk haplotype carriers exhibit decreased insulin action, and transaminase levels are increased in carriers with diabetes (Almeda-Valdes et al., 2019). The diabetes risk haplotype was further confirmed in a Hispanic/Latino cohort in the United States with an association to diabetes in the Mexican-American, but not in non-Mexican Hispanic/Latino groups (Hidalgo et al., 2019).

In American Indians, carriers of the SLC16A11 $r s 75493593$ risk allele with a BMI $<35 \mathrm{~kg} / \mathrm{m}^{2}$ showed an increased risk for T2D and a decreased T2D risk with higher BMI (Traurig et al., 2016). Similarly, a longitudinal follow-up on the BMI and diabetes status of individuals yielded a higher hazard ratio in the leanest BMI and vice versa. The longitudinal data set also raised the question of whether $r s 75493593$ allele carriers with lower $\mathrm{BMI}$ are prone to $\mathrm{T} 2 \mathrm{D}$, or whether they lose weight as a consequence of T2D onset. In one study cohort, individuals carrying the risk allele lost more weight after T2D onset, highlighting the possibility that additional factors influence the association between BMI and the risk allele in T2D. However, the finding was not reproduced in another study cohort (Traurig et al., 2016). Individuals carrying the risk allele displayed increased carbohydrate and reduced lipid oxidation rates (Traurig et al., 2016). Differential levels of lipid metabolites have also been observed in SLC16A11expressing HeLa cells (Williams et al., 2014). Reduced mitochondrial $\beta$-oxidation due to reduced mitochondrial density with subsequent DAG accumulation predisposes development of insulin resistance and T2D (Samuel et al., 2010; Birkenfeld and Shulman, 2014; Goedeke et al., 2019). Therefore, lower lipid oxidation rates in a SLC16A11 risk allele carrier could conceivably confer increased T2D risk. The SLC16A11 risk allele also affected expression of the adjacent RNASEK gene, encoding for the ribonuclease $\mathrm{K}$. The interaction decreases RNASEK expression in the adipose tissue, muscle, and whole blood of risk allele carriers. Possibly, both genes contribute to the phenotypic associations observed in this study. Because altered hepatic and adipose RNASEK expression were not detected in another study of risk haplotype carriers (Rusu et al., 2017), the significance of this observation is unclear.

c. Potential causal role of monocarboxylate transporter 11. Recent data showed that the SLC16A11 
diabetes risk haplotype affects MCT11 by reducing its mRNA expression, but also by inhibiting transporter translocation to the plasma membrane (Rusu et al., 2017). Specifically, SLC16A11 gene expression was reduced in liver samples taken from carriers of the risk haplotype in a gene dose-dependent manner. Additionally, the authors show that the four coding variants associated with $\mathrm{T} 2 \mathrm{D}$ risk reduce cell-membrane expression of the transporter. Diminished interaction with the chaperone protein basigin may have contributed to reduced transporter activity. Finally, to analyze the consequences of reduced MCT11 function, the authors knocked down SLC16A11 in primary human hepatocytes and observed that intracellular acylcarnitines, DAGs and TAGs, were significantly increased. DAGs and ceramides mediate insulin resistance via activation of novel protein kinases and subsequent serine phosphorylation of the insulin receptor and/or the insulin receptor substrate 1 (Birkenfeld and Shulman, 2014; Petersen et al., 2016). Thus, MCT11 may affect cellular fatty acid and lipid metabolism, thereby promoting insulin resistance and T2D. However, the exact molecular pathway remains unknown. Reduced cell surface localization of T2D risk variants may be critical in T2D pathogenesis. Despite this, only a very small portion of the wild-type protein localizes to the plasma membrane, and it is unclear whether MCT11 serves as an intracellular and/or plasma membrane transporter. MCT11 may translocate to the plasma membrane in physiological conditions requiring its transport activity. Furthermore, other SLC16 family members also transport pyruvate, and additional substrates may be transported by MCT11. Perhaps some substrates are specifically transported by MCT11 under particular physiological or metabolic conditions. If so, compensatory substrate transport by other monocarboxylate transporter may not suffice, when MCT11 function is reduced through genetic risk variants. Clearly, cellular localization and the regulation of MCT11 in health and disease require further study.

d. Metabolic phenotype of animal models. The first data on a MCT11-deficient mouse model were published in 2019 (Zhao et al., 2019b). Whereas constitutive Slc16a11 knockout did not result in severe metabolic consequences, even in animals fed HFD, reconstitution of knockout mice with mutant Slc16a11 in the liver using a liver-specific adeno-associated virus resulted in a metabolic phenotype. The phenotype comprised increased blood and liver triglycerides and reduced glucose tolerance and insulin resistance. Hepatic reconstitution of mutant Slc16a11, but not of the wildtype Slc16a11, results in Lipin-1 upregulation. Therefore, the observed increase in liver triglycerides may have been mediated through Lipin-1, an enzyme involved in triglyceride synthesis. In contrast to previous studies indicating that reduced MCT11 function promotes T2D risk (Rusu et al., 2017), the authors proposed that gain-of-function mutations in Slc16a11 foster T2D. However, functional characterization of mutant Slc16a11 was not shown, and hepatic reconstitution with wild-type Slc16a11 also resulted in lipid accumulation. Although these findings suggest that MCT11 contributes to hepatic lipid metabolism, they do not prove that gain of function explains the observed detrimental metabolic phenotype in their MCT11 mouse model.

In conclusion, although the detailed mechanism of MCT11 action in the development of T2D is still not clear, the causal role of MCT11 in T2D is strongly supported by genetic association combined with in vitro genetic and functional data predicting the loss of protein function to be disease promoting. Thus, enhancing MCT11 function may hold "the promise as a potential therapy for T2D" (Stadler and Farooqi, 2017). Therapeutic approaches could increase gene expression or protein level, restore MCT11 protein interaction with basigin for correct plasma membrane localization, or increase transport activity of the protein.

3. Solute Carrier 16A13/Monocarboxylate Transporter 13. In parallel with SLC16A11, SLC16A13, encoding for MCT13, was identified as another locus of the SLC16 family associated with T2D. The SLC16A13 polymorphism rs312457 was linked to T2D in a recent Japanese GWAS (Hara et al., 2014). The associations were not confirmed in Europeans. Differences in linkage disequilibrium distribution between populations may have contributed to the discrepancy (Hara et al., 2014). Strictly, MCT13 first appeared in another study: the SLC16A11 risk haplotype identifying GWAS published in the same year described an association at chromosome $17 \mathrm{p} 13.1$ spanning the two adjacent located genes $S L C 16 A 11$ and $S L C 16 A 13$, but localized the strongest association signal to the SLC16A11 gene (Williams et al., 2014). Therefore, both GWAS highlight a potential role of MCT13 in the development of T2D. Yet, the function and biology of MCT13 are unknown. MCT13 is located at the membrane of the Golgi apparatus (Fig. 1) and highly expressed in the liver, kidney, and intestine (Fig. 2) (Williams et al., 2014). Compared with MCT11, GWAS highlighting a potential role of MCT13 in metabolic disease are limited, and substrate(s) and physiological function of the protein are not characterized at all (Table 1). One mouse study showed an upregulation of Slc16a13 gene expression in the small intestine after treatment with a PPAR $\alpha$ agonist (Hirai et al., 2007). Because PPAR $\alpha$ is a key regulator of hepatic lipid metabolism, these mouse data are in line with the notion that MCT13 regulates lipid metabolism and that MCT13 deregulation may contribute to lipid-induced insulin resistance and T2D.

Finally, functional studies are necessary to understand if and how the MCT13 transporter could be modulated to counteract T2D development. Whether 
activation, as proposed for MCT11, or inhibition could improve metabolic disease is unknown. Because the SLC16A13 gene has been directly linked to T2D in humans, the transporter should be investigated further. 4. Therapeutic Potential of Solute Carrier 16 Transporters. Various studies highlight the role of SLC16 family members in physiology and pathophysiology. The largest data sets exist for the best-characterized transporters SLC16A1/MCT1, SLC16A3/MCT4, and SLC16A7/MCT2. However, few genetic studies have linked these monocarboxylate transporters to metabolic disease. More detailed knowledge concerning expression pattern, substrate specificity, and function in health and disease as well as biochemical and molecular mechanisms is required to judge whether SLC16 family members are potential drug targets for metabolic disease. MCT1 is primarily discussed as a target in cancer therapy, and human genetic data linking $S L C 16 A 1$ genetic variants to metabolic disease are scarce, although mouse data are convincing. SLC16A13 has to date only been identified in one GWAS. Additional genetic studies and a valid mouse model are needed to verify the link between MCT13 function and T2D. SLC16A11 has been directly linked to human T2D in substantial GWAS, and the MCT11 transporter has also partially been functionally characterized in disease models (Table 1). Currently, MCT11 is the most attractive candidate to target for the treatment of metabolic disease. Additional SLC16 transporters such as SLC16A3/ MCT4, SLC16A9/MCT9, and SLC16A10/MCT10 have been linked to diabetic nephropathy, gout, and NAFLD, respectively (Table 2). Detailed information of therapeutic potential of these SLC16 family members is provided in Emerging Potential of Other Solute Carrier Target Structures.

\section{Solute Carrier 25: The Mitochondrial Carrier Family}

The SLC25 gene family is the largest of the SLC transporter superfamily, comprising 53 members with highly diverse substrate specificities and tissue expressions. SLC25 genes encode mitochondrial carriers that are mostly localized in the inner mitochondrial membrane (Fig. 1). Their substrates vary widely in structure and size from small ions like $\mathrm{H}^{+}$to the larger molecules such as ATP or coenzyme A (Palmieri, 2004). For many SLC25 family members, substrate specificities remain unknown (Palmieri, 2013). Based on the substrates transported, mitochondrial carriers can be classified as carriers for amino acids, carboxylates, keto acids, nucleotides, dinucleotides, coenzymes, and other substrates. Carriers of the SLC25 family are further divided into subfamilies, which are characterized by distinctive structural features, substrate specificity, mode of transport, and kinetic properties. Functionally, SLC25 transporters have been implicated in diverse metabolic pathways, including oxidative phosphorylation,
TCA cycle, fatty acid oxidation, gluconeogenesis, lipogenesis, ketone body production and utilization, urea synthesis, amino acid degradation, regulation of nucleotide and deoxynucleotide pools in the mitochondrial matrix, heat production, and many more (Palmieri, 2004, 2013; Palmieri and Monne, 2016). The SLC25 family is associated with human diseases, highlighting the essential function of solute transport through the inner mitochondrial membrane in linking mitochondrial and cytosolic metabolism (Palmieri, 2004, 2013; Palmieri and Monne, 2016).

A subfamily of mitochondrial carriers includes the uncoupling proteins (UCPs) that play an important role in energy homeostasis. It consists of six members, of which UCP1 (SLC25A7), UCP2 (SLC25A48), and UCP3 (SLC25A9) are the most prominent. Generally, substrate oxidation is coupled to ATP formation. Oxidative phosphorylation generates a proton gradient by the respiratory chain across the inner mitochondrial membrane, which is used to generate ATP from ADP through flowback of the protons mediated by ATPase. UCPs can uncouple ATP production from mitochondrial respiration, thereby dissipating energy as heat (Schrauwen et al., 1999a). UCP1, UCP2, and UCP3 are mitochondrial proton transporters (Fig. 1) that are linked to obesity and T2D in both human studies and rodent models (Schrauwen et al., 1999a; Dalgaard and Pedersen, 2001; Hesselink et al., 2003; Langin, 2003; Jia et al., 2009; Dalgaard, 2011; Souza et al., 2011; Brondani et al., 2012; Liu et al., 2013; Busiello et al., 2015; Giralt and Villarroya, 2017; Ost et al., 2017; Sreedhar and Zhao, 2017) (Table 1).

\section{Solute Carrier 25A7/Uncoupling Protein 1.}

a. Mitochondrial uncoupling and thermogenesis. UCP1, also known as thermogenin, is encoded by the $S L C 25 A 7$ gene and is expressed in brown adipose tissue (Fig. 2). In contrast to white adipose tissue, which is the primary site of energy storage, brown adipose tissue contains large amounts of mitochondria. Through uncoupled mitochondrial respiration, brown adipose tissue regulates thermogenesis. UCP1 is responsible for the mitochondrial proton leak (Nicholls et al., 1978) and is the UCP, which mediates energy dissipation as heat (Golozoubova et al., 2001; Nedergaard et al., 2001). The term "mitochondrial proton leak" defines a nonATP-consuming process that takes place when inner mitochondrial membrane proton conductance is increased and the generated proton gradient is insufficient such that oxygen consumption is uncoupled from ATP production. Because mitochondria of brown adipose tissue are particularly permeable to protons, proton leakage in this tissue directly dissipates energy as heat, explaining the thermogenic activity of brown adipose tissue (Nicholls and Locke, 1984). Brown adipose tissue thermogenesis plays a key role in the development of obesity in animal models (Rothwell and Stock, 1979; Lowell et al., 1993; Feldmann et al., 2009; 
Kajimura and Saito, 2014) and in humans (Nedergaard et al., 2007; Cypess et al., 2009; Saito et al., 2009; van Marken Lichtenbelt et al., 2009; Kiefer, 2017; Leitner et al., 2017).

b. Solute carrier $25 A 7$ genetic variants. Numerous human studies have linked SLC25A7 polymorphisms to obesity and T2D (Table 1). Most association studies focused on the rs1800592 polymorphism in the $S L C 25 A 7$ promotor region that has been associated with reduced SLC25A7 mRNA expression in obese subjects (Esterbauer et al., 1998). The datasuggest that reduced protein function increases susceptibility to metabolic disease. However, findings across more than 30 human genetic studies, including subjects from different populations worldwide, varied. Some studies showed associations between one or more $S L C 25 A 7$ polymorphisms with obesity, diabetes, or other metabolic syndrome characteristics. Others did not show associations between $S L C 25 A 7$ polymorphisms and metabolic traits (Brondani et al., 2012). Data remain conflicting regarding the effect of the allelic variants. For example, whereas the G-allele of the rs1800592 polymorphism $(-3826 \mathrm{~A}>\mathrm{G})$ was significantly associated with increased BMI (Chathoth et al., 2018), another study reported that G-allele carriers had lower body weight and fat mass and homozygous G-allele carriers had lower frequency of T2D compared with patients carrying the A-allele (Nicoletti et al., 2016). Other studies did not detect any association between the SLC25A7 polymorphism and BMI, obesity, or T2D risk (de Souza et al., 2013; Brondani et al., 2014a,b).

c. Metabolic phenotype of animal models. The idea that UCP1 dysfunction promotes obesity has been extensively investigated in mouse models. As early as 1978, defects in thermogenesis were demonstrated in genetically obese $o b / o b$ mice that were cold-sensitive (Himms-Hagen and Desautels, 1978). Transgenic mice with the primary deficiency of brown adipose tissue lacking UCP1 became obese at a young age in the absence of hyperphagia. This finding indicates that brown adipose tissue-deficient mice have increased metabolic efficiency (Lowell et al., 1993). However, Slc25a 7 knockout mice were cold-sensitive, indicating defective thermoregulation, but the animals did not become obese on either a standard diet or HFD. Loss of UCP1 could therefore be compensated in part by UCP2 that is induced in brown fat of UCP1-deficient mice. Other signals that are involved in the regulation of body weight could also contribute to the finding (Enerbäck et al., 1997).

Transgenic mice with $S l c 25 a 7$ expression in white adipose tissue exhibit markedly reduced HFD-induced obesity in association with a comparable total oxidative capacity in white and brown adipose tissue (Kopecky et al., 1995; Kopecký et al., 1996a,b; Baumruk et al., 1999). Ectopic Slc25a7 expression in epididymal fat through adenovirus vector injection improves glucose tolerance and decreases food intake in both diet-induced and genetically obese mouse models (Yamada et al., 2006). Transgenic Slc25a 7 expression in mouse skeletal muscle increased muscle oxygen consumption dramatically and protected from diet-induced obesity, improved insulin sensitivity, and increased metabolic rate at rest and with exercise ( $\mathrm{Li}$ et al., 2000). In a genetic obese mouse model, tetracycline-inducible Slc25a7 expression in skeletal muscle lowered body weight, increased oxygen consumption, improved glucose tolerance, and unexpectedly lowered blood pressure (Gates et al., 2007).

d. Mitochondrial uncoupling to treat metabolic disease. The so-called browning of white adipose tissue has received much attention in recent years. Today, it is known that UCP1 can be induced in white fat cells by cold as well as pharmacological, nutritional, and endogenous stimuli (Ost et al., 2017). Although the presence of brown fat depots in adult humans was questioned, SLC25A7-expressing adipocytes in adults were shown in multiple studies and also associated with lower mass and/or activity in obese and older subjects (Cypess et al., 2009; Saito et al., 2009; van Marken Lichtenbelt et al., 2009; Virtanen et al., 2009; Leitner et al., 2017).

Obesity treatment aims to reduce energy intake and/ or increase energy expenditure. Therapeutic intervention reduces energy intake either by affecting satiety centers in the brain such as sibutramine (Bray et al., 1999; James et al., 2000; Ryan, 2000) and glucagon-like peptide 1 receptor agonists (O'Neil et al., 2018), or by decreasing the efficiency of intestinal absorption such as orlistat (Sjöström et al., 1998). Chemical agents such as 2,4-dinitrophenol or carbonyl cyanide p-(trifluoromethoxy) phenylhydrazone increase energy expenditure by uncoupling mitochondrial oxidative respiration. However, life-threatening side effects limit the use of such compounds (Parascandola, 1974). More recently, liver-specific mitochondrial protonophores, called controlled-release mitochondrial protonophores, produce mild hepatic mitochondrial uncoupling, ameliorated hepatic steatosis, and insulin resistance in a HFD-fed rat model and steatohepatitis in a rat model of nonalcoholic steatohepatitis (Perry et al., 2015). The treatment also prevented hepatic steatosis, insulin resistance, and T2D in lipodystrophic mice (Abulizi et al., 2017). Given this proof of concept, mitochondrial uncoupling targeting UCP1 received renewed interest to modulate whole-body energy homeostasis and to treat obesity and related metabolic diseases (Ost et al., 2017).

Taken together, mouse and human studies indicate an essential role of UCP1 in body weight regulation, T2D, and arterial hypertension. Therefore, modulation of brown adipose tissue thermogenesis to increase energy expenditure could be an effective strategy against obesity. Reduced SLC25A7 gene expression in obese subjects indicates reduced transporter activity as causative for the related disease phenotypes, and mouse 
models clearly support the idea of protection from dietinduced obesity due to ectopic Slc25a7 expression. Most importantly, a very recent publication identified the chemical compound linifanib as a potent inducer of UCP1 expression in primary inguinal adipocytes in vitro and in vivo (Zhao et al., 2019a). Of note, linifanib (ABT-869), an orally bioavailable, small-molecule receptor tyrosine kinase inhibitor, has been reported to exhibit potent antiangiogenic and antitumor effects in preclinical studies and is currently undergoing phase III trials (McKeegan et al., 2015; Horinouchi, 2016). Thus, additional research on the effect of a specific UCP1 activator in the context of obesity seems to be enormously promising, and existing linifanib preclinical studies could facilitate its application in obese subjects. In contrast to existing anti-obesity drugs, an UCP1 activator might have additional metabolic benefits regarding diabetes and hypertension. However, the safety profile will need to be carefully studied due to the possibility of hyperthermia, and the parallel effect of receptor tyrosine kinase inhibition in the patients must be critically investigated.

2. Solute Carrier 25A8/Uncoupling Protein 2 and Solute Carrier 25A9/Uncoupling Protein 3.

a. Physiological functions irrespective of mitochondrial uncoupling. UCP2, encoded by SLC25A8, was identified in 1997 as novel mitochondrial uncoupling protein that, unlike UCP1, is widely expressed in human tissues (Fleury et al., 1997; Gimeno et al., 1997) (Fig. 2). UCP3, product of the SLC25A9 gene predominantly expressed in skeletal muscle, was identified shortly thereafter (Boss et al., 1997; Lowell, 1999) (Fig. 2). Because the two genes are localized adjacent on chromosome 11, they may result from an ancestral gene duplication. In contrast to UCP1, the physiological functions of UCP2 and UCP3 are less well defined and may include the export of fatty acids or fatty derivates from the mitochondrial matrix (Moore et al., 2001; Goglia and Skulachev, 2003; Schrauwen et al., 2003; Schrauwen and Hesselink, 2004; Bouillaud et al., 2016). Other physiological functions involve antioxidant mechanisms, which may maintain the balance between reactive oxygen species production and clearance. A possible side effect of proton leaks is a higher flow rate through the electron-transport chain, and therefore a decrease in the half-life of radical intermediates, which can donate its electron to oxygen and create reactive oxygen species (Dalgaard and Pedersen, 2001). UCP2 and UCP3 might dissipate the proton gradient to prevent reactive oxygen species generation, thereby regulating oxidative stress and cell signaling (Echtay et al., 2002; Mailloux and Harper, 2011; Bouillaud et al., 2016).

b. Solute carrier $25 A 8$ and solute carrier $25 A 9$ genetic variants. The first SLC25A8 and SLC25A9 gene polymorphisms were identified in Danish persons with obesity (Urhammer et al., 1997, 1998). In the last two decades, allelic variants of the genes have been studied in different populations (Table 1). Dalgaard and Pedersen (2001) and Dalgaard (2011) reviewed physiologic al functions of both transporters and known polymorphisms that are associated with obesity and T2D. Three common SLC25A8 polymorphisms are well studied in humans: the promoter variant rs659366 $(-866 \mathrm{G}>\mathrm{A})$, the missense variant $r s 660339$ (c. $164 \mathrm{C}>\mathrm{T}$; p.A55V), and a 45-bp insertion-deletion polymorphism in the $3^{\prime}$ untranslated region. The rs659366 promoter minor A-allele most likely directs higher rates of transcription from the SLC25A8 promoter compared with the G-allele (Dalgaard, 2011). Conflicting studies show the polymorphism to be associated with a reduced risk of obesity (Esterbauer et al., 2001; Jun et al., 2009), but also with increased obesity prevalence (Dhamrait et al., 2004; Ochoa et al., 2007; Kring et al., 2008). Several studies revealed no associations, suggesting that UCP2 has only a modest effect, if any, on the development of obesity (Dalgaard, 2011). Similarly, human data on $\beta$-cell function and T2D are partially conflicting. Heterozygous SLC25A8 mutations were reported in patients suffering from congenital hyperinsulinemia (Gonzalez-Barroso et al., 2008). Because SLC25A8 null mutations are related to hyperinsulinemia, the A-allele of the rs659366 variant, resulting in increased gene expression, is expected to show association with decreased $\beta$-cell function and ultimately with T2D (Dalgaard, 2011). Indeed, decreased glucosestimulated insulin secretion was observed in two studies (Sesti et al., 2003; Sasahara et al., 2004), and decreased basal insulin secretion was reported among A-allele carriers (Esterbauer et al., 2001). Others did not reproduce the associations (Dalgaard et al., 2003; Sesti et al., 2003; D'Adamo et al., 2004; Ji et al., 2004; Bulotta et al., 2005). Moreover, both alleles of the rs659366 variant were associated with T2D (Sasahara et al., 2004; Lyssenko et al., 2005; Gable et al., 2006). Both increased risk of hypertension (Ji et al., 2004) as well as decreased risk of coronary artery disease in subjects with T2D (Cheurfa et al., 2008; Palmer et al., 2009) have been reported to be associated with the A-allele of the rs659366 polymorphism. Given the conflicting data, larger samples with well-characterized participants are required to detect the true effect of an identified variant (Dalgaard, 2011). A more recent metaanalysis of 42 studies evaluated associations of the three common SLC25A8 gene polymorphisms with overweight and obesity: the T-allele of $r s 660339$ polymorphism was associated with an increased risk of obesity, the A-allele of the rs659366 promotor polymorphism protected from obesity, and the 45-bp insertion/deletion polymorphism was not associated with obesity susceptibility (Zhang et al., 2014).

The promoter variant $r s 1800849(-55 \mathrm{C}>\mathrm{T})$ of the SLC25A9 gene is common and well-studied (Cassell et al., 2000; Dalgaard et al., 2001a,b; Berentzen et al., 
2005). The T-allele results in increased SLC25A9 expression in skeletal muscle and may raise resting energy expenditure (Schrauwen et al., 1999c; Kimm et al., 2002). Low resting energy expenditure promotes weight gain. Indeed, one study revealed a negative correlation between SLC25A9 expression and BMI (Schrauwen et al., 1999b). Furthermore, the T-allele was associated with reduced BMI, increased highdensity lipoprotein-cholesterol levels (Schrauwen et al., 1999c; Liu et al., 2005; Hamada et al., 2008), reduced T2D risk, and higher plasma total cholesterol and low-density lipoprotein cholesterol concentrations (Meirhaeghe et al., 2000). However, multiple studies did not observe associations between the rs1800849 polymorphism and metabolic rate, obesity, BMI, insulin secretion, or T2D (Walder et al., 1998; Dalgaard et al., 2003; Berentzen et al., 2005; Ochoa et al., 2007; Hsu et al., 2008). Some showed a reverse association of the T-allele with higher BMI and waist circumference (Otabe et al., 2000; Herrmann et al., 2003; Lindholm et al., 2004). Jia et al. (2009) provided a comprehensive overview of SLC25A9 polymorphisms and noticed the discrepancies between studies that might be explained partially by influences of adjacent loci in the same haplotype, as well as the exclusion of key lifestyle factors in the statistical analyses.

The potential role of both genes in metabolic disease is further supported by studies indicating that expression of SLC25A8 and SLC25A9 predicts diabetes onset in humans. Variation in the SLC25A8-SLC25A9 gene cluster is associated with an increased T2D risk (Gable et al., 2006). Moreover, reduced UCP3 protein levels were reported in individuals with prediabetes and T2D (Schrauwen et al., 2006). Recent meta-analyses detected significant associations between the $S L C 25 A 8$ and SLC25A9 polymorphisms and BMI, obesity, and T2D (de Souza et al., 2013; Brondani et al., 2014a,b). However, associations markedly differed between different populations; a recent cross-sectional study of European adolescents failed to identify any association between SLC25A8 or SLC25A9 variants and adiposity, whereas the C-allele of the SLC25A7 rs6536991 intron variant was associated with a lower risk of overweight (Pascual-Gamarra et al., 2019). Mechanisms and phenotypes are not clear to date, and, as for every GWAS, interindividual differences, including ethnic, lifestyle, environmental and genomic factors, and nutritional characteristics, result in different effects of described SLC25A8 and SLC25A9 polymorphisms on obesity and related phenotypes. These factors could interfere with UCP2 and UCP3 physiology and pathophysiology. Regarding limited consistency between studies, further human studies and functional characterization of both transporters could help to solve the unanswered questions.

c. Metabolic phenotype of animal models. Mouse studies support links between UCP2 and UCP3 and obesity and related disorders, but also present varying phenotypes. Increased Slc25a $8 \mathrm{mRNA}$ levels have been observed in obesity-resistant A/J mice compared with obesity-prone B6 mice. Moreover, HFD induced Slc25a8 in white adipose tissue. The findings suggest that SLC25A8 might play a role in the development of hyperinsulinemia and obesity (Fleury et al., 1997). UCP2 does not seem to regulate body weight or coldinduced thermogenesis in mice (Arsenijevic et al., 2000; Zhang et al., 2001). UCP2-deficient mice exhibited increases in insulin and lower blood glucose levels. Furthermore, pancreatic islets lacking UCP2 feature increased ATP levels and increased insulin secretion. The finding indicates that UCP2 negatively regulates glucose-stimulated insulin secretion possibly linking obesity and $\beta$-cell dysfunction. Indeed, UCP2 is upregulated in obese $o b / o b$ mice, and UCP2 deficiency markedly improved their diabetic phenotype (Zhang et al., 2001). However, contrary to results in UCP2-deficient mice of mixed 129/B6 strain background (Zhang et al., 2001), glucose-stimulated insulin secretion in UCP2deficient islets of congenic mouse strains was significantly decreased, and these mice also did not become diabetic ( $\mathrm{Pi}$ et al., 2009). Increased oxidative stress in UCP2-deficient mice may contribute to declining $\beta$-cell function, but overall, the pathophysiological role of UCP2 is unclear.

UCP3 deficiency in mice does not affect body weight regulation, exercise tolerance, fatty acid oxidation, or cold-induced thermogenesis. Yet, reactive oxygen species are more abundant in skeletal muscle, indicating that UCP3 protects from oxidative stress (Gong et al., 2000; Vidal-Puig et al., 2000). Transgenic mice overexpressing human SLC25A9 in skeletal muscle are hyperphagic. Nevertheless, body weight and adipose tissue mass are reduced, together with lower fasting plasma glucose and insulin levels and an increased glucose clearance rate (Clapham et al., 2000; Horvath et al., 2003). Strikingly, UCP2 and UCP3 deficiency in mice is associated with increased production of reactive oxygen species that is known to be involved in insulin resistance (Houstis et al., 2006). Therefore, modulation of UCP2 and UCP3 activity may protect from insulin resistance in addition to obesity.

Finally, although SLC25A8 and SLC25A9 polymorphism may be associated with obesity, diabetes, or related phenotypes, their impact on these phenotypes seems to be modest with large heterogeneity between studies (Dalgaard and Pedersen, 2001). To date, the effects of UCP2 and UCP3 modulation remain elusive because human and mouse data did not clearly demonstrate whether the transporters should be activated or inhibited for therapeutic intervention. The functional characterization based on human association studies and on mouse models is very complex and needs further evaluation. Nevertheless, the link between UCP 
function and dysfunction and metabolic disease is well established and supports the idea of targeting UCPs.

3. Solute Carrier 25A13/Aspartate-Glutamate Carrier 2.

a. Mitochondrial aspartate-glutamate transport. The aspartate-glutamate carrier 2 (AGC2), also known as citrin, adult-onset citrullinemia type II (CTLN2), or aralar2, is encoded by the $S L C 25 A 13$ gene and facilitates the calcium-dependent exchange of cytoplasmic glutamate with mitochondrial aspartate across the inner mitochondrial membrane (Fig. 1). AGC2 is a key component of the malate-aspartate shuttle and essential to export aspartate from the mitochondrial matrix to the cytosol. Aspartate is required for urea synthesis from ammonia and alanine, protein synthesis, gluconeogenesis from lactate, as well as the oxidation of cytosolic NADH (Palmieri, 2004). The transporter is the only aspartate-glutamate carrier isoform expressed in the liver (Fig. 2). Hence, in the liver, mutations in the SLC25A13 gene cannot be compensated by another transporter system and can cause two age-dependent autosomal-recessive diseases: neonatal intrahepatic cholestasis by citrin deficiency (NICCD) and CTLN2, an accumulation of ammonia and other toxic substances in the blood.

b. Solute carrier $25 A 13$ genetic variants. Mutated SLC25A13 has been identified in patients suffering from type II citrullinemia and proposed to affect urea cycle function (Kobayashi et al., 1999). In 2001 , two reports linked SLC25A13 mutations to a neonatal liver disease (Ohura et al., 2001; Tazawa et al., 2001). Multiple case reports with over 50 different SLC25A13 gene mutations further underpin AGC2 dysfunction in human metabolic disease (Table 1). Hepatocytes have limited capacity to take up extracellular aspartate. Therefore, both AGC2-related disease phenotypes are mainly caused by reduced cytosolic aspartate and hepatic NADH reoxidation (Palmieri and Monne, 2016). AGC2 deficiency decreases hepatic argininosuccinate synthetase activity, causing hyperammonemia and citrullinemia. Additionally, reduced malate-aspartate shuttle activity increases the cytosolic $\mathrm{NADH} / \mathrm{NAD}^{+}$ratio, resulting in the inhibition of glycolysis and alcohol metabolism. The mechanisms explain carbohydrate and alcohol intolerance in affected patients, which exacerbates symptoms, and preference for high-protein diets containing aspartate. Highcarbohydrate diets increase hepatic triglycerides in some patients, likely due to compensatory malatecitrate shuttling transferring reducing equivalents to the mitochondria, thereby producing acetyl-CoA in the cytosol and stimulating fatty acid synthesis (Palmieri, 2004).

Neonatal NICCD is characterized by transient intrahepatic cholestasis, fatty liver, hepatomegaly, ketotic hypoglycemia, hypoproteinemia, and aminoacidemia. Due to liver dysfunction, some patients experience hepatitis, jaundice, decreased coagulation factors, hemolytic anemia, and bleeding diathesis. NICCD is usually benign, and symptoms disappear after a few years. In adulthood, some patients develop CTLN2, which is associated with hypoproteinemia and hyperammonemia, leading to a neurological manifestation, including encephalopathy and neuropsychiatric symptoms (Palmieri, 2004, 2013; Palmieri and Monne, 2016). Some adult patients also manifest fatty liver and hyperlipidemia unrelated to obesity or ethanol consumption, pointing to the essential function of the aspartate-glutamate transporter in hepatic metabolism. Finally, SLC25A13 mutations have been linked to steatosis, nonalcoholic steatohepatitis, and hepatocellular carcinoma (Takagi et al., 2006; Tsai et al., 2006; Fukumoto et al., 2008; Komatsu et al., 2008; Chang et al., 2011). For patients with CTLN2, liver transplantation is one of the most promising prospects. However, dietary supplementation can ameliorate symptoms; patients with NICCD usually respond to medium-chain triglyceride supplements and lactoserestricted formulas, whereas medium-chain triglyceride supplements with low-carbohydrate formulas are recommended for CTLN2 (Hayasaka et al., 2012, 2014, 2018; Hayasaka and Numakura, 2018).

c. Metabolic phenotype of animal models. Beneficial responses to dietary interventions have also been shown in Slc25a13 knockout mice, indicated through increased food intake and maintained body weight following supplementation with protein or medium-chain triglycerides (Saheki et al., 2012). Initially, the Slc25a13 knockout mouse model failed to mimic CTLN2 and did not show changes in glucose, amino acid, or ammonia metabolism. At least in mice, AGC2 deficiency may not be sufficient to elicit liver disease (Sinasac et al., 2004). Because enhanced glycerol phosphate shuttle activity may be compensating for the loss of AGC2 function, Slc25a13 Gpd2 (glycerol 3-phosphate dehydrogenase 2) double-knockout mice were generated. The model developed citrullinemia, hyperammonemia, hypoglycemia, and a fatty liver, displaying features of human AGC2 deficiency (Saheki et al., 2007).

Taken together, the phenotypes of AGC2 deficiency have been extensively investigated in human beings and in mice (Saheki and Song, 2005; Hayasaka and Numakura, 2018). However, AGC2 was never suggested as target to treat fatty liver, possibly because dietary interventions and liver transplantation are well established treatments for NICCD and CTLN2. Moreover, pharmacological activation of the transporter seems to be challenging. AGC2 deficiency associated with fatty liver is only one symptomatic feature in humans and has never been directly related to obesity or metabolic disease. Given the complex physiological function of the hepatic aspartate-glutamate carrier, additional research on the role of AGC2 in obesity-related metabolic disease 
may be worthwhile and could generate novel therapeutic concepts beyond the two well-known human phenotypes.

\section{Solute Carrier 25A20/Carnitine-Acylcarnitine Carrier.}

a. Mitochondrial carnitine-acylcarnitine transport. The broadly expressed mitochondrial carnitineacylcarnitine carrier (CAC), product of the SLC25A20 gene, catalyzes the exchange between cytosolic acylcarnitine that enters the mitochondria and intramitochondrialfree carnitine that is transferred into the cytosol (Figs. 1 and 2). Thereby, CAC is a key component of the carnitine shuttle system. The system transfers acyl groups from acyl-CoA to carnitine by the outer mitochondrial membrane carnitine palmitoyltransferase 1 , translocates acylcarnitines across the inner mitochondrial membrane by CAC, and finally transfers the acyl groups from acylcarnitines to $\mathrm{CoA}$ inside mitochondria by carnitine palmitoyltransferase 2 . The mechanism imports acyl groups of cytosolic long-chain fatty acids into mitochondria. In mitochondria, acyl groups are oxidized by the $\beta$-oxidation pathway, the major energy source for heart and skeletal muscle during periods of fasting and physical exercise (Indiveri et al., 2011; Palmieri, 2013). As this pathway is fundamental in cellular energy metabolism, CAC transporter dysfunction leads to a severe disease known as CAC deficiency (Table 1).

b. Solute carrier $25 A 20$ genetic variants. CAC deficiency was described in 1992 as an autosomal recessive disorder (Stanley et al., 1992). The first pathogenic SLC25A20 gene mutation was reported in 1997 (Huizing et al., 1997). Over 40 additional mutations have since been described as resulting in the complex disease pattern that clinically manifests with episodes of coma upon fasting, cardiomyopathy, hypotonia, muscle weakness, respiratory distress, seizures, and hepatic dysfunction (Indiveri et al., 2011; Palmieri and Monne, 2016). Multiple circulating metabolites are abnormal in this condition. Key findings include hypoglycemia secondary to oxidation of glucose instead of ketone bodies in the muscle during fasting, as well as hypoketosis, hyperammonemia, dicarboxylic aciduria, and increased levels of transaminases and long-chain acylcarnitines. The clinical symptoms are well characterized and based on the energy deficit in tissues that rely on fatty acid oxidation. Moreover, elevated acylcarnitine levels may damage the heart and skeletal muscle, whereas hypoketosis, hyperammonemia, and hypoglycemia result in the neurological phenotype (Indiveri et al., 2011; Palmieri, 2013). The disease can occur in a mild or severe form, with remaining protein activity of about $5 \%$ or below $1 \%$, respectively. CAC pathophysiology, common clinical features, and interventions of the disease have been reviewed recently (Indiveri et al., 2011; Yan et al., 2017). The severe phenotype is usually present in the neonatal period with a high mortality rate within the first year of life due to cardiac arrest and respiratory failure (Yan et al., 2017). Therefore, early appropriate diagnosis and treatment are necessary to manage the highly lethal disorder and to ameliorate disease progression. Therapeutic approaches include intravenous glucose injection to suppress fatty acid mobilization and oxidation, as well as ammonia detoxification through arginine administration. Longterm management requires strict diet with frequent meals to avoid fasting periods, high-carbohydrate intake, medium-chain triglyceride supplementation, and limitation of long-chain fatty acids (Indiveri et al., 2011; Yan et al., 2017).

In summary, given the severity of CAC deficiency and the limited success of existing therapies, research on new therapeutic approaches is needed. Moreover, whether direct CAC modulation could ameliorate other disorders, including obesity-related metabolic disease, deserves to be studied. However, more common genetic polymorphisms may not result in a dramatic loss of protein activity and have not been associated with other disease phenotypes. Slc25a20 knockout mice are not available to test its obesity-related phenotype under HFD feeding. Keeping in mind the crucial function of the transporter in energy metabolism and the severe metabolic defects in CAC deficiency, research in this direction should be considered.

\section{Solute Carrier 25A24 / ATP-Mg / Phosphate Carrier} Protein 1.

a. Mitochondrial transport of adenine nucleotides. The mitochondrial ATP-Mg/phosphate carrier protein (APC)1, encoded by SLC25A24, is broadly expressed and facilitates exchange of adenine nucleotides, including ATP-Mg, ATP, ADP, and AMP, and phosphate between mitochondrial matrix and cytosol (Figs. 1 and 2). The transporter regulates adenine nucleotide concentrations in the mitochondrial matrix, thereby affecting mitochondrial adenine-nucleotide-dependent enzymes, which regulate gluconeogenesis from lactate, urea synthesis, mitochondrial DNA replication, transcription, and protein synthesis, among others (Palmieri and Monne, 2016).

b. Solute carrier 25A24 genetic variants. SLC25A24 was proposed as a novel susceptibility gene for low fat mass in humans (Urano et al., 2015) (Table 1). Using SNP arrays, three SNPs in the SLC25A24 gene were associated with body fat percentage and BMI ( $r s 491785, r s 519129$, and rs547364) in Japanese postmenopausal women (Urano et al., 2015). Subjects harboring the major alleles had lower body fat percentage and BMI and the rs491785 major allele associated with lower SLC25A24 mRNA expression in human preadipocytes. No additional gene variants linked to disease phenotypes in other populations and/ or larger study cohorts are known.

c. Metabolic phenotype of animal models. Slc25a24 knockout mice are resistant to HFD-induced obesity with significantly reduced white adipose tissue 
and liver weights (Urano et al., 2015). The phenotype was linked to reduced adipocyte differentiation marker expression and reduced hepatic triglycerides in Slc25a24 knockout mice (Urano et al., 2015).

In conclusion, APC1 may be linked to obesity in humans and mice. Further studies are needed to verify these data and clarify APC1-regulated mechanisms and (patho)physiology in more detail.

6. Therapeutic Potential of Solute Carrier 25 Transporters. Summarizing the SLC25 family of mitochondrial carriers (Table 1), SLC25A7/UCP1 seems to be the most promising candidate to target for the treatment of obesity and related metabolic diseases. Although SLC25A8/UCP2 and SLC25A9/UCP3 are linked to obesity, diabetes, or related phenotypes, studies are heterogeneous and partially controversial. Nevertheless, the link between UCP function and dysfunction and metabolic disease is well established, especially for UCP1, and the obvious therapeutic potential should be pursued. In addition to these potential targets presented in this section, two additional transporters, SLC25A25/APC3, which was linked to resistance to diet-induced obesity in a mouse model, and SLC25A40/mitochondrial carrier family protein (MCFP), which was associated with hypertriglyceridemia in one human study (Table 2), are discussed in Emerging Potential of Other Solute Carrier Target Structures.

\section{Solute Carrier 30: The Zinc Transporter Family}

Zinc is crucial for the function of numerous enzymes and proteins that participate in the metabolism of proteins, nucleic acids, carbohydrates, and lipids (Vallee and Falchuk, 1993). It affects various biological processes, including growth, development, and neuronal and immune function, and its cellular homeostasis needs to be tightly controlled as both zinc deficiency and excess are toxic to cells (Sekler et al., 2007). Zinc levels are regulated by metalloproteins and two different families of zinc transporters with opposite functions: SLC30 and SLC39. Transporters of the SLC30 family [zinc transporter (ZnT)] mediate efflux from the cytosol into cytosolic compartments or the extracellular space when cytosolic zinc concentration is high. Transporters of the SLC39 family (zinc-iron permease) mediate zinc influx into the cytoplasm in the face of low cellular concentration (Palmiter and Huang, 2004). Although ZnT1 (SLC30A1) primarily functions as plasma membrane efflux transporter, other family members are mainly localized in intracellular membranes. The SLC30 family consists of 10 members that are expressed at the plasma membrane to transport cytoplasmic zinc out of the cell, thereby preventing zinc toxicity, or at intracellular membranes to shuttle cytoplasmic zinc into cellular compartments for secretion, storage, or supply for zinc-containing proteins (Palmiter and Huang, 2004; Huang and Tepaamorndech,
2013). The detrimental effects of altered zinc transporter activity and dysregulation of intra- and extracellular zinc concentrations are illustrated by genetic studies in humans and in mice. Pathology resulting from altered SLC39 transporter function includes abnormal embryonic and immune cell development. In contrast, SLC30 proteins are associated with diabetes, Alzheimer's, or Parkinson disease (Hara et al., 2017).

\section{Solute Carrier 30A8/Zinc Transporter 8.}

a. Zinc and insulin biosynthesis. The most prominent member of the SLC30 family in terms of metabolic disease is $\mathrm{ZnT}$, product of the SLC30A8 gene. The transporter is mainly expressed in the membrane of insulin secretory granules in pancreatic $\beta$-cells (Figs. 1 and 2) and provides zinc for insulin synthesis (Chimienti et al., 2004). Zinc concentrations are particularly high in $\beta$-cells, especially in insulin secretory granules, where it forms crystalloid-like hexamers with insulin. The process maintains insulin maturation, storage, and stability (Emdin et al., 1980; Hutton, 1989; Foster et al., 1993; Dodson and Steiner, 1998). The critical role of zinc in insulin biosynthesis was first proclaimed when Scott and Fisher (1938) observed concomitant reductions in insulin and zinc in the pancreas, but unaltered zinc concentrations in the livers of patients with diabetes. Serum zinc concentrations are also significantly reduced in patients with diabetes (Garg et al., 1994). The complex relationship between zinc and both type 1 and type 2 diabetes has further been established (Chausmer, 1998; Maret, 2017; Fukunaka and Fujitani, 2018). Zinc deficiency in patients with diabetes led to the idea of zinc supplementation to promote $\beta$-cell function. The intervention showed beneficial metabolic effects in humans and in T2D animal models (Taylor, 2005; Jayawardena et al., 2012).

b. Solute carrier 3048 genetic variants. Whether zinc deficiency in patients with diabetes is the cause or consequence of the disease was not clear for a long time. However, a potentially causal relationship was discovered with the identification of a $S L C 30 A 8$ sequence variant that increased T2D susceptibility in various populations worldwide (Table 1). Furthermore, the transporter was identified as a major autoantigen in human type 1 diabetes (Wenzlau et al., 2007). The link to T2D arose with the identification of the SLC30A8 SNP rs13266634 as risk loci for T2D in three independent GWAS (Saxena et al., 2007; Scott et al., 2007; Sladek et al., 2007). The first study analyzed polymorphisms in French T2D patients and identified four novel loci, including SLC30A8. Two additional studies in Finnish and Swedish subjects described the same SLC30A8 sequence variant (Saxena et al., 2007; Scott et al., 2007). The rs13266634 polymorphism leads to a single base change from $\mathrm{C}$ to $\mathrm{T}$ with consequent amino acid change from arginine (R) to tryptophan (W) at position 325 (c.973C $>$ T; p.R325W). Increased T2D risk 
was associated with the major C-allele and accordingly the R325 protein variant. The polymorphism is common in individuals of European and East Asian descents and associated with a $14 \%$ increase in diabetes incidence per risk allele (Cauchi et al., 2010). The first functional studies demonstrated that the R325 risk variant is less active than the low-risk W325 variant (Nicolson et al., 2009; Kim et al., 2011) and provided evidence that ZnT8 activation could minimize diabetes risk. In fact, SLC30A8 overexpression in insulinoma cells enhanced insulin secretion during hyperglycemia. Thus, ZnT8 may promote zinc storage in insulin granules and thereby the insulin secretory pathway (Chimienti et al., 2006; Sladek et al., 2007). The ability to transport and store zinc rather than the extracellular zinc status is important for zinc accumulation in $\beta$-cells. Therefore, SLC30A8 overexpression could counteract zinc depletion in diabetes, which likely participates in $\beta$-cell mass loss (Chimienti et al., 2006). According to the effect of $S L C 30 A 8$ overexpression on insulin secretion, subjects homozygous for the C-risk allele exhibit a decrease in first-phase insulin release after intravenous glucose administration (Boesgaard et al., 2008).

Influences of endogenous plasma zinc and dietary zinc supplementation on insulin secretion or T2D risk have been related to $S L C 30 A 8$ sequence variants. Glucose-stimulated insulin secretion in healthy nondiabetic Amish individuals with and without the rs13266634 polymorphism was tested before and after zinc supplementation. Contrary to the previous hypothesis, individuals with the lower-risk CT/TT genotypes showed attenuated insulin responses to glucose stimulation compared with homozygous C-risk allele carriers without zinc supplementation (Maruthur et al., 2015). However, after 14 days of zinc supplementation, the increase in insulin response to glucose was not significantly changed in the homozygous risk allele carriers compared with baseline and was increased further in the nonrisk CT/TT-allele carriers (Maruthur et al., 2015). A prospective study of a Swedish cohort presents zinc supplementation as lowering the risk of T2D, but does not find significant modification of the zinc-T2D associations by the SLC30A8 rs13266634 polymorphism (Drake et al., 2017). However, T2D risk was lowest among nonrisk allele carriers on zinc supplements. A Chinese population presented the inverse association between plasma zinc levels and diabetes. The association was more pronounced among individuals carrying the TT genotype of SLC30A8 rs13266634 polymorphism than those carrying CT or CC genotypes (Shan et al., 2014). The attenuated inverse association of zinc level and T2D risk in the subjects carrying the C-risk allele supports the data sets on zinc supplementation (Maruthur et al., 2015; Drake et al., 2017). Apparently, beneficial zinc effects on metabolism are attenuated in risk allele carriers.
Although the first functional studies of the SLC30A8 risk allele discovered in 2007 predicted reduced transporter activity, SLC30A8 sequence variants lowering diabetes risk were described in 2014 (Flannick et al., 2014). Twelve novel SLC30A8 sequence variants, resulting in a truncated protein and loss of transporter activity, predicted reduced T2D risk. This publication was groundbreaking because previous functional studies of ZnT8 suggested that reduced zinc transport increases T2D risk, whereas SLC30A8 loss-of-function mutations provide strong evidence that haploinsufficiency protects against $\mathrm{T} 2 \mathrm{D}$, and $\mathrm{ZnT} 8$ inhibition may be suitable to treat T2D. However, the novel identified variants are rare.

c. Metabolic phenotype of animal models. The potential causal role of ZnT8 in T2D has been extensively investigated in mouse models (Table 1). HFD-fed Slc30a8 knockout mice are glucose intolerant and diabetic with less responsive $\beta$-cells (Lemaire et al., 2009). Similarly, chow-fed Slc30a8 knockout mice showed reduced glucose-stimulated insulin secretion and lower zinc content of secretory granules, but an overall mild metabolic phenotype (Nicolson et al., 2009; Pound et al., 2009). Because ZnT8 is not only expressed in $\beta$-cells, but also in pancreatic $\alpha$-cells (Nicolson et al., 2009), the role of $\mathrm{ZnT} 8$ in the two different islet cell populations was further addressed using a $\beta$ cell-specific Slc30a8 knockout mouse. HFD-fed wholebody Slc30a 8 knockout mice became remarkably obese, hyperglycemic, hyperinsulinemic, insulin resistant, and glucose intolerant, whereas mice with specific Slc30a8 deletion in $\beta$-cells showed an attenuated metabolic phenotype with a similar body weight to control mice (Hardy et al., 2012). These data suggest that ZnT8 contributes to the risk of developing T2D through $\beta$-cell- and non- $\beta$-cell-specific effects (Hardy et al., 2012). Indeed, the metabolic function of $\mathrm{ZnT} 8$ and interacting environmental factors, genetic background, gender, and age ultimately determine the variable metabolic phenotype of whole-body Slc30a8 knockout (Lemaire et al., 2009; Nicolson et al., 2009; Pound et al., 2009,2012 ) and $\beta$-cell-specific Slc30a 8 knockout models (Wijesekara et al., 2010; Hardy et al., 2012; Tamaki et al., 2013; Mitchell et al., 2016). However, most of the studies display abnormalities in insulin crystallization and storage, impaired glucose tolerance, and lower circulating insulin levels. Thus, reduced ZnT8 activity alters glucose homeostasis, thereby increasing T2D risk (da Silva Xavier et al., 2013). Vice versa, Slc30a8 transgenic mice overexpressing the transporter in $\beta$-cells show markedly improved glucose tolerance (Mitchell et al., 2016).

Unexpectedly, some studies showed unaltered or improved insulin release after glucose stimulation of isolated Slc30a8 knockout $\beta$-cells (Lemaire et al., 2009; Nicolson et al., 2009; Tamaki et al., 2013; Mitchell et al., 2016). The contradictory observation of lower plasma 
insulin levels in vivo, but improved insulin secretion in vitro, could be explained by a functional link between Slc30a 8 deletion in $\beta$-cells and hepatic insulin clearance (Tamaki et al., 2013). Zinc secreted along with insulin from pancreatic secretory granules could conceivably affect neighboring endocrine cells and the liver. By inhibiting clathrin-dependent insulin endocytosis, zinc suppresses hepatic insulin clearance. Insulin uptake in hepatocytes increases as a result of the lower pancreatic zinc release in Slc30a8 knockout mice. Indeed, the study also showed low circulating insulin levels in the face of insulin hypersecretion from pancreatic $\beta$-cells. Because ZnT8 affects hepatic insulin clearance, genetic dysregulation of this could contribute to T2D.

HFD-fed transgenic mice overexpressing the human SLC30A8 high-risk R325 variant exhibit increased pancreatic zinc and proinsulin levels and decreased insulin and glucose tolerance, confirming the detrimental effect of the human risk allele (Li et al., 2017a). Conversely, overexpression of the low-risk W325 variant decreases pancreatic zinc and proinsulin levels together with increases in insulin and glucose tolerance. The beneficial metabolic response to SLC30A8 overexpression strongly suggests that $\mathrm{ZnT} 8$ activation could ameliorate metabolic disease. This idea is further supported by the fact that pancreatic $\mathrm{ZnT}$ \% protein levels are downregulated in diabetic disease models such as $d b / d b$ and Akita mice in the early stages of T2D (Tamaki et al., 2009).

In contrast to previously described mouse phenotypes and gene expression data stressing potential benefits of $\mathrm{ZnT}$ 8 activation, a recent study of knockin mice carrying a human SLC30A8 loss-of-function variant yielded unexpected findings. The model featured increased glucose-induced insulin secretion, likely reflecting increased capacity of $\beta$-cells to secrete insulin. The study suggested for the first time that ZnT8 inhibition could elicit beneficial metabolic responses (Kleiner et al., 2018), supporting the finding of SLC30A8 loss-offunction variants that reduced T2D risk (Flannick et al., 2014). Although loss of function seems to be beneficial in this mouse line, the variability of mouse phenotypes makes it difficult to judge how ZnT8 must be modulated to achieve metabolic protection.

d. Pharmacological activation or inhibition of zinc transporter 8 . The diabetic phenotypes of the knockout mice and human data on SLC $30 A 8$ genetics and zinc supplementation support the idea that ZnT8 augmentation could improve T2D. However, discrepant data concerning the metabolic effect of the risk allele raise the question of whether the $S L C 30 A 8$ risk variant causes an increase or a decrease in zinc transport activity. Accordingly, whether ZnT8 activation or inhibition should be pursued as T2D treatment remains unclear. Whereas in mice loss of ZnT8 function results in impaired glucose tolerance, human data are contradictory, with studies describing high-risk and low-risk variants with loss of function. The SLC30A8 R325 risk variant displayed lower zinc transport (Nicolson et al., 2009), but was proposed to be hyperactive in another study (Merriman et al., 2016) that is supported by the finding that the diabetes risk genotype $\mathrm{CC}$ at rs13266634 is associated with higher islet zinc concentrations (Wong et al., 2017), along with the identification of rare T2D-risk-reducing SLC30A8 loss-of-function mutations (Flannick et al., 2014). The following concept may explain the paradoxical finding that high-risk and low-risk variants both present lower protein activity (Rutter and Chimienti, 2015): assuming the activity of the risk variant is $30 \%$ lower compared with the nonrisk variant, zinc transport into granules would be reduced by $15 \%$ and $30 \%$ in heterozygous and homozygous C-allele carriers. In contrast, heterozygous loss-of-function variants result in 50\% lower activity. Consequently, the rare loss-of-function mutations result in a lower transport activity compared with the $r s 13266634$ risk variant. The risk variant R325 results in moderate reduction of $\mathrm{ZnT} 8$ activity, lowering $\beta$-cell zinc secretion and enhancing hepatic insulin clearance. In contrast, more severe reduction in ZnT8 function increases insulin secretion from $\beta$-cells, compensating the impaired zinc release and insulin clearance. This model argues for a complex doseresponse relationship between $\mathrm{ZnT} 8$ activity and diabetes risk (Rutter and Chimienti, 2015). Speciesspecific differences may explain the increased risk of hyperglycemia in Slc30a8 knockout mice with the simpler model of progressively impaired insulin action due to increased hepatic clearance. Moreover, the differential role of ZnT8 in insulin secretion and glucose homeostasis could be explained in part by an age-dependent effect (Rutter and Chimienti, 2015). This idea originated from the observation of impaired glucose tolerance and abnormal insulin granules in younger (6-8 weeks old), but not in older Slc30a8 knockout mice (Nicolson et al., 2009). Furthermore, pancreatic islet cell survival in the face of hypoxia was enhanced in older ( $>12$ weeks) Slc30a 8 knockout mice, but not younger animals (Gerber et al., 2014). However, whether findings in rodents can be translated to human disease remains unclear, and particularly the interaction with age, gender, drugs, or physical activity may influence ZnT8 function.

Taken together, both rodent and human data highlight the significance of ZnT8 function in glucose homeostasis and the pathological consequences of $\mathrm{ZnT} 8$ malfunction. However, drug interventions targeting $\mathrm{ZnT}$ \% are likely complex given the proposed bell-shaped model of the of ZnT8 function in the development of T2D. Clearly, effects of both activating and inhibiting drugs will need to be investigated in suitable models prior to clinical trials in human beings (Rutter and Chimienti, 2015).

2. Therapeutic Potential of Solute Carrier 30 Transporters. Because zinc transporters are crucial 
for zinc homeostasis, any dysregulation could promote development of metabolic disease, particularly diabetes mellitus. Regarding existing data on the SLC30 family of zinc transporters, SLC30A3/ZnT3 is linked to insulin secretion and SLC30A7/ZnT7 shows an association with diet-induced obesity and insulin resistance in mice (Table 2). Both SLC30 members will be discussed in Emerging Potential of Other Solute Carrier Target Structures. Finally, SLC30A8 is the only candidate gene that has been linked to human T2D in genetic studies and is therefore the most promising target for a therapeutic intervention (Table 1). Research on ZnT8 is ongoing; since 2018, multiple studies regarding T2D susceptibility in different populations have been published, highlighting the critical role of this zinc transporter in the onset of human diseases (Ding et al., 2018; Dong et al., 2018; Li et al., 2018; Lin et al., 2018; Liu et al., 2018; Plengvidhya et al., 2018). Further studies are needed to finally verify whether increased or decreased transporter activity is linked to human T2D, and therefore whether inhibition or rather activation of the transporter is required to counteract disease development. However, ZnT8 seems to be a promising therapeutic target for the treatment of T2D and potentially also related metabolic diseases, and should therefore be investigated further.

\section{Proposed Requirements for Metabolic Target Structures}

In this review, we evaluate metabolic target structures by the available data supporting their role in metabolic regulation and exploitability in human metabolic disease (Tables 1 and 2). A protein may be regarded as a potential target if its genetic locus and protein function in the setting of metabolic disease are established in cellular systems, animal models, and human beings. Cell-based studies can point to effects of specific targets on insulin secretion [e.g., ZnT3 (Smidt et al., 2009)], glucose uptake [e.g., SGLT1 and SGLT2 (Ghezzi et al., 2018)], or oxygen consumption [UCP1 (Ost et al., 2017)]. Ideally, an animal model then substantiates the effect by showing relevance in a disease model of genetic and diet-induced obesity, insulin resistance, NAFLD, and $\beta$-cell loss. Metabolic effects of transporter modulation should be investigated in animal models such as the obese insulin-resistant $o b / o b$ or diabetic $d b / d b$ mice, or knockout mice. However, the classification of a metabolic target also requires human validation because it has become clear that a large part of the available animal data does not translate into the human setting (Perel et al., 2007; Mak et al., 2014). Ideally, either GWA or MR studies should validate and substantiate experimental data from cells and animals. However, functionally relevant polymorphisms may be too rare, and the number of patients needed to show conclusive, statistically significant results in GWAS or MR, after adjusting for multiple testing, may exceed the size of existing cohorts. Therefore, studies using human specimens, disease-related regulation of gene expression, or functional changes in the setting of metabolic disease may complement the picture consisting of cellular, systemic animal and human data.

A prime example for our proposed requirements is the SLC5 family member SGLT2, which fulfilled all defined criteria and is to date one of the preferred targets with which to treat T2D: 1) SGLT2 has been shown to mediate glucose uptake in cellular systems (Ghezzi and Wright, 2012); 2) animal models lacking SGLT2 were protected from diet-induced and genetic obesity, T2D, and $\beta$-cell loss (Jurczak et al., 2011, 2018); and 3) mutations in humans provided evidence for a clear role of SGLT2 in renal glucose handling (Santer et al., 2000, 2003; van den Heuvel et al., 2002). Finally, the SGLT2 inhibitor phlorizin was available and was proven beneficial in the setting of diabetes in animals (Ehrenkranz et al., 2005). Together, these data resulted in the development of clinically available SGLT2 inhibitors for the treatment of T2D, which have not only shown their potency and efficacy in reducing glycemia, but also in mediating cardiovascular benefit such as reduction in heart failure hospitalization and progression of kidney disease in patients with and without pre-established cardiovascular disease (Hanefeld et al., 2016; Birkenfeld et al., 2019; Zelniker et al., 2019).

\section{Emerging Potential of Other Solute Carrier Target Structures}

This review aims to elucidate members of the SLC superfamily that are linked to human metabolic disease and could serve as treatment targets. The potential and relevance of the transporters in metabolic function and dysfunction were gauged based on existing human and mouse data. Some associations of transporterencoding SLC gene variants are very strong and are underpinned by GWAS or other genome analyses, as well as by phenotype-related mouse models. Transporters that show a clear link in human metabolic disease or at least some link combined with strong supporting mouse data were presented in Links to Human Metabolic Disease and Potential Targets and Table 1. Other SLC genes of the selected families are linked to metabolic disorders only in rodent models or are only marginally linked to metabolic disease in humans. The SLC members presented in this section include those with the potential for future research; however, existing data are not to date sufficiently conclusive to propose the transporter as a target to treat metabolic disease. An overview of these SLC members is presented in Table 2.

\section{A. Solute Carrier 5 Family}

SLC5A1/SGLT1 and SLC5A2/SGLT2 are the most prominent members of the SLC5 family given their 
close association with metabolic disease (Table 1) and drugs targeting the transporters showing benefits in multiple clinical trials. However, other SLC5 family members may have potential in the management of metabolic disease. These candidates, such as SLC5A9/ SGLT4 and SLC5A10/SGLT5, are linked to diabetic retinopathy and hepatic steatosis (Table 2).

1. Solute Carrier 5A9/Sodium-Glucose Cotransporter 4. SGLT4, encoded by the SLCA9 gene, is a transporter for mannose, glucose, and fructose primarily expressed in the intestine, but also in the kidney and liver (Tazawa et al., 2005). SGLT4 likely contributes to intestinal absorption and renal reabsorption of sugar analogs, including mannose, but also 1,5anhydro-D-glucitol. Elevated mannose serum concentrations are associated with diabetes mellitus and the metabolic syndrome (Pitkänen, 1996; Pitkänen et al., 1999). Conversely, 1,5-anhydro-D-glucitol serum concentrations are reduced in diabetic patients (Servo and Pitkänen, 1975; Akanuma et al., 1981; Yamanouchi et al., 1991; Pitkänen, 1996). The SLC5A9 gene mutations may contribute to changes in serum metabolites seen in diabetic patients (Tazawa et al., 2005). A recent study suggested an association between three rare SLC5A9 variants-rs149485404, rs775853981, and rs61997217-and proliferative diabetic retinopathy (Ung et al., 2017), which is a common complication of diabetes and a leading cause of blindness worldwide. However, the study cohort only comprised a limited number of patients. Taken together, human data are scarce, and, until now, SLC5A9 variants or mutations have not directly been linked to diabetes and metabolic syndrome. A Slc5a9-deficient mouse model could prove the hypothesis of physiological implications in T2D and related clinical complications.

2. Solute Carrier 5A10/Sodium-Glucose Cotransporter 5. SGLT5, product of the SLC5A10 gene, is exclusively expressed in the kidney and transports mannose and fructose with high capacity and to a lesser extent glucose and galactose (Grempler et al., 2012). Physiologically, SGLT5 mediates the reabsorption of its substrates from urine into renal proximal tubule cells. The SLC5A10 variant rs117355297 and two rare lossof-function mutations are significantly associated with circulating 1,5-anhydro-D-glucitol ( $\mathrm{Li}$ et al., 2017b; Long et al., 2017), an accepted biomarker heralding diabetic complications (Parrinello and Selvin, 2014).

Slc5a10 knockout mice showed increased urinary fructose, indicating that SGLT5 is important for renal fructose reabsorption (Fukuzawa et al., 2013). Because high fructose intake is epidemiologically associated with dyslipidemia, obesity, NAFLD, diabetes, and cardiovascular disease (Johnson et al., 2007; Stanhope and Havel, 2010), the authors hypothesized that SGLT5 deficiency could protect from fructose-induced hepatic steatosis. Surprisingly, SGLT5 deficiency worsened fructoseinduced hepatic steatosis, plasma transaminases, and fasting hyperinsulinemia in another study (Fukuzawa et al., 2013). To date, the transporter has not been related to human disease, but mouse data, although conflictive, suggest that the contribution of SGLT5 in fatty liver disease deserves to be studied in more detail.

\section{B. Solute Carrier 13 Family}

Three additional SLC13 transporters might have potential besides the promising candidate SLC13A5/ NaCT (Tables 1 and 2). SLC13A2/NaDC1 and SLC13A3/NaDC3 are associated with renal function and disease in GWAS and could be further investigated as potential targets to treat diabetic nephropathy or other kidney diseases. Moreover, SLC13A1/ NaS1 has been linked to fatty liver disease in a welldescribed knockout mouse model. However, their potential in the context of obesity or T2D has not been sufficiently examined.

1. Solute Carrier 13A1/Sodium-Coupled Sulfate Cotransporter 1. NaS1, product of the SLC13A1 gene, mediates the absorption of sulfate, an essential micronutrient required for various physiological processes, in the small intestine and its reabsorption in the kidney, thereby regulating sulfate plasma level and sulfate homeostasis (Markovich, 2001; Markovich and Murer, 2004). The two single-nucleotide variants, rs 28364172 and $r s 138275989$, were shown to be strongly associated with low serum sulfate level, a so-called hyposulfatemia, as well as the high serum level of the liver transaminases alanine aminotransferase and aspartate aminotransferase (Tise et al., 2016). A potentially detrimental effect of disturbed sulfate homeostasis on liver metabolism is supported by the hyposulfatemic Slc13a1 knockout mouse model showing altered hepatic gene expression associated with increased hepatic lipid, serum cholesterol, and low-density lipoprotein concentration, as well as reduced hepatic glycogen content (Dawson et al., 2006). Although human NaS1 is not convincingly related to metabolic disease patterns, rodent data support the hypothesis that NaS1 activation might be metabolically beneficial. However, because NaS1 interferes with multiple physiological processes, as evidenced by neurological and behavioral abnormalities, enhanced tumor growth, and reduced fertility in Slc13a1 knockout mice (Dawson et al., 2003, $2004,2005,2010,2011$ ), consequences of NaS1 modulation seem complex, and the idea and consequences of transporter modulation need further investigation.

2. Solute Carrier 13A2/Sodium-Coupled Di- and Tricarboxylate Transporter 1. NaDC1, encoded by the $S L C 13 A 2$ gene, is mainly expressed in the apical membrane of renal proximal tubular and small intestine cells, where it reabsorbs TCA cycle intermediates such as succinate, $\alpha$-ketoglutarate, and citrate, thereby providing substrates for cells with high metabolic needs (Chen et al., 1998). The key physiological role of the NaDC1 transporter is the regulation of 
urinary citrate excretion. An important function of citrate in the urine is complexing calcium and thereby preventing calcium crystallization and precipitation (Bek-Jensen et al., 1996). Low urinary citrate excretion, a so-called hypocitraturia, increases the risk of nephrolithiasis, the formation of calcium-containing renal stones (Kleeman et al., 1980; Rudman et al., 1982; Nicar et al., 1983; Hamm, 1990). Findings in human beings and rodents relate NaDC1 to nephrolithiasis (Table 2). Notably, links between nephrolithiasis and metabolic disease have been extensively discussed. For example, the hazard ratio for new-onset T2D increased in patients with renal stones (Chung et al., 2011). Additional studies highlighting the link between nephrolithiasis and the metabolic syndrome, cardiovascular disease, hypertension, and diabetes have been recently reviewed (Ramaswamy and Shah, 2014).

The A-allele of the SLC13A2 polymorphism rs11567842 (c.1648A $>$ G; p.I550V) is associated with reduced urinary citrate concentrations (Okamoto et al., 2007), whereas the G-allele variant is significantly associated with increased urinary citrate excretion, suggesting that AA-allele carriers are more susceptible to hypocitraturia, which increases their risk for renal stone recurrence (Udomsilp et al., 2018). No significant association of the polymorphism with presence of renal stone formation was observed, indicating that the SNP itself may not be sufficient to affect formation of kidney stones. In the presence of additional metabolic risks, however, nephrolithiasis may occur earlier in A-allele-carrying subjects (Okamoto et al., 2007). The authors suggest that patients with recurrent kidney stones carrying the A-allele of the rs11567842 polymorphism might benefit in terms of nephrolithiasis risk from citrate-rich diets to increase urinary citrate excretion.

Slc13a2 knockout mice excreted significantly larger amounts of TCA cycle intermediates in urine (Ho et al., 2007), confirming the human data (Okamoto et al., 2007; Pajor and Sun, 2010; Udomsilp et al., 2018). However, in this model, renal function was not affected under normal or ischemic conditions and no additional metabolic changes were observed. In rats, Slc13a2 expression was increased on renal proximal tubule epithelial cells in a model of chemically-induced nephrolithiasis (He et al., 2004b). Nevertheless, renal NaDC1 expression was reduced in a rat model of obstructive nephropathy, which can be caused by nephrolithiasis (Campagno et al., 2018). Finally, the opposing regulation of Slc13a2 in different animal models needs further evaluation.

NaDC1 has also been hypothetically implicated in the pathogenesis of arterial hypertension (Bergeron et al., 2013). The idea is based on the knowledge that the renal function and blood pressure regulating serum and glucocorticoid-regulated kinase 1 signaling pathway stimulates $\mathrm{NaDC} 1$, and succinate, also a $\mathrm{NaDC} 1$ substrate, increases blood pressure in animals. This effect is absent in G protein-coupled receptor 91 (succinate receptor)-deficient mice (Boehmer et al., 2004; He et al., 2004a; Bergeron et al., 2013). However, a direct link between $\mathrm{NaDC} 1$ function and arterial hypertension has not yet been proven.

Taken together, NaDC1 appears to affect susceptibility to develop nephrolithiasis. Mechanistically, SLC13A2 polymorphisms in humans with decreased transporter function and genetic Slc13a2 deletion in mice both raise urinary citrate levels. Because citrate attenuates renal stone formation, NaDC1 inhibition could be therapeutically useful against nephrolithiasis. Although nephrolithiasis has been linked to diabetes (Chung et al., 2011; Ramaswamy and Shah, 2014), it is questionable whether NaDC1 targeting has the potential to treat metabolic disease. In fact, SLC13A2 polymorphisms have not been directly linked to obesity, diabetes, insulin resistance, or fatty liver in human beings. Further investigations, especially in mouse models of diet-induced obesity, are needed to verify the potential of $\mathrm{NaDC} 1$.

3. Solute Carrier 13A3/Sodium-Coupled Di- and Tricarboxylate Transporter 3 . NaDC3, encoded by the SLC13A3 gene, is primarily expressed in the kidney, liver, and brain (Fig. 2) and exhibits substrate specificities similar to those of NaDC1 (Fig. 1) (Chen et al., 1999). The most physiologically important NaDC3 substrate is $\alpha$-ketoglutarate. In the kidney and liver, NaDC3 is expressed on the basolateral membrane supplying TCA cycle intermediates for mitochondrial energy metabolism. The substrates may also be used for gluconeogenesis (Chen et al., 1999). Furthermore, in the liver, NaDC3 likely plays a role in glutamine and ammonia metabolism. In the kidney, NaDC3 is involved in organic anion secretion by taking up $\alpha$-ketoglutarate from blood (Bergeron et al., 2013). Importantly, NaDC3 acts in concert with the organic anion transporter OAT2 (SLC22A7), which facilitates the import of organic anions in exchange for dicarboxylates (Bergeron et al., 2013). Through this interaction, both transporters regulate the secretion of organic anions. In the brain, the transporter is mainly expressed in astrocytes, but not in neurons. It is supposed that supply with dicarboxylates is mediated by NaDC3 in astrocytes and $\mathrm{NaCT}$ in neurons (Bergeron et al., 2013).

The two SLC13A3 SNPs, rs126917 and rs2425885, have been linked to T2D and end-stage renal disease (Bento et al., 2008). However, the identified polymorphisms are possibly associated with diabetic nephropathy rather than T2D, and therefore, similar to NaDC1, further studies of patients with diabetes, but without nephropathy, are needed to clarify disease specificity. Another GWAS yielded a significant association for the SLC13A3 polymorphism rs6066043 and chronic kidney disease of unknown etiology (Nanayakkara et al., 2014). Indeed, renal Slc13a3 expression was positively 
correlated with progressive renal fibrosis in mice as well as in kidney biopsies obtained from patients with stage III/IV chronic kidney disease, suggesting SLC13A3 gene and protein expression as a potential molecular biomarker for disease progression (Ju et al., 2009).

Besides the presented renal disease patterns, the SLC13A3 polymorphism rs6066029 was linked to arterial hypertension (Simino et al., 2011). The authors proposed "suggestive" associations between blood pressure and the rs6066029 variant, defined by a less stringent genome-wide significance threshold compared with the conventional statistical significance threshold that is conservative for limited sample size or rare variants (Simino et al., 2011). Additionally, NaDC3 may also be involved in the pathogenesis of inborn metabolic diseases, including glutaric aciduria type 1, Canavan disease, and Axenfeld-Rieger syndrome type 1 (Bergeron et al., 2013).

Taken together, existing human genetic association data are scarce and inconclusive; strong genotype phenotype correlation is not shown in larger cohorts. Nevertheless, several studies have linked NaDC3 to diabetes, hypertension, and, most importantly, renal disease (Table 2). Because direct links between NaDC3 and diabetes have not been established, further GWAS and valid animal models are required to validate a causative link. It remains to be seen whether NaDC3 could serve as pharmacological target for the treatment of metabolic disease.

\section{Solute Carrier 16 Family}

Besides the extensively discussed potential targets SLC16A1/MCT1, SLC16A11/MCT11, and SLC16A13/ MCT13 (Table 1), additional SLC16 transporter might be interesting in the context of metabolic disease. SLC16A3/MCT4 has been linked to diabetic nephropathy, and SLC16A6/MCT7, SLC16A9/MCT9, and SLC16A10/MCT10 are marginally linked to metabolic phenotypes in single animal or human studies (Table 2).

1. Solute Carrier 16A3 / Monocarboxylate Transporter 4. MCT4, encoded by the SLC16A3 gene highly expressed in the skeletal muscle, brain, and intestine, is a well-characterized proton-coupled monocarboxylate transporter whose pathophysiology, similarly to MCT1, has mainly been studied in cancer research (Jones and Morris, 2016; Fisel et al., 2018). Interestingly, SLC16A3 was linked to diabetic nephropathy, a common complication of diabetes, in a gene expression study in ethnic Malays with T2D. SLC16A3 overexpression was described in patients with diabetes and renal impairment, suggesting that SLC16A3 may confer susceptibility for diabetic nephropathy (Lokman et al., 2011). SLC16A3 gene expression was also studied in patients with obesity; SLC16A3 tended to be overexpressed in patients with obesity before weight loss and slightly decreased after weight loss (Metz et al., 2008). However, for both human studies, the number of patients was limited, and large-scale studies need to confirm the associations to diabetic nephropathy and obesity. Further investigations, including the characterization of a Slc16a3-deficient mouse line, are needed to evaluate the real potential of this transporter.

2. Other Solute Carrier 16 Transporters. Other SLC16 family members are less characterized and only marginally described in the context of metabolic disease. MCT7, encoded by the SLC16A6 gene, transports ketone bodies and is broadly expressed. A Slc16a6 mutation was identified in zebrafish mutants with hepatic steatosis. The phenotype could be rescued by transgenic expression of the human ortholog SLC16A6 (Hugo et al., 2012). However, rodent and human data linking SLC16A6 expression and fatty liver are lacking. SLC16A9, coding for the carnitine transporter MCT9, is mainly expressed in the kidney and adrenal glands. The SLC16A9 SNP rs12356193 is associated with serum uric acid levels (Kolz et al., 2009; van der Harst et al., 2010; Köttgen et al., 2013). Elevated serum uric acid levels are risk factors for gout and associated with cardiovascular disease and diabetes. Another sequence variant of the gene, rs2242206, was associated with renal overload gout, but not with overall gout susceptibility (Nakayama et al., 2013). MCT10, encoded by SLC16A10, transports aromatic amino acids and is highly expressed in skeletal muscle and in the heart. One genome study addressing branched-chain amino acid metabolism in nonalcoholic fatty liver disease linked MCT10 to NAFLD with reduction in SLC16A10 gene expression in human disease development (Lake et al., 2015). However, additional genetic studies in larger populations and detailed knowledge on cellular mechanisms are missing. Therefore, the potential of MCT7, MCT9, and MCT10 in clinical therapies to treat gout, fatty liver, and associated metabolic diseases needs to be further investigated. Knockout mouse strains would also be of great interest for these genes.

\section{Solute Carrier 25 Family}

Mitochondrial carriers are diverse in their substrate specificity, and many are linked to human diseases (Table 1). In addition to the discussed potential targets, including the prominent uncoupling protein SLC25A7/ UCP1, SLC25A25/APC3 and SLC25A40/MCFP are associated with diet-induced obesity in mice and hypertriglyceridemia in humans, respectively (Table 2).

1. Solute Carrier 25A25 / ATP-Mg / Phosphate Carrier Protein 3. SLC25A25 encodes the mitochondrial APC3. It is ubiquitously expressed and, like APC1, member of the subfamily of mitochondrial ATP-Mg/Pi carrier proteins. The transporter has been characterized in terms of metabolic function in Slc25a25 knockout mice. The model exhibits resistance to diet-induced obesity as well as reduced physical endurance (Anunciado-Koza et al., 2011). Strikingly, the authors also found Slc25a25 to be upregulated in skeletal muscle and inguinal fat in 
cold-adapted Slc25a7-deficient mice. Accordingly, Slc25a25 overexpression and deletion both result in reduced metabolic efficiency. APC3 is proposed to maintain ATP homeostasis by functioning as a $\mathrm{Ca}^{2+}$-regulated shuttle of ATP- $\mathrm{Mg}^{2+}$ and phosphate, potentially resulting in impaired capacity for ATP synthesis necessary to support $\mathrm{Ca}^{2+}$ cycling in skeletal muscle. Loss of APC3 function may result in less efficient muscular activity, shown by reduced exercise endurance on a treadmill, and therefore, Slc25a25 knockout mice require more caloric energy, which reduces fat stores and body weight (Anunciado-Koza et al., 2011). Although human genetic association data or mechanistic explanations linking APC3 to metabolic disease are missing, the transporter appears to be a promising candidate for further rodent and human studies exploring its contribution to energy metabolism and potential therapeutic applications.

2. Solute Carrier 25A40/Mitochondrial Carrier Family Protein. SLC25A40 is ubiquitously expressed at low levels and encodes the MCFP as one of the orphan transporters of the SLC25 family with unknown substrate specificity. The SLC25A40 single-nucleotide variant rs762174003, probably resulting in reduced protein function, is significantly associated with high circulating triglyceride levels, a common risk factor for T2D and cardiovascular diseases (Rosenthal et al., 2013). Another recent study addressed mechanisms of mitochondrial dysfunction after cardiopulmonary bypass in patients with diabetes. SLC25A40, as well as PGC1 $\alpha$, was significantly downregulated after cardiopulmonary bypass in patients with diabetes, together with lower levels of antioxidant marker and angiogenic and antiapoptotic factors (Mahmood et al., 2019). In this context, MCFP may contribute to mitochondrial function. However, MCFP has not been functionally characterized or investigated using mouse models; as such, its metabolic role and links to hypertriglyceridemia and mitochondrial function in diabetes remain unclear.

\section{E. Solute Carrier 30 Family}

Within the SLC30 family, two members additional to SLC30A8/ZnT8 (Table 1) are linked to metabolic abnormalities in rodents: SLC30A3/ZnT3 and SLC30A7/ ZnT7 (Table 2). However, their therapeutic potential is unassigned because genome-wide association in humans is missing. Other SLC30 genes are to date only reported to be differentially expressed in obese subjects.

1. Solute Carrier 30A3/Zinc Transporter 3. $\mathrm{ZnT}$, encoded by the SLC30A3 gene, is almost exclusively localized in the brain and essential for zinc transport into synaptic vesicles (Palmiter et al., 1996). Slc30a3 knockdown in insulinoma cells lowered insulin secretion, and knockout in a streptozotocin-induced diabetes mouse model reduced glucose tolerance (Smidt et al., 2009). In rats, altered Slc30a3 expression was associated with fat pad weight (Keele et al., 2018). SLC30A3 polymorphisms have not been linked to human metabolic disease to date, rather to schizophrenia and Alzheimer's disease (Rovelet-Lecrux et al., 2012; Perez-Becerril et al., 2014). However, one human study linked lower SLC30A3 expression in adipocytes to obesity (Smidt et al., 2007). Regarding existing cellbased mouse and human data, although scarce, $\mathrm{ZnT3}$ function is worth studying in more detail in the context of metabolic disease.

\section{Solute Carrier 30A7/Zinc Transporter 7.} $\mathrm{ZnT7}$, encoded by the SLC30A7 gene, is mainly expressed in the small intestine and mediates zinc transport from the cytoplasm into the Golgi apparatus (Kirschke and Huang, 2003). Slc30a7 knockout mice showed a reduction in food intake, growth, and body fat, and slightly elevated glucose levels after oral glucose administration (Huang et al., 2007). Interestingly, $S l c 30 a 7$ is also expressed in the mouse pancreas, and its overexpression in insulin-secreting $\beta$-cells elevates insulin synthesis and secretion (Huang et al., 2010). Diet-induced obese Slc30a 7 knockout mice are prone to metabolic changes, including glucose intolerance and insulin resistance. The finding provides in vivo evidence for a direct role of $\mathrm{ZnT7}$ in the regulation of glucose homeostasis (Huang et al., 2012). Insulin resistance in this model was primarily associated with increased intracellular fatty acid levels in skeletal muscle, promoting intracellular lipid accumulation and insulin resistance (Huang et al., 2018). In diabetic rats, hyperglycemia-induced alteration of $\mathrm{ZnT7}$ activity causes zinc release from the sarco(endo)plasmic reticulum and mediates endoplasmic reticulum stress in the heart, which might contribute to cardiac dysfunction in diabetes (Tuncay et al., 2017, 2019). No functional characterization of $\mathrm{ZnT} 7$ and link to diabetes in humans has yet been described, but mouse data are promising.

3. Other Solute Carrier 30 Transporters. Less evidence for an association with metabolic disease is present for other transporters of the family. Differential gene expression in human visceral and subcutaneous adipocytes between lean and obese subjects was shown for SLC30A2 and SLC30A6 with a lower expression in obese subjects, suggesting that obesity can influence the adipocyte zinc-transporting system or vice versa (Smidt et al., 2007). Another study, also addressing the mechanisms controlling zinc metabolism in obesity, analyzed expression of the SLC30 genes in leukocytes and presented significantly lower SLC30A4, SLC30A5, and SLC30A9 levels in leukocytes of obese women and the inversely correlated expression of the genes with BMI and body fat (Noh et al., 2014). However, no rodent and human genetic data indicate the link to obesity-related metabolic disorders, and the available human expression sets are poor, but there are existing hints that these transporters might be deregulated in metabolic disease and considered as therapeutic target. Within the SLC30 family and based on mouse data, SLC30A3 and SLC30A7 
seem to be the most interesting candidates besides SLC30A8.

In conclusion, additional members of the SLC5, SLC13, SLC16, SLC25, and SLC30 families were identified to be associated with disease patterns in humans and/or mice. Whether modulation of these transporters could have any therapeutic potential remains elusive. However, although links to human metabolic disease are weak for these candidates, raising the prevalence of diabetes and related metabolic diseases requires novel medical approaches, and future research on these SLC members shows promise.

\section{Conclusion and Outlook}

In this review, we presented transporters of the SLC superfamily that are linked to human metabolic disease and may therefore be of interest for therapeutic intervention. Remarkably, SGLT2 fulfills a special role because it is, within the SLCs, the only already validated drug target to treat metabolic disease. Currently, four SGLT2 inhibitors are FDA-approved: canagliflozin, dapagliflozin, empagliflozin, and ertugliflozin. Importantly, SGLT2 inhibition was shown to lower glucose levels, body weight, blood pressure, and liver fat in clinical trials.

Two other SLC transporters have been linked to metabolic disease in humans and/or mice and are simultaneously targeted by drugs in clinical trials. However, the ongoing clinical trials do not aim to treat metabolic disease and/or target the SLC specifically. The SLC16A1/MCT1 inhibitor AZD3965 is in clinical testing as an anticancer agent. MCT1 is linked to exercise-induced hyperinsulinemia in humans and obesity, insulin resistance, and hepatic steatosis in mice, and therefore reflects a candidate with high potential. Furthermore, linifanib (ABT-869), an inhibitor of receptor tyrosine kinases, which is also in clinical testing to treat different types of tumors, was most recently identified as a potent inducer of SLC25A7/UCP1. The essential role of UCP1 in body weight regulation and diabetes is presented in various mouse and human studies, and mitochondrial uncoupling is prominently discussed to be effective against obesity. Therefore, the AZD3965 and linifanib compounds are also promising drugs for future trials in obesity, T2D, or related metabolic diseases.

In contrast, none of the other candidates presented in this review is described to be potently targeted by compounds already in clinical testing. However, given the large body of literature, the ideal novel drug targets for the treatment of obesity, NAFLD, insulin resistance, and T2D are SLC13A5/NaCT and SLC16A11/MCT11. SLC13A5 gene expression was found to be associated with hepatic steatosis in humans, and the knockout mouse model was protected from obesity, fatty liver, insulin resistance, and T2D. Specific NaCT inhibition ameliorated these phenotypes, and $\mathrm{NaCT}$ is therefore an attractive novel target to investigate in clinical trials, although genome-wide association data are lacking to date. Opposite to NaCT, which is missing in GWAS, but well-characterized in terms of beneficial metabolic effects using specific inhibitory compounds, MCT11 is outstanding regarding the great number of GWAS linking SLC16A11 polymorphisms to T2D in various populations and moreover partially functional characterization. However, no specific compound has been described to date as activating MCT11 function, the modulation of the transporter that most likely has therapeutic benefit. Considering the short period of research going on for $\mathrm{NaCT}$ and MCT11, compared with the early discovery of renal inhibition of glucose transport restoring blood glucose levels in humans mediated by SGLT1 and SGLT2, or the link between brown adipose tissue thermogenesis and obesity mediated by UCP1, existing data are already promising, and continuing research will establish the novel candidates for pharmacological intervention.

The transporter modulation needed to achieve beneficial metabolic effects seems to be clear for $\mathrm{NaCT}$ and MCT11, that is, they most likely need to be inhibited and activated, respectively. Data on the involvement of other candidates in metabolic disease are very strong, for example, SLC30A8/ZnT8, but partially controversial or at least diverse data do not permit a clear conclusion as to whether an activating or inhibiting compound is more likely to improve metabolic diseases. Nevertheless, as is the problem for most of the SLCs, structural characterization is lacking and impedes the design of small-molecule compounds. Therefore, research into the three-dimensional structures of the transporters is just as important as characterization on a functional level to approach clinical trials in patients suffering from diabetes and related metabolic disease.

Finally, multiple members of the SLC13, SLC16, SLC25, and SLC30 families were linked to metabolic disease in at least one human or mouse study. Importantly, we did not evaluate the druggability of our proposed transporters, but the majority of SLCs is expected to be generally druggable. Moreover, we critically note that some of our proposed targets are only marginally linked to metabolic diseases, defined by disease patterns involved in obesity, fatty liver, insulin resistance, $\beta$-cell loss, and T2D, or other abnormalities in metabolic pathways that increase the risk of these phenotypes. However, the main purpose of this review was not only to present well-described SLC family members with an obvious role in metabolic dysfunction and multiple GWAS linking variants directly to obesity or T2D, but also to raise interest in SLC transporters, which are to date unsatisfactorily characterized and underestimated in the scientific field, but potentially also present novel targets for medical management of diseases with increasing prevalence worldwide. 


\section{Acknowledgments}

We thank Dr. Franziska W. Schwarz (www.scivisto.com) for the graphic design of the figures.

\section{Authorship Contributions}

Wrote or contributed to the writing of the manuscript: Schumann, Birkenfeld, Fromm, König, Jordan, Henke, Willmes, Bornstein.

\section{References}

Abplanalp J, Laczko E, Philp NJ, Neidhardt J, Zuercher J, Braun P, Schorderet DF, Munier FL, Verrey F, Berger W, et al. (2013) The cataract and glucosuria associated monocarboxylate transporter MCT12 is a new creatine transporter. Hum Mol Genet 22:3218-3226.

Abulizi A, Perry RJ, Camporez JPG, Jurczak MJ, Petersen KF, Aspichueta P, and Shulman GI (2017) A controlled-release mitochondrial protonophore reverses hypertriglyceridemia, nonalcoholic steatohepatitis, and diabetes in lipodystrophic mice. FASEB J 31:2916-2924.

Akanuma H, Ogawa K, Lee Y, and Akanuma Y (1981) Reduced levels of plasma 1,5-anhydroglucitol in diabetic patients. J Biochem 90:157-162.

Almeda-Valdes P, Gómez Velasco DV, Arellano Campos O, Bello-Chavolla OY, Del Rocío Sevilla-González M, Viveros Ruiz T, Martagón Rosado AJ, Bautista CJ, Muñoz Hernandez L, Cruz-Bautista I, et al. (2019) The SLC16A11 risk haplotype is associated with decreased insulin action, higher transaminases and large-size adipocytes. Eur J Endocrinol 180:99-107.

Anunciado-Koza RP, Zhang J, Ukropec J, Bajpeyi S, Koza RA, Rogers RC, Cefalu WT, Mynatt RL, and Kozak LP (2011) Inactivation of the mitochondrial carrier SLC25A25 (ATP-Mg2+/Pi transporter) reduces physical endurance and metabolic efficiency in mice. J Biol Chem 286:11659-11671.

Arsenijevic D, Onuma H, Pecqueur C, Raimbault S, Manning BS, Miroux B, Couplan E, Alves-Guerra MC, Goubern M, Surwit R, et al. (2000) Disruption of the uncoupling protein-2 gene in mice reveals a role in immunity and reactive oxygen species production. Nat Genet 26:435-439.

Balasubramaniam S, Lewis B, Greed L, Meili D, Flier A, Yamamoto R, Bilić K, Till C, and Sass JO (2016) Heterozygous monocarboxylate transporter 1 (MCT1, SLC16A1) deficiency as a cause of recurrent ketoacidosis. JIMD Rep 29:33-38.

Baumruk F, Flachs P, Horáková M, Floryk D, and Kopecký J (1999) Transgenic UCP1 in white adipocytes modulates mitochondrial membrane potential. FEBS Lett 444:206-210.

Bek-Jensen H, Fornander AM, Nilsson MA, and Tiselius HG (1996) Is citrate an inhibitor of calcium oxalate crystal growth in high concentrations of urine? Urol Res 24:67-71.

Beloueche-Babari M, Wantuch S, Casals Galobart T, Koniordou M, Parkes HG, Arunan V, Chung YL, Eykyn TR, Smith PD, and Leach MO (2017) MCT1 inhibitor AZD3965 increases mitochondrial metabolism, facilitating combination therapy and noninvasive magnetic resonance spectroscopy. Cancer Res 77:5913-5924.

Bento JL, Palmer ND, Zhong M, Roh B, Lewis JP, Wing MR, Pandya H, Freedman BI, Langefeld CD, Rich SS, et al. (2008) Heterogeneity in gene loci associated with type 2 diabetes on human chromosome 20q13.1. Genomics 92:226-234.

Berentzen T, Dalgaard LT, Petersen L, Pedersen O, and Sørensen TI (2005) Interactions between physical activity and variants of the genes encoding uncoupling proteins -2 and -3 in relation to body weight changes during a $10-y$ follow-up. Int $J$ Obes 29:93-99.

Bergeron MJ, Clémençon B, Hediger MA, and Markovich D (2013) SLC13 family of $\mathrm{Na}^{+}$-coupled di- and tri-carboxylate/sulfate transporters. Mol Aspects Med 34 $299-312$.

Birkenfeld AL, Jordan J, Dworak M, Merkel T, and Burnstock G (2019) Myocardial metabolism in heart failure: purinergic signalling and other metabolic concepts. Pharmacol Ther 194:132-144.

Birkenfeld AL, Lee HY, Guebre-Egziabher F, Alves TC, Jurczak MJ, Jornayvaz FR, Zhang D, Hsiao JJ, Martin-Montalvo A, Fischer-Rosinsky A, et al. (2011) Deletion of the mammalian INDY homolog mimics aspects of dietary restriction and protects against adiposity and insulin resistance in mice. Cell Metab 14:184-195.

Birkenfeld AL and Shulman GI (2014) Nonalcoholic fatty liver disease, hepatic in sulin resistance, and type 2 diabetes. Hepatology 59:713-723.

Boehmer C, Embark HM, Bauer A, Palmada M, Yun CH, Weinman EJ, Endou H, Cohen P, Lahme S, Bichler KH, et al. (2004) Stimulation of renal Na+ dicarboxylate cotransporter 1 by $\mathrm{Na}+/ \mathrm{H}+$ exchanger regulating factor 2 , serum and glucocorticoid inducible kinase isoforms, and protein kinase B. Biochem Biophys Res Commun 313:998-1003.

Boesgaard TW, Zilinskaite J, Vänttinen M, Laakso M, Jansson PA, Hammarstedt A Smith U, Stefan N, Fritsche A, Häring H, et al.; EUGENE 2 Consortium (2008) The common SLC30A8 Arg325Trp variant is associated with reduced first-phase in sulin release in 846 non-diabetic offspring of type 2 diabetes patients--the EUGENE2 study. Diabetologia 51:816-820.

Bola BM, Chadwick AL, Michopoulos F, Blount KG, Telfer BA, Williams KJ, Smith PD, Critchlow SE, and Stratford IJ (2014) Inhibition of monocarboxylate transporter-1 (MCT1) by AZD3965 enhances radiosensitivity by reducing lactate transport. Mol Cancer Ther 13:2805-2816.

Boss O, Samec S, Paoloni-Giacobino A, Rossier C, Dulloo A, Seydoux J, Muzzin P, and Giacobino JP (1997) Uncoupling protein-3: a new member of the mitochondrial carrier family with tissue-specific expression. FEBS Lett 408:39-42.

Bouillaud F, Alves-Guerra MC, and Ricquier D (2016) UCPs, at the interface between bioenergetics and metabolism. Biochim Biophys Acta 1863:2443-2456.

Brachs S, Winkel AF, Tang H, Birkenfeld AL, Brunner B, Jahn-Hofmann K, Margerie D, Ruetten H, Schmoll D, and Spranger J (2016) Inhibition of citrate cotransporter Slc13a5/mINDY by RNAi improves hepatic insulin sensitivity and prevents diet-induced non-alcoholic fatty liver disease in mice. Mol Metab 5: 1072-1082.

Bray GA, Blackburn GL, Ferguson JM, Greenway FL, Jain AK, Mendel CM, Mendels J, Ryan DH, Schwartz SL, Scheinbaum ML, et al. (1999) Sibutramine produces dose-related weight loss. Obes Res 7:189-198.

Brondani LA, Assmann TS, de Souza BM, Bouças AP, Canani LH, and Crispim D (2014a) Meta-analysis reveals the association of common variants in the uncoupling protein (UCP) 1-3 genes with body mass index variability. PLoS One 9: e96411.

Brondani LA, Assmann TS, Duarte GC, Gross JL, Canani LH, and Crispim D (2012) The role of the uncoupling protein 1 (UCP1) on the development of obesity and type 2 diabetes mellitus. Arq Bras Endocrinol Metabol 56:215-225.

Brondani LA, de Souza BM, Assmann TS, Bouças AP, Bauer AC, Canani LH, and Crispim D (2014b) Association of the UCP polymorphisms with susceptibility to obesity: case-control study and meta-analysis [published correction appears in Mol Biol Rep (2014) 41:6325]. Mol Biol Rep 41:5053-5067.

Bulotta A, Ludovico O, Coco A, Di Paola R, Quattrone A, Carella M, Pellegrini F, Prudente S, and Trischitta V (2005) The common -866G/A polymorphism in the promoter region of the UCP-2 gene is associated with reduced risk of type 2 diabetes in Caucasians from Italy. J Clin Endocrinol Metab 90:1176-1180.

Busiello RA, Savarese S, and Lombardi A (2015) Mitochondrial uncoupling proteins and energy metabolism. Front Physiol 6:36

Campagno RV, Severin MJ, Nosetto EC, Brandoni A, and Torres AM (2018) Renal expression and urinary excretion of $\mathrm{Na}^{+}$/dicarboxylate cotransporter 1 (NaDC1) in obstructive nephropathy: a candidate biomarker for this pathology. Pflugers Arch 470:1777-1786.

Canis M, Maurer MH, Kuschinsky W, Duembgen L, and Duelli R (2009) Increased densities of monocarboxylate transporter MCT1 after chronic hyperglycemia in rat brain. Brain Res 1257:32-39.

Carneiro L, Asrih M, Repond C, Sempoux C, Stehle JC, Leloup C, Jornayvaz FR and Pellerin L (2017) AMPK activation caused by reduced liver lactate metabolism protects against hepatic steatosis in MCT1 haploinsufficient mice. Mol Metab 6: $1625-1633$

Carneiro L and Pellerin L (2015) Monocarboxylate transporters: new players in body weight regulation. Obes Rev 16 (Suppl 1):55-66.

Cassell PG, Saker PJ, Huxtable SJ, Kousta E, Jackson AE, Hattersley AT, Frayling TM, Walker M, Kopelman PG, Ramachandran A, et al. (2000) Evidence that single nucleotide polymorphism in the uncoupling protein 3 (UCP3) gene influences fat distribution in women of European and Asian origin. Diabetologia 43: 1558-1564

Cauchi S, Del Guerra S, Choquet H, D’Aleo V, Groves CJ, Lupi R, McCarthy MI, Froguel P and Marchetti P (2010) Meta-analysis and functional effects of the SLC30A8 rs13266634 polymorphism on isolated human pancreatic islets. Mol Genet Metab 100:77-82.

César-Razquin A, Snijder B, Frappier-Brinton T, Isserlin R, Gyimesi G, Bai X, Reithmeier RA, Hepworth D, Hediger MA, Edwards AM, et al. (2015) A call for systematic research on solute carriers. Cell 162:478-487.

Chang KW, Chen HL, Chien YH, Chen TC, and Yeh CT (2011) SLC25A13 gene mutations in Taiwanese patients with non-viral hepatocellular carcinoma. $\mathrm{Mol}$ Genet Metab 103:293-296.

Chasis H, Jolliffe N, and Smith HW (1933) The action of phlorizin on the excretion of glucose, xylose, sucrose, creatinine and urea by man. J Clin Invest 12:1083-1090. Chathoth S, Ismail MH, Vatte C, Cyrus C, Al Ali Z, Ahmed KA, Acharya S, Al Barqi $\mathrm{AM}$, and Al Ali A (2018) Association of Uncoupling Protein 1 (UCP1) gene polymorphism with obesity: a case-control study. BMC Med Genet 19:203.

Chausmer AB (1998) Zinc, insulin and diabetes. J Am Coll Nutr 17:109-115.

Chen X, Tsukaguchi H, Chen XZ, Berger UV, and Hediger MA (1999) Molecular and functional analysis of SDCT2, a novel rat sodium-dependent dicarboxylate transporter. J Clin Invest 103:1159-1168.

Chen XZ, Shayakul C, Berger UV, Tian W, and Hediger MA (1998) Characterization of a rat $\mathrm{Na}$--dicarboxylate cotransporter. J Biol Chem 273:20972-20981.

Cheurfa N, Dubois-Laforgue D, Ferrarezi DA, Reis AF, Brenner GM, Bouché C, Le Feuvre C, Fumeron F, Timsit J, Marre M, et al. (2008) The common -866G $>$ A variant in the promoter of UCP2 is associated with decreased risk of coronary artery disease in type 2 diabetic men. Diabetes 57:1063-1068.

Chimienti F, Devergnas S, Favier A, and Seve M (2004) Identification and cloning of a beta-cell-specific zinc transporter, ZnT-8, localized into insulin secretory granules. Diabetes 53:2330-2337.

Chimienti F, Devergnas S, Pattou F, Schuit F, Garcia-Cuenca R, Vandewalle B, KerrConte J, Van Lommel L, Grunwald D, Favier A, et al. (2006) In vivo expression and functional characterization of the zinc transporter $\mathrm{ZnT} 8$ in glucose-induced insulin secretion. J Cell Sci 119:4199-4206.

Chung SD, Chen YK, and Lin HC (2011) Increased risk of diabetes in patients with urinary calculi: a 5-year followup study. J Urol 186:1888-1893.

Clapham JC, Arch JR, Chapman H, Haynes A, Lister C, Moore GB, Piercy V, Carter SA, Lehner I, Smith SA, et al. (2000) Mice overexpressing human uncoupling protein-3 in skeletal muscle are hyperphagic and lean. Nature 406:415-418.

Cypess AM, Lehman S, Williams G, Tal I, Rodman D, Goldfine AB, Kuo FC, Palmer EL, Tseng YH, Doria A, et al. (2009) Identification and importance of brown adipose tissue in adult humans. $N$ Engl $J$ Med 360:1509-1517.

D’Adamo M, Perego L, Cardellini M, Marini MA, Frontoni S, Andreozzi F, Sciacqua A, Lauro D, Sbraccia P, Federici M, et al. (2004) The -866A/A genotype in the promoter of the human uncoupling protein 2 gene is associated with insulin resistance and increased risk of type 2 diabetes. Diabetes 53:1905-1910.

Dalgaard LT (2011) Genetic variance in uncoupling protein 2 in relation to obesity, type 2 diabetes, and related metabolic traits: focus on the functional $-866 \mathrm{G}>\mathrm{A}$ promoter variant (rs659366). J Obes 2011:340241.

Dalgaard LT, Andersen G, Larsen LH, Sørensen TI, Andersen T, Drivsholm T, BorchJohnsen K, Fleckner J, Hansen T, Din N, et al. (2003) Mutational analysis of the UCP2 core promoter and relationships of variants with obesity. Obes Res 11: $1420-1427$ 
Dalgaard LT, Hansen T, Urhammer SA, Drivsholm T, Borch-Johnsen K, and Pedersen O (2001a) The uncoupling protein $3-55 \mathrm{C}-->\mathrm{T}$ variant is not associated with type II diabetes mellitus in Danish subjects. Diabetologia 44:1065-1067.

Dalgaard LT and Pedersen O (2001) Uncoupling proteins: functional characteristics and role in the pathogenesis of obesity and type II diabetes. Diabetologia 44: 946-965

Dalgaard LT, Sørensen TI, Drivsholm T, Borch-Johnsen K, Andersen T, Hansen T, and Pedersen O (2001b) A prevalent polymorphism in the promoter of the UCP3 gene and its relationship to body mass index and long term body weight change in the Danish population. J Clin Endocrinol Metab 86:1398-1402.

da Silva Xavier G, Bellomo EA, McGinty JA, French PM, and Rutter GA (2013) Animal models of GWAS-identified type 2 diabetes genes. J Diabetes Res 2013: 906590.

Davies MJ, D’Alessio DA, Fradkin J, Kernan WN, Mathieu C, Mingrone G, Rossing P, Tsapas A, Wexler DJ, and Buse JB (2018) Management of hyperglycemia in type 2 diabetes, 2018: a consensus report by the American Diabetes Association (ADA) and the European Association for the Study of Diabetes (EASD). Diabetes Care 41 2669-2701.

Dawson PA, Beck L, and Markovich D (2003) Hyposulfatemia, growth retardation reduced fertility, and seizures in mice lacking a functional NaSi-1 gene. Proc Natl Acad Sci USA 100:13704-13709.

Dawson PA, Choyce A, Chuang C, Whitelock J, Markovich D, and Leggatt GR (2010) Enhanced tumor growth in the NaS1 sulfate transporter null mouse. Cancer Sci 101:369-373.

Dawson PA, Gardiner B, Grimmond S, and Markovich D (2006) Transcriptional profile reveals altered hepatic lipid and cholesterol metabolism in hyposulfatemic NaS1 null mice. Physiol Genomics 26:116-124.

Dawson PA, Sim P, Simmons DG, and Markovich D (2011) Fetal loss and hyposulfataemia in pregnant NaS1 transporter null mice. J Reprod Dev 57:444-449.

Dawson PA, Steane SE, and Markovich D (2004) Behavioural abnormalities of the hyposulphataemic Nas1 knock-out mouse. Behav Brain Res 154:457-463.

Dawson PA, Steane SE, and Markovich D (2005) Impaired memory and olfactory performance in NaSi-1 sulphate transporter deficient mice. Behav Brain Res $\mathbf{1 5 9}$ $15-20$.

Derosa G and Maffioli P (2018) Ertugliflozin: a sodium-glucose cotransporter-2 (SGLT-2) inhibitor for glycemic control in type 2 diabetes. Ther Clin Risk Manag 14:1637-1640.

de Souza BM, Brondani LA, Bouças AP, Sortica DA, Kramer CK, Canani LH, Leitão CB, and Crispim D (2013) Associations between UCP1 -3826A/G, UCP2 -866G/A Ala55Val and Ins/Del, and UCP3 -55C/T polymorphisms and susceptibility to type 2 diabetes mellitus: case-control study and meta-analysis. PLoS One 8:e54259.

Dhamrait SS, Stephens JW, Cooper JA, Acharya J, Mani AR, Moore K, Miller GJ, Humphries SE, Hurel SJ, and Montgomery HE (2004) Cardiovascular risk in healthy men and markers of oxidative stress in diabetic men are associated with common variation in the gene for uncoupling protein 2. Eur Heart $J$ 25:468-475.

Ding M, Chavarro J, Olsen S, Lin Y, Ley SH, Bao W, Rawal S, Grunnet LG, Thuesen ACB, Mills JL, et al. (2018) Genetic variants of gestational diabetes mellitus: a study of 112 SNPs among 8722 women in two independent populations. Diabetologia 61:1758-1768.

Dodson G and Steiner D (1998) The role of assembly in insulin's biosynthesis. Curr Opin Struct Biol 8:189-194.

Dong F, Zhang BH, Zheng SL, Huang XX, Du XB, Zhu KH, Chen XJ, Wu J, Liu DD, Wen ZH, et al. (2018) Association between SLC30A8 rs13266634 polymorphism and risk of T2DM and IGR in Chinese population: a systematic review and metaanalysis. Front Endocrinol (Lausanne) 9:564

Drake I, Hindy G, Ericson U, and Orho-Melander M (2017) A prospective study of dietary and supplemental zinc intake and risk of type 2 diabetes depending on genetic variation in SLC30A8. Genes Nutr 12:30.

Echtay KS, Roussel D, St-Pierre J, Jekabsons MB, Cadenas S, Stuart JA, Harper JA, Roebuck SJ, Morrison A, Pickering S, et al. (2002) Superoxide activates mitochondrial uncoupling proteins. Nature 415:96-99.

Ehrenkranz JR, Lewis NG, Kahn CR, and Roth J (2005) Phlorizin: a review. Diabetes Metab Res Rev 21:31-38.

Emdin SO, Dodson GG, Cutfield JM, and Cutfield SM (1980) Role of zinc in insulin biosynthesis: some possible zinc-insulin interactions in the pancreatic B-cell. Diabetologia 19:174-182.

Enerbäck S, Jacobsson A, Simpson EM, Guerra C, Yamashita H, Harper ME, and Kozak LP (1997) Mice lacking mitochondrial uncoupling protein are coldsensitive but not obese. Nature 387:90-94.

Engelmann J, Manuwald U, Rubach C, Kugler J, Birkenfeld AL, Hanefeld M, and Rothe U (2016) Determinants of mortality in patients with type 2 diabetes: a review. Rev Endocr Metab Disord 17:129-137.

Esterbauer H, Oberkofler H, Liu YM, Breban D, Hell E, Krempler F, and Patsch W (1998) Uncoupling protein-1 mRNA expression in obese human subjects: the role of sequence variations at the uncoupling protein-1 gene locus. J Lipid Res 39:834-844.

Esterbauer H, Schneitler C, Oberkofler H, Ebenbichler C, Paulweber B, Sandhofer F Ladurner G, Hell E, Strosberg AD, Patsch JR, et al. (2001) A common polymorphism in the promoter of UCP2 is associated with decreased risk of obesity in middle-aged humans. Nat Genet 28:178-183.

Fauman EB, Rai BK, and Huang ES (2011) Structure-based druggability assessment--identifying suitable targets for small molecule therapeutics. Curr Opin Chem Biol 15:463-468.

Feldmann HM, Golozoubova V, Cannon B, and Nedergaard J (2009) UCP1 ablation induces obesity and abolishes diet-induced thermogenesis in mice exempt from thermal stress by living at thermoneutrality. Cell Metab 9:203-209.

Fioretto P, Giaccari A, and Sesti G (2015) Efficacy and safety of dapagliflozin, a sodium glucose cotransporter 2 (SGLT2) inhibitor, in diabetes mellitus. Cardiovasc Diabetol 14:142.

Fisel P, Schaeffeler E, and Schwab M (2018) Clinical and functional relevance of the monocarboxylate transporter family in disease pathophysiology and drug therapy. Clin Transl Sci 11:352-364.
Flannick J, Thorleifsson G, Beer NL, Jacobs SB, Grarup N, Burtt NP, Mahajan A, Fuchsberger $\mathrm{C}$, Atzmon $\mathrm{G}$, Benediktsson $\mathrm{R}$, et al: Go-T2D Consortium T2D-GENES Consortium (2014) Loss-of-function mutations in SLC30A8 protect against type 2 diabetes. Nat Genet 46:357-363.

Fleury C, Neverova M, Collins S, Raimbault S, Champigny O, Levi-Meyrueis C, Bouillaud F, Seldin MF, Surwit RS, Ricquier D, et al. (1997) Uncoupling protein-2: a novel gene linked to obesity and hyperinsulinemia. Nat Genet 15:269-272.

Foster MC, Leapman RD, Li MX, and Atwater I (1993) Elemental composition of secretory granules in pancreatic islets of Langerhans. Biophys J 64:525-532.

Fukumoto K, Sumida Y, Yoshida N, Sakai K, Kanemasa K, Itoh Y, Mitsufuji S, Kataoka K, and Okanoue T (2008) A case of adult-onset type II citrullinemia having a liver histology of nonalcoholic steatohepatitis (NASH). Nihon Shokakibyo Gakkai Zasshi 105:244-251.

Fukunaka A and Fujitani Y (2018) Role of zinc homeostasis in the pathogenesis of diabetes and obesity. Int $J$ Mol Sci 19:E476.

Fukuzawa T, Fukazawa M, Ueda O, Shimada H, Kito A, Kakefuda M, Kawase Y, Wada NA, Goto C, Fukushima N, et al. (2013) SGLT5 reabsorbs fructose in the kidney but its deficiency paradoxically exacerbates hepatic steatosis induced by fructose. PLoS One 8:e56681.

Gable DR, Stephens JW, Cooper JA, Miller GJ, and Humphries SE (2006) Variation in the UCP2-UCP3 gene cluster predicts the development of type 2 diabetes in healthy middle-aged men. Diabetes 55:1504-1511.

Garg VK, Gupta R, and Goyal RK (1994) Hypozincemia in diabetes mellitus. J Assoc Physicians India 42:720-721.

Gates AC, Bernal-Mizrachi C, Chinault SL, Feng C, Schneider JG, Coleman T, Malone JP, Townsend RR, Chakravarthy MV, and Semenkovich CF (2007) Respiratory uncoupling in skeletal muscle delays death and diminishes age-related disease. Cell Metab 6:497-505.

Gerber PA, Bellomo EA, Hodson DJ, Meur G, Solomou A, Mitchell RK, Hollinshead M, Chimienti F, Bosco D, Hughes SJ, et al. (2014) Hypoxia lowers SLC30A8/ZnT8 expression and free cytosolic $\mathrm{Zn} 2+$ in pancreatic beta cells. Diabetologia 57: $1635-1644$

Ghezzi C, Loo DDF, and Wright EM (2018) Physiology of renal glucose handling via SGLT1, SGLT2 and GLUT2. Diabetologia 61:2087-2097.

Ghezzi C and Wright EM (2012) Regulation of the human Na+-dependent glucose cotransporter hSGLT2. Am J Physiol Cell Physiol 303:C348-C354

Gimeno RE, Dembski M, Weng X, Deng N, Shyjan AW, Gimeno CJ, Iris F, Ellis SJ, Woolf EA, and Tartaglia LA (1997) Cloning and characterization of an uncoupling protein homolog: a potential molecular mediator of human thermogenesis. Diabetes 46:900-906.

Giralt M and Villarroya F (2017) Mitochondrial uncoupling and the regulation of glucose homeostasis. Curr Diabetes Rev 13:386-394.

Goedeke L, Perry RJ, and Shulman GI (2019) Emerging pharmacological targets for the treatment of nonalcoholic fatty liver disease, insulin resistance, and type 2 diabetes. Annu Rev Pharmacol Toxicol 59:65-87.

Goglia F and Skulachev VP (2003) A function for novel uncoupling proteins: antioxidant defense of mitochondrial matrix by translocating fatty acid peroxides from the inner to the outer membrane leaflet. FASEB J 17:1585-1591.

Golozoubova V, Hohtola E, Matthias A, Jacobsson A, Cannon B, and Nedergaard J (2001) Only UCP1 can mediate adaptive nonshivering thermogenesis in the cold FASEB J 15:2048-2050.

Gong DW, Monemdjou S, Gavrilova O, Leon LR, Marcus-Samuels B, Chou CJ, Everett C, Kozak LP, Li C, Deng C, et al. (2000) Lack of obesity and normal response to fasting and thyroid hormone in mice lacking uncoupling protein-3. $J$ Biol Chem 275:16251-16257.

González-Barroso MM, Giurgea I, Bouillaud F, Anedda A, Bellanné-Chantelot C, Hubert L, de Keyzer Y, de Lonlay P, and Ricquier D (2008) Mutations in UCP2 in congenital hyperinsulinism reveal a role for regulation of insulin secretion. PLoS One 3:e3850

Grempler R, Augustin R, Froehner S, Hildebrandt T, Simon E, Mark M, and Eickelmann P (2012) Functional characterisation of human SGLT-5 as a novel kidney-specific sodium-dependent sugar transporter. FEBS Lett 586:248-253.

Grewer C, Gameiro A, and Rauen T (2014) SLC1 glutamate transporters. Pflugers Arch 466:3-24

Halestrap AP (2012) The monocarboxylate transporter family--Structure and functional characterization. IUBMB Life 64:1-9.

Halestrap AP (2013) The SLC16 gene family - structure, role and regulation in health and disease. Mol Aspects Med 34:337-349.

Halestrap AP and Meredith D (2004) The SLC16 gene family-from monocarboxylate transporters (MCTs) to aromatic amino acid transporters and beyond. Pflugers Arch 447:619-628.

Halestrap AP and Price NT (1999) The proton-linked monocarboxylate transporter (MCT) family: structure, function and regulation. Biochem $J$ 343:281-299.

Halestrap AP and Wilson MC (2012) The monocarboxylate transporter family--role and regulation. IUBMB Life 64:109-119.

Hamada T, Kotani K, Fujiwara S, Sano Y, Domichi M, Tsuzaki K, and Sakane N (2008) The common -55 C/T polymorphism in the promoter region of the uncoupling protein 3 gene reduces prevalence of obesity and elevates serum high-density lipoprotein cholesterol levels in the general Japanese population. Metabolism 57: $410-415$.

Hamm LL (1990) Renal handling of citrate. Kidney Int 38:728-735.

Hanefeld M, Pistrosch F, Bornstein SR, and Birkenfeld AL (2016) The metabolic vascular syndrome - guide to an individualized treatment. Rev Endocr Metab Disord 17:5-17.

Hara K, Fujita H, Johnson TA, Yamauchi T, Yasuda K, Horikoshi M, Peng C, Hu C, Ma RC, Imamura M, et al.; DIAGRAM Consortium (2014) Genome-wide association study identifies three novel loci for type 2 diabetes. Hum Mol Genet 23: 239-246.

Hara T, Takeda TA, Takagishi T, Fukue K, Kambe T, and Fukada T (2017) Physiological roles of zinc transporters: molecular and genetic importance in zinc homeostasis. J Physiol Sci 67:283-301. 
Hardies K, de Kovel CG, Weckhuysen S, Asselbergh B, Geuens T, Deconinck T, Azmi A, May P, Brilstra E, Becker F, et al.; Autosomal Recessive Working Group of the EuroEPINOMICS RES Consortium (2015) Recessive mutations in SLC13A5 result in a loss of citrate transport and cause neonatal epilepsy, developmental delay and teeth hypoplasia. Brain 138:3238-3250.

Hardy AB, Wijesekara N, Genkin I, Prentice KJ, Bhattacharjee A, Kong D, Chimienti F, and Wheeler MB (2012) Effects of high-fat diet feeding on Znt8-null mice: differences between $\beta$-cell and global knockout of Znt8. Am J Physiol Endocrinol Metab 302:E1084-E1096.

Hayasaka K and Numakura C (2018) Adult-onset type II citrullinemia: current insights and therapy. Appl Clin Genet 11:163-170.

Hayasaka K, Numakura C, Toyota K, Kakizaki S, Watanabe H, Haga H, Takahashi H, Takahashi Y, Kaneko M, Yamakawa M, et al. (2014) Medium-chain triglyceride supplementation under a low-carbohydrate formula is a promising therapy for adult-onset type II citrullinemia. Mol Genet Metab Rep 1:42-50.

Hayasaka K, Numakura C, Toyota K, and Kimura T (2012) Treatment with lactose (galactose)-restricted and medium-chain triglyceride-supplemented formula for neonatal intrahepatic cholestasis caused by citrin deficiency. JIMD Rep 2:37-44.

Hayasaka K, Numakura C, Yamakawa M, Mitsui T, Watanabe H, Haga H, Yazaki M, Ohira H, Ochiai Y, Tahara T, et al. (2018) Medium-chain triglycerides supplement therapy with a low-carbohydrate formula can supply energy and enhance ammonia detoxification in the hepatocytes of patients with adult-onset type II citrullinemia. $J$ Inherit Metab Dis 41:777-784.

He W, Miao FJ, Lin DC, Schwandner RT, Wang Z, Gao J, Chen JL, Tian H, and Ling L (2004a) Citric acid cycle intermediates as ligands for orphan G-protein-coupled receptors. Nature 429:188-193.

He Y, Chen X, Yu Z, Wu D, Lv Y, Shi S, and Zhu H (2004b) Sodium dicarboxylate cotransporter-1 expression in renal tissues and its role in rat experimental nephrolithiasis. $J$ Nephrol 17:34-42.

Hebert SC, Mount DB, and Gamba G (2004) Molecular physiology of cation-coupled Cl- cotransport: the SLC12 family. Pflugers Arch 447:580-593.

Hediger MA (1994) Structure, function and evolution of solute transporters in prokaryotes and eukaryotes. J Exp Biol 196:15-49.

Hediger MA, Clémençon B, Burrier RE, and Bruford EA (2013) The ABCs of membrane transporters in health and disease (SLC series): introduction. Mol Aspects Med 34:95-107.

Hediger MA, Romero MF, Peng JB, Rolfs A, Takanaga H, and Bruford EA (2004) The ABCs of solute carriers: physiological, pathological and therapeutic implications of human membrane transport proteins introduction. Pflugers Arch 447:465-468.

Herrmann SM, Wang JG, Staessen JA, Kertmen E, Schmidt-Petersen K, Zidek W, Paul M, and Brand E (2003) Uncoupling protein 1 and 3 polymorphisms are associated with waist-to-hip ratio. J Mol Med (Berl) 81:327-332.

Hesselink MK, Mensink M, and Schrauwen P (2003) Human uncoupling protein-3 and obesity: an update. Obes Res 11:1429-1443.

Hidalgo BA, Sofer T, Qi Q, Schneiderman N, Chen YI, Kaplan RC, Avilés-Santa ML, North KE, Arnett DK, Szpiro A, et al. (2019) Associations between SLC16A11 variants and diabetes in the Hispanic Community Health Study/Study of Latinos (HCHS/SOL). Sci Rep 9:843.

Himms-Hagen J and Desautels M (1978) A mitochondrial defect in brown adipose tissue of the obese (ob/ob) mouse: reduced binding of purine nucleotides and a failure to respond to cold by an increase in binding. Biochem Biophys Res Commun 83:628-634.

Hirai T, Fukui Y, and Motojima K (2007) PPARalpha agonists positively and negatively regulate the expression of several nutrient/drug transporters in mouse small intestine. Biol Pharm Bull 30:2185-2190.

Ho HT, Ko BC, Cheung AK, Lam AK, Tam S, Chung SK, and Chung SS (2007) Generation and characterization of sodium-dicarboxylate cotransporter-deficient mice. Kidney Int 72:63-71.

Horinouchi H (2016) Anti-vascular endothelial growth factor therapies at the crossroads: linifanib for non-small cell lung cancer. Transl Lung Cancer Res $\mathbf{5}$ 78-81.

Horvath TL, Diano S, Miyamoto S, Barry S, Gatti S, Alberati D, Livak F, Lombardi A, Moreno M, Goglia F, et al. (2003) Uncoupling proteins-2 and 3 influence obesity and inflammation in transgenic mice. Int J Obes Relat Metab Disord 27:433-442

Houstis N, Rosen ED, and Lander ES (2006) Reactive oxygen species have a causal role in multiple forms of insulin resistance. Nature 440:944-948.

Hsia DS, Grove O, and Cefalu WT (2017) An update on sodium-glucose co-transporter-2 inhibitors for the treatment of diabetes mellitus. Curr Opin Endocrinol Diabetes Obes 24:73-79.

Hsu YH, Niu T, Song Y, Tinker L, Kuller LH, and Liu S (2008) Genetic variants in the UCP2-UCP3 gene cluster and risk of diabetes in the Women's Health Initiative Observational Study. Diabetes 57:1101-1107.

Huang L, Kirschke CP, Lay YA, Levy LB, Lamirande DE, and Zhang PH (2012) Znt7null mice are more susceptible to diet-induced glucose intolerance and insulin resistance. $J$ Biol Chem 287:33883-33896.

Huang L and Tepaamorndech S (2013) The SLC30 family of zinc transporters a review of current understanding of their biological and pathophysiological roles. Mol Aspects Med 34:548-560.

Huang L, Tepaamorndech S, Kirschke CP, Newman JW, Keyes WR, Pedersen TL, and Dumnil J (2018) Aberrant fatty acid metabolism in skeletal muscle contributes to insulin resistance in zinc transporter 7 (znt7)-knockout mice. J Biol Chem 293: $7549-7563$

Huang L, Yan M, and Kirschke CP (2010) Over-expression of ZnT7 increases insulin synthesis and secretion in pancreatic beta-cells by promoting insulin gene transcription. Exp Cell Res 316:2630-2643.

Huang L, Yu YY, Kirschke CP, Gertz ER, and Lloyd KK (2007) Znt7 (Slc30a7)deficient mice display reduced body zinc status and body fat accumulation. J Biol Chem 282:37053-37063.

Huard K, Brown J, Jones JC, Cabral S, Futatsugi K, Gorgoglione M, Lanba A, Vera NB, Zhu Y, Yan Q, et al. (2015) Discovery and characterization of novel inhibitors of the sodium-coupled citrate transporter (NaCT or SLC13A5). Sci Rep 5:17391.
Huard K, Gosset JR, Montgomery JI, Gilbert A, Hayward MM, Magee TV, Cabral S, Uccello DP, Bahnck K, Brown J, et al. (2016) Optimization of a dicarboxylic series for in vivo inhibition of citrate transport by the solute carrier 13 (SLC13) family. J Med Chem 59:1165-1175.

Hugo SE, Cruz-Garcia L, Karanth S, Anderson RM, Stainier DY, and Schlegel A (2012) A monocarboxylate transporter required for hepatocyte secretion of ketone bodies during fasting. Genes Dev 26:282-293.

Hui S, Ghergurovich JM, Morscher RJ, Jang C, Teng X, Lu W, Esparza LA, Reya T, Le Zhan, Yanxiang Guo J, et al. (2017) Glucose feeds the TCA cycle via circulating lactate. Nature 551:115-118.

Huizing M, Iacobazzi V, Ijlst L, Savelkoul P, Ruitenbeek W, van den Heuvel L, Indiveri C, Smeitink J, Trijbels F, Wanders R, et al. (1997) Cloning of the human carnitine-acylcarnitine carrier cDNA and identification of the molecular defect in a patient. Am J Hum Genet 61:1239-1245.

Hutton JC (1989) The insulin secretory granule. Diabetologia 32:271-281.

Indiveri $\mathrm{C}$, Iacobazzi V, Tonazzi A, Giangregorio $\mathrm{N}$, Infantino $\mathrm{V}$, Convertini $\mathrm{P}$, Console L, and Palmieri F (2011) The mitochondrial carnitine/acylcarnitine carrier: function, structure and physiopathology. Mol Aspects Med 32:223-233.

Inoue K, Fei YJ, Zhuang L, Gopal E, Miyauchi S, and Ganapathy V (2004) Functional features and genomic organization of mouse $\mathrm{NaCT}$, a sodium-coupled transporter for tricarboxylic acid cycle intermediates. Biochem $J$ 378:949-957.

Inoue K, Zhuang L, and Ganapathy V (2002a) Human Na+-coupled citrate transporter: primary structure, genomic organization, and transport function. Biochem Biophys Res Commun 299:465-471.

Inoue K, Zhuang L, Maddox DM, Smith SB, and Ganapathy V (2002b) Structure, function, and expression pattern of a novel sodium-coupled citrate transporter (NaCT) cloned from mammalian brain. J Biol Chem 277:39469-39476.

James WP, Astrup A, Finer N, Hilsted J, Kopelman P, Rössner S, Saris WH, and Van Gaal LF (2000) Effect of sibutramine on weight maintenance after weight loss: a randomised trial. STORM Study Group. Sibutramine Trial of Obesity Reduction and Maintenance. Lancet 356:2119-2125.

Jayawardena R, Ranasinghe P, Galappatthy P, Malkanthi R, Constantine G, and Katulanda P (2012) Effects of zinc supplementation on diabetes mellitus: a systematic review and meta-analysis. Diabetol Metab Syndr 4:13.

Ji Q, Ikegami H, Fujisawa T, Kawabata Y, Ono M, Nishino M, Ohishi M, Katsuya T, Rakugi H, and Ogihara T (2004) A common polymorphism of uncoupling protein 2 gene is associated with hypertension. J Hypertens 22:97-102.

Jia JJ, Zhang X, Ge CR, and Jois M (2009) The polymorphisms of UCP2 and UCP3 genes associated with fat metabolism, obesity and diabetes. Obes Rev 10.519-526.

Johnson RJ, Segal MS, Sautin Y, Nakagawa T, Feig DI, Kang DH, Gersch MS, Benner S, and Sánchez-Lozada LG (2007) Potential role of sugar (fructose) in the epidemic of hypertension, obesity and the metabolic syndrome, diabetes, kidney disease, and cardiovascular disease. Am J Clin Nutr 86:899-906.

Jones RS and Morris ME (2016) Monocarboxylate transporters: therapeutic targets and prognostic factors in disease. Clin Pharmacol Ther 100:454-463.

Ju W, Eichinger F, Bitzer M, Oh J, McWeeney S, Berthier CC, Shedden K, Cohen CD, Henger A, Krick S, et al. (2009) Renal gene and protein expression signatures for prediction of kidney disease progression. Am J Pathol 174:2073-2085.

Jun HS, Kim IK, Lee HJ, Lee HJ, Kang JH, Kim JR, Shin HD, and Song J (2009) Effects of UCP2 and UCP3 variants on the manifestation of overweight in Korean children. Obesity (Silver Spring) 17:355-362.

Jurczak MJ, Lee HY, Birkenfeld AL, Jornayvaz FR, Frederick DW, Pongratz RL, Zhao X, Moeckel GW, Samuel VT, Whaley JM, et al. (2011) SGLT2 deletion improves glucose homeostasis and preserves pancreatic beta-cell function. Diabetes 60:890-898.

Jurczak MJ, Saini S, Ioja S, Costa DK, Udeh N, Zhao X, Whaley JM, and Kibbey RG (2018) SGLT2 knockout prevents hyperglycemia and is associated with reduced pancreatic $\beta$-cell death in genetically obese mice. Islets 10:181-189.

Kahl S, Gancheva S, Strassburger K, Herder C, Manchann J, Katsuyama H, Kabisch S, Henkel E, Kopf S, Kantartzis K, et al. (2019) 133-OR: effects of empagliflozin on liver fat content in type 2 diabetes: the EMLIFA001 trial. Diabetes 68:133.

Kahn BB, Shulman GI, DeFronzo RA, Cushman SW, and Rossetti L (1991) Normalization of blood glucose in diabetic rats with phlorizin treatment reverses insulin-resistant glucose transport in adipose cells without restoring glucose transporter gene expression. J Clin Invest 87:561-570.

Kajimura S and Saito M (2014) A new era in brown adipose tissue biology: molecular control of brown fat development and energy homeostasis. Annu Rev Physiol $\mathbf{7 6}$ $225-249$

Kasahara M, Maeda M, Hayashi S, Mori Y, and Abe T (2001) A missense mutation in the $\mathrm{Na}(+)$ /glucose cotransporter gene SGLT1 in a patient with congenital glucosegalactose malabsorption: normal trafficking but inactivation of the mutant protein. Biochim Biophys Acta 1536:141-147.

Keele GR, Prokop JW, He H, Holl K, Littrell J, Deal A, Francic S, Cui L, Gatti DM, Broman KW, et al. (2018) Genetic fine-mapping and identification of candidate genes and variants for adiposity traits in outbred rats. Obesity (Silver Spring) 26 213-222.

Kiefer FW (2017) The significance of beige and brown fat in humans. Endocr Connect 6:R70-R79.

Kim I, Kang ES, Yim YS, Ko SJ, Jeong SH, Rim JH, Kim YS, Ahn CW, Cha BS, Lee HC, et al. (2011) A low-risk ZnT-8 allele (W325) for post-transplantation diabetes mellitus is protective against cyclosporin A-induced impairment of insulin secretion. Pharmacogenomics J 11:191-198.

Kimm SY, Glynn NW, Aston CE, Damcott CM, Poehlman ET, Daniels SR, and Ferrell RE (2002) Racial differences in the relation between uncoupling protein genes and resting energy expenditure. Am J Clin Nutr 75:714-719.

Kirschke CP and Huang L (2003) ZnT7, a novel mammalian zinc transporter, accumulates zinc in the Golgi apparatus. J Biol Chem 278:4096-4102.

Kleeman CR, Coburn JW, Brickman AS, Lee DB, Narins RG, and Ehrlich RM (1980) Kidney stones. West $J$ Med 132:313-332.

Kleiner S, Gomez D, Megra B, Na E, Bhavsar R, Cavino K, Xin Y, Rojas J, Dominguez-Gutierrez G, Zambrowicz B, et al. (2018) Mice harboring the human 
SLC30A8 R138X loss-of-function mutation have increased insulin secretory capacity. Proc Natl Acad Sci USA 115:E7642-E7649.

Klotz J, Porter BE, Colas C, Schlessinger A, and Pajor AM (2016) Mutations in the $\mathrm{Na}(+)$ /citrate cotransporter NaCT (SLC13A5) in pediatric patients with epilepsy and developmental delay. Mol Med 22:310-321.

Kobayashi K, Sinasac DS, Iijima M, Boright AP, Begum L, Lee JR, Yasuda T, Ikeda S, Hirano R, Terazono H, et al. (1999) The gene mutated in adult-onset type II citrullinaemia encodes a putative mitochondrial carrier protein. Nat Genet 22: 159-163.

Kolz M, Johnson T, Sanna S, Teumer A, Vitart V, Perola M, Mangino M, Albrecht E, Wallace C, Farrall M, et al.; EUROSPAN Consortium; ENGAGE Consortium; PROCARDIS Consortium; KORA Study; WTCCC (2009) Meta-analysis of 28,141 individuals identifies common variants within five new loci that influence uric acid concentrations. PLoS Genet 5:e1000504.

Komatsu M, Yazaki M, Tanaka N, Sano K, Hashimoto E, Takei Y, Song YZ, Tanaka E, Kiyosawa K, Saheki T, et al. (2008) Citrin deficiency as a cause of chronic liver disorder mimicking non-alcoholic fatty liver disease. J Hepatol 49:810-820.

König B, Koch A, Giggel K, Dordschbal B, Eder K, and Stangl GI (2008) Monocarboxylate transporter (MCT)-1 is up-regulated by PPARalpha. Biochim Biophys Acta 1780:899-904.

Kopecky J, Clarke G, Enerbäck S, Spiegelman B, and Kozak LP (1995) Expression of the mitochondrial uncoupling protein gene from the aP2 gene promoter prevents genetic obesity. J Clin Invest 96:2914-2923.

Kopecký J, Hodný Z, Rossmeisl M, Syrový I, and Kozak LP (1996a) Reduction of dietary obesity in aP2-Ucp transgenic mice: physiology and adipose tissue distribution. Am J Physiol 270:E768-E775.

Kopecký J, Rossmeisl M, Hodný Z, Syrový I, Horáková M, and Kolárová P (1996b) Reduction of dietary obesity in aP2-Ucp transgenic mice: mechanism and adipose tissue morphology. Am J Physiol 270:E776-E786.

Köttgen A, Albrecht E, Teumer A, Vitart V, Krumsiek J, Hundertmark C, Pistis G, Ruggiero D, O'Seaghdha CM, Haller T, et al.; LifeLines Cohort Study; CARDIoGRAM Consortium; DIAGRAM Consortium; ICBP Consortium; MAGIC Consortium (2013) Genome-wide association analyses identify 18 new loci associated with serum urate concentrations. Nat Genet 45:145-154.

Kring SI, Larsen LH, Holst C, Toubro S, Hansen T, Astrup A, Pedersen O, and Sørensen TI (2008) Genotype-phenotype associations in obesity dependent on definition of the obesity phenotype. Obes Facts 1:138-145

Lake AD, Novak P, Shipkova P, Aranibar N, Robertson DG, Reily MD, LehmanMcKeeman LD, Vaillancourt RR, and Cherrington NJ (2015) Branched chain amino acid metabolism profiles in progressive human nonalcoholic fatty liver disease. Amino Acids 47:603-615.

Langin D (2003) The role of uncoupling protein 2 in the development of type 2 diabetes. Drugs Today (Barc) 39:287-295.

Lara-Riegos JC, Ortiz-López MG, Peña-Espinoza BI, Montúfar-Robles I, Peña-Rico MA, Sánchez-Pozos K, Granados-Silvestre MA, and Menjivar M (2015) Diabetes susceptibility in Mayas: evidence for the involvement of polymorphisms in HHEX, HNF4 $\alpha$, KCNJ11, PPAR $\gamma$, CDKN2A/2B, SLC30A8, CDC123/CAMK1D, TCF7L2, ABCA1 and SLC16A11 genes. Gene 565:68-75.

Leitner BP, Huang S, Brychta RJ, Duckworth CJ, Baskin AS, McGehee S, Tal I, Dieckmann W, Gupta G, Kolodny GM, et al. (2017) Mapping of human brown adipose tissue in lean and obese young men. Proc Natl Acad Sci USA 114: 8649-8654.

Lemaire K, Ravier MA, Schraenen A, Creemers JW, Van de Plas R, Granvik M, Van Lommel L, Waelkens E, Chimienti F, Rutter GA, et al. (2009) Insulin crystallization depends on zinc transporter ZnT8 expression, but is not required for normal glucose homeostasis in mice. Proc Natl Acad Sci USA 106:14872-14877.

Lengacher S, Nehiri-Sitayeb T, Steiner N, Carneiro L, Favrod C, Preitner F, Thorens B, Stehle JC, Dix L, Pralong F, et al. (2013) Resistance to diet-induced obesity and associated metabolic perturbations in haploinsufficient monocarboxylate transporter 1 mice. PLoS One 8:e82505.

Li B, Nolte LA, Ju JS, Han DH, Coleman T, Holloszy JO, and Semenkovich CF (2000) Skeletal muscle respiratory uncoupling prevents diet-induced obesity and insulin resistance in mice. Nat Med 6:1115-1120.

Li L, Bai S, and Sheline CT (2017a) hZnT8 (Slc30a8) transgenic mice that overexpress the R325W polymorph have reduced islet $\mathrm{Zn} 2+$ and proinsulin levels, increased glucose tolerance after a high-fat diet, and altered levels of pancreatic zinc binding proteins. Diabetes 66:551-559.

Li L, Li H, Garzel B, Yang H, Sueyoshi T, Li Q, Shu Y, Zhang J, Hu B, Heyward S, et al. (2015) SLC13A5 is a novel transcriptional target of the pregnane X receptor and sensitizes drug-induced steatosis in human liver. Mol Pharmacol 87: 674-682.

Li M, Maruthur NM, Loomis SJ, Pietzner M, North KE, Mei H, Morrison AC, Friedrich N, Pankow JS, Nauck M, et al. (2017b) Genome-wide association study of 1,5anhydroglucitol identifies novel genetic loci linked to glucose metabolism. Sci Rep 7:2812.

Li YY, Lu XZ, Wang H, Yang XX, Geng HY, Gong G, Zhan YY, Kim HJ, and Yang ZJ (2018) Solute carrier family 30 member 8 gene $807 \mathrm{C} / \mathrm{T}$ polymorphism and type 2 diabetes mellitus in the Chinese population: a meta-analysis including 6,942 subjects. Front Endocrinol (Lausanne) 9:263.

Lin L, Yee SW, Kim RB, and Giacomini KM (2015) SLC transporters as therapeutic targets: emerging opportunities. Nat Rev Drug Discov 14:543-560.

Lin Z, Wang Y, Zhang B, and Jin Z (2018) Association of type 2 diabetes susceptible genes GCKR, SLC30A8, and FTO polymorphisms with gestational diabetes mellitus risk: a meta-analysis. Endocrine 62:34-45.

Lindholm E, Klannemark M, Agardh E, Groop L, and Agardh CD (2004) Putative role of polymorphisms in UCP1-3 genes for diabetic nephropathy. J Diabetes Complications 18:103-107.

Lindquist B and Meeuwisse GW (1962) Chronic diarrhoea caused by monosaccharide malabsorption. Acta Paediatr (Stockh) 51:674-685.

Liu J, Li J, Li WJ, and Wang CM (2013) The role of uncoupling proteins in diabetes mellitus. J Diabetes Res 2013:585897.
Liu J, Wang L, Qian Y, Dai J, Shen C, Jin G, Hu Z, and Shen H (2018) Association of 48 type 2 diabetes susceptibility loci with fasting plasma glucose and lipid levels in Chinese Hans. Diabetes Res Clin Pract 139:114-121.

Liu YJ, Liu PY, Long J, Lu Y, Elze L, Recker RR, and Deng HW (2005) Linkage and association analyses of the UCP3 gene with obesity phenotypes in Caucasian families. Physiol Genomics 22:197-203.

Lokman FE, Seman NA, Ismail AA, Yaacob NA, Mustafa N, Khir AS, Hussein Z and Wan Mohamud WN (2011) Gene expression profiling in ethnic Malays with type 2 diabetes mellitus, with and without diabetic nephropathy. J Nephrol 24: $778-789$.

Long T, Hicks M, Yu HC, Biggs WH, Kirkness EF, Menni C, Zierer J, Small KS, Mangino M, Messier H, et al. (2017) Whole-genome sequencing identifies commonto-rare variants associated with human blood metabolites. Nat Genet 49:568-578.

Lowell BB (1999) Uncoupling protein-3 (UCP3): a mitochondrial carrier in search of a function. Int J Obes Relat Metab Disord 23 (Suppl 6):S43-S45.

Lowell BB, S-Susulic V, Hamann A, Lawitts JA, Himms-Hagen J, Boyer BB, Kozak LP, and Flier JS (1993) Development of obesity in transgenic mice after genetic ablation of brown adipose tissue. Nature 366:740-742.

Lyssenko V, Almgren P, Anevski D, Orho-Melander M, Sjögren M, Saloranta C, Tuomi T, and Groop L; Botnia Study Group (2005) Genetic prediction of future type 2 diabetes. PLoS Med 2:e345.

Mahmood E, Jeganathan J, Feng R, Saraf M, Khabbaz K, Mahmood F, Venkatachalam S, Liu D, Chu L, Parikh SM, et al. (2019) Decreased PGC-1 $\alpha$ postcardiopulmonary bypass leads to impaired oxidative stress in diabetic patients. Ann Thorac Surg 107:467-476.

Mailloux RJ and Harper ME (2011) Uncoupling proteins and the control of mitochondrial reactive oxygen species production. Free Radic Biol Med 51:1106-1115.

Mak IW, Evaniew N, and Ghert M (2014) Lost in translation: animal models and clinical trials in cancer treatment. Am J Transl Res 6:114-118.

Maret W (2017) Zinc in pancreatic islet biology, insulin sensitivity, and diabetes. Prev Nutr Food Sci 22:1-8.

Markovich D (2001) Physiological roles and regulation of mammalian sulfate transporters. Physiol Rev 81:1499-1533.

Markovich D and Murer H (2004) The SLC13 gene family of sodium sulphate/carboxylate cotransporters. Pflugers Arch 447:594-602.

Martín MG, Turk E, Lostao MP, Kerner C, and Wright EM (1996) Defects in Na+/ glucose cotransporter (SGLT1) trafficking and function cause glucose-galactose malabsorption. Nat Genet 12:216-220.

Maruthur NM, Clark JM, Fu M, Linda Kao WH, and Shuldiner AR (2015) Effect of zinc supplementation on insulin secretion: interaction between zinc and SLC30A8 genotype in Old Order Amish. Diabetologia 58:295-303.

Mathieu C, Dandona P, Gillard P, Senior P, Hasslacher C, Araki E, Lind M, Bain SC, Jabbour S, Arya N, et al.; DEPICT-2 Investigators (2018) Efficacy and safety of dapagliflozin in patients with inadequately controlled type 1 diabetes (the DEPICT-2 study): 24-week results from a randomized controlled trial. Diabetes Care 41:1938-1946.

McCrimmon RJ and Henry RR (2018) SGLT inhibitor adjunct therapy in type 1 diabetes. Diabetologia 61:2126-2133.

McKeegan EM, Ansell PJ, Davis G, Chan S, Chandran RK, Gawel SH, Dowell BL, Bhathena A, Chakravartty A, McKee MD, et al. (2015) Plasma biomarker signature associated with improved survival in advanced non-small cell lung cancer patients on linifanib. Lung Cancer 90:296-301.

Meirhaeghe A, Amouyel P, Helbecque N, Cottel D, Otabe S, Froguel P, and Vasseur F (2000) An uncoupling protein 3 gene polymorphism associated with a lower risk of developing type II diabetes and with atherogenic lipid profile in a French cohort. Diabetologia 43:1424-1428.

Meissner T, Otonkoski T, Feneberg R, Beinbrech B, Apostolidou S, Sipilä I, Schaefer F, and Mayatepek E (2001) Exercise induced hypoglycaemic hyperinsulinism. Arch Dis Child 84:254-257.

Merriman C, Huang Q, Rutter GA, and Fu D (2016) Lipid-tuned zinc transport activity of human ZnT8 protein correlates with risk for type-2 diabetes. J Biol Chem 291:26950-26957.

Metz L, Mercier J, Tremblay A, Almeras N, and Joanisse DR (2008) Effect of weight loss on lactate transporter expression in skeletal muscle of obese subjects. $J A p p l$ Physiol (1985) 104:633-638.

Mitchell RK, Hu M, Chabosseau PL, Cane MC, Meur G, Bellomo EA, Carzaniga R, Collinson LM, Li WH, Hodson DJ, et al. (2016) Molecular genetic regulation of Slc30a8/ZnT8 reveals a positive association with glucose tolerance. Mol Endocrinol 30:77-91.

Moore GB, Himms-Hagen J, Harper ME, and Clapham JC (2001) Overexpression of UCP-3 in skeletal muscle of mice results in increased expression of mitochondrial thioesterase mRNA. Biochem Biophys Res Commun 283:785-790.

Morris AP (2018) Progress in defining the genetic contribution to type 2 diabetes susceptibility. Curr Opin Genet Dev 50:41-51.

Nakayama A, Matsuo $H$, Shimizu T, Ogata $H$, Takada $Y$, Nakashima $H$, Nakamura T, Shimizu S, Chiba T, Sakiyama M, et al. (2013) Common missense variant of monocarboxylate transporter 9 (MCT9/SLC16A9) gene is associated with renal overload gout, but not with all gout susceptibility. Hum Cell $\mathbf{2 6}$ $133-136$

Nanayakkara S, Senevirathna ST, Abeysekera T, Chandrajith R, Ratnatunga N, Gunarathne ED, Yan J, Hitomi T, Muso E, Komiya T, et al. (2014) An integrative study of the genetic, social and environmental determinants of chronic kidney disease characterized by tubulointerstitial damages in the North Central Region of Sri Lanka. J Occup Health 56:28-38.

Neal B, Perkovic V, Mahaffey KW, de Zeeuw D, Fulcher G, Erondu N, Shaw W, Law G, Desai M, and Matthews DR; CANVAS Program Collaborative Group (2017) Canagliflozin and cardiovascular and renal events in type 2 diabetes. N Engl J Med 377:644-657.

Nedergaard J, Bengtsson T, and Cannon B (2007) Unexpected evidence for active brown adipose tissue in adult humans. Am J Physiol Endocrinol Metab 293: E444-E452. 
Nedergaard J, Golozoubova V, Matthias A, Asadi A, Jacobsson A, and Cannon B (2001) UCP1: the only protein able to mediate adaptive non-shivering thermogenesis and metabolic inefficiency. Biochim Biophys Acta 1504:82-106.

Neretti N, Wang PY, Brodsky AS, Nyguyen HH, White KP, Rogina B, and Helfand SL (2009) Long-lived Indy induces reduced mitochondrial reactive oxygen species production and oxidative damage. Proc Natl Acad Sci USA 106:2277-2282.

Neuschäfer-Rube F, Lieske S, Kuna M, Henkel J, Perry RJ, Erion DM, Pesta D, Willmes DM, Brachs S, von Loeffelholz C, et al. (2014) The mammalian INDY homolog is induced by CREB in a rat model of type 2 diabetes. Diabetes 63: 1048-1057.

Nicar MJ, Skurla C, Sakhaee K, and Pak CY (1983) Low urinary citrate excretion in nephrolithiasis. Urology 21:8-14.

Nicholls DG, Bernson VS, and Heaton GM (1978) The identification of the component in the inner membrane of brown adipose tissue mitochondria responsible for regulating energy dissipation. Experientia Suppl 32:89-93.

Nicholls DG and Locke RM (1984) Thermogenic mechanisms in brown fat. Physiol Rev 64:1-64

Nicoletti CF, de Oliveira AP, Brochado MJ, de Oliveira BP, Pinhel MA, Marchini JS, dos Santos JE, Salgado Junior W, Silva Junior WA, and Nonino CB (2016) UCP1 $-3826 \mathrm{~A}>\mathrm{G}$ polymorphism affects weight, fat mass, and risk of type 2 diabetes mellitus in grade III obese patients. Nutrition 32:83-87.

Nicolson TJ, Bellomo EA, Wijesekara N, Loder MK, Baldwin JM, Gyulkhandanyan AV, Koshkin V, Tarasov AI, Carzaniga R, Kronenberger K, et al. (2009) Insulin storage and glucose homeostasis in mice null for the granule zinc transporter $\mathrm{ZnT} 8$ and studies of the type 2 diabetes-associated variants. Diabetes $\mathbf{5 8}$ 2070-2083

Noh H, Paik HY, Kim J, and Chung J (2014) The changes of zinc transporter ZnT gene expression in response to zinc supplementation in obese women. Biol Trace Elem Res 162:38-45.

Nuttall FQ (1965) Metabolic acidosis--diabetic Arch Intern Med 116:709-716.

Ochoa MC, Santos JL, Azcona C, Moreno-Aliaga MJ, Martínez-González MA, Martínez JA, and Marti A; GENOI Members (2007) Association between obesity and insulin resistance with UCP2-UCP3 gene variants in Spanish children and adolescents. Mol Genet Metab 92:351-358.

Ohura T, Kobayashi K, Tazawa Y, Nishi I, Abukawa D, Sakamoto O, Iinuma K, and Saheki T (2001) Neonatal presentation of adult-onset type II citrullinemia. Hum Genet 108:87-90.

Okamoto N, Aruga S, Matsuzaki S, Takahashi S, Matsushita K, and Kitamura T (2007) Associations between renal sodium-citrate cotransporter (hNaDC-1) gene polymorphism and urinary citrate excretion in recurrent renal calcium stone formers and normal controls. Int J Urol 14:344-349.

O'Neil PM, Birkenfeld AL, McGowan B, Mosenzon O, Pedersen SD, Wharton S, Carson CG, Jepsen CH, Kabisch M, and Wilding JPH (2018) Efficacy and safety of semaglutide compared with liraglutide and placebo for weight loss in patients with obesity: a randomised, double-blind, placebo and active controlled, dose-ranging, phase 2 trial. Lancet 392:637-649.

Ost M, Keipert S, and Klaus S (2017) Targeted mitochondrial uncoupling beyond UCP1 - The fine line between death and metabolic health. Biochimie 134:77-85.

Otabe S, Clement K, Dina C, Pelloux V, Guy-Grand B, Froguel P, and Vasseur F (2000) A genetic variation in the $5^{\prime}$ flanking region of the UCP3 gene is associated with body mass index in humans in interaction with physical activity. Diabetologia 43:245-249.

Otonkoski T, Jiao H, Kaminen-Ahola N, Tapia-Paez I, Ullah MS, Parton LE, Schuit F, Quintens R, Sipilä I, Mayatepek E, et al. (2007) Physical exercise-induced hypoglycemia caused by failed silencing of monocarboxylate transporter 1 in pancreatic beta cells. Am J Hum Genet 81:467-474.

Otonkoski T, Kaminen N, Ustinov J, Lapatto R, Meissner T, Mayatepek E, Kere J, and Sipilä I (2003) Physical exercise-induced hyperinsulinemic hypoglycemia is an autosomal-dominant trait characterized by abnormal pyruvate-induced insulin release. Diabetes 52:199-204.

Pajor AM (2006) Molecular properties of the SLC13 family of dicarboxylate and sulfate transporters. Pflugers Arch 451:597-605.

Pajor AM (2014) Sodium-coupled dicarboxylate and citrate transporters from the SLC13 family. Pflugers Arch 466:119-130.

Pajor AM, de Oliveira CA, Song K, Huard K, Shanmugasundaram V, and Erion DM (2016) Molecular basis for inhibition of the Na+/citrate transporter NaCT (SLC13A5) by dicarboxylate inhibitors. Mol Pharmacol 90:755-765.

Pajor AM and Randolph KM (2007) Inhibition of the Na+/dicarboxylate cotransporter by anthranilic acid derivatives. Mol Pharmacol 72:1330-1336.

Pajor AM and Sun NN (2010) Single nucleotide polymorphisms in the human Na+dicarboxylate cotransporter affect transport activity and protein expression. Am J Physiol Renal Physiol 299:F704-F711.

Palmer BR, Devereaux CL, Dhamrait SS, Mocatta TJ, Pilbrow AP, Frampton CM Skelton L, Yandle TG, Winterbourn CC, Richards AM, et al (2009) The common G866A polymorphism of the UCP2 gene and survival in diabetic patients following myocardial infarction. Cardiovasc Diabetol 8:31.

Palmieri F (2004) The mitochondrial transporter family (SLC25): physiological and pathological implications. Pflugers Arch 447:689-709.

Palmieri F (2013) The mitochondrial transporter family SLC25: identification, properties and physiopathology. Mol Aspects Med 34:465-484.

Palmieri F and Monné M (2016) Discoveries, metabolic roles and diseases of mitochondrial carriers: a review. Biochim Biophys Acta 1863:2362-2378.

Palmiter RD, Cole TB, Quaife CJ, and Findley SD (1996) ZnT-3, a putative transporter of zinc into synaptic vesicles. Proc Natl Acad Sci USA 93:14934-14939.

Palmiter RD and Huang L (2004) Efflux and compartmentalization of zinc by members of the SLC30 family of solute carriers. Pflugers Arch 447:744-751.

Parascandola J (1974) Dinitrophenol and bioenergetics: an historical perspective. Mol Cell Biochem 5:69-77.

Parrinello CM and Selvin E (2014) Beyond HbA1c and glucose: the role of nontraditional glycemic markers in diabetes diagnosis, prognosis, and management. Curr Diab Rep 14:548
Pascual-Gamarra JM, Salazar-Tortosa D, Martinez-Tellez B, Labayen I, Rupérez AI, Censi L, Manios Y, Nova E, Gesteiro E, Moreno LA, et al, (2019) Association between UCP1, UCP2, and UCP3 gene polymorphisms with markers of adiposity in European adolescents: the HELENA study. Pediatr Obes 14:e12504.

Perel P, Roberts I, Sena E, Wheble P, Briscoe C, Sandercock P, Macleod M, Mignini LE, Jayaram P, and Khan KS (2007) Comparison of treatment effects between animal experiments and clinical trials: systematic review. BMJ 334:197.

Perez-Becerril C, Morris AG, Mortimer A, McKenna PJ, and de Belleroche J (2014) Allelic variants in the zinc transporter-3 gene, SLC30A3, a candidate gene identified from gene expression studies, show gender-specific association with schizophrenia. Eur Psychiatry 29:172-178.

Perland E and Fredriksson R (2017) Classification systems of secondary active transporters. Trends Pharmacol Sci 38:305-315.

Perry RJ, Zhang D, Zhang XM, Boyer JL, and Shulman GI (2015) Controlled-release mitochondrial protonophore reverses diabetes and steatohepatitis in rats. Science 347:1253-1256.

Pesta DH, Perry RJ, Guebre-Egziabher F, Zhang D, Jurczak M, Fischer-Rosinsky A Daniels MA, Willmes DM, Bhanot S, Bornstein SR, et al. (2015) Prevention of dietinduced hepatic steatosis and hepatic insulin resistance by second generation antisense oligonucleotides targeted to the longevity gene mIndy (Slc13a5). Aging (Albany NY) 7:1086-1093.

Petersen MC, Madiraju AK, Gassaway BM, Marcel M, Nasiri AR, Butrico G, Marcucci MJ, Zhang D, Abulizi A, Zhang XM, et al. (2016) Insulin receptor Thr1160 phosphorylation mediates lipid-induced hepatic insulin resistance. J Clin Invest 126:4361-4371.

Pi J, Bai Y, Daniel KW, Liu D, Lyght O, Edelstein D, Brownlee M, Corkey BE, and Collins S (2009) Persistent oxidative stress due to absence of uncoupling protein 2 associated with impaired pancreatic beta-cell function. Endocrinology 150:3040-3048.

Pierre K, Parent A, Jayet PY, Halestrap AP, Scherrer U, and Pellerin L (2007) Enhanced expression of three monocarboxylate transporter isoforms in the brain of obese mice. J Physiol 583:469-486.

Pitkänen E (1996) Mannose, mannitol, fructose and 1,5-anhydroglucitol concentrations measured by gas chromatography/mass spectrometry in blood plasma of diabetic patients. Clin Chim Acta 251:91-103.

Pitkänen OM, Vanhanen H, and Pitkänen E (1999) Metabolic syndrome is associated with changes in D-mannose metabolism. Scand J Clin Lab Invest 59:607-612.

Plengvidhya N, Chanprasert C, Chongjaroen N, Yenchitsomanus PT, Homsanit M, and Tangjittipokin W (2018) Impact of KCNQ1, CDKN2A/2B, CDKAL1, HHEX, MTNR1B, SLC30A8, TCF7L2, and UBE2E2 on risk of developing type 2 diabetes in Thai population. BMC Med Genet 19:93.

Polański R, Hodgkinson CL, Fusi A, Nonaka D, Priest L, Kelly P, Trapani F, Bishop PW, White A, Critchlow SE, et al. (2014) Activity of the monocarboxylate transporter 1 inhibitor AZD3965 in small cell lung cancer. Clin Cancer Res 20:926-937. Pound LD, Sarkar SA, Benninger RK, Wang Y, Suwanichkul A, Shadoan MK, Printz RL, Oeser JK, Lee CE, Piston DW, et al. (2009) Deletion of the mouse Slc30a8 gene encoding zinc transporter-8 results in impaired insulin secretion. Biochem J 421: 371-376.

Pound LD, Sarkar SA, Ustione A, Dadi PK, Shadoan MK, Lee CE, Walters JA, Shiota M, McGuinness OP, Jacobson DA, et al. (2012) The physiological effects of deleting the mouse SLC30A8 gene encoding zinc transporter-8 are influenced by gender and genetic background. PLoS One 7:e40972.

Powell DR, DaCosta CM, Gay J, Ding ZM, Smith M, Greer J, Doree D, Jeter-Jones S, Mseeh F, Rodriguez LA, et al. (2013) Improved glycemic control in mice lacking Sglt1 and Sglt2. Am J Physiol Endocrinol Metab 304:E117-E130.

Pramod AB, Foster J, Carvelli L, and Henry LK (2013) SLC6 transporters: structure, function, regulation, disease association and therapeutics. Mol Aspects Med 34 197-219.

Pullen TJ, Sylow L, Sun G, Halestrap AP, Richter EA, and Rutter GA (2012) Overexpression of monocarboxylate transporter-1 (SLC16A1) in mouse pancreatic $\beta$-cells leads to relative hyperinsulinism during exercise. Diabetes 61:1719-1725. Ramaswamy K and Shah O (2014) Metabolic syndrome and nephrolithiasis. Trans Androl Urol 3:285-295.

Rask-Andersen M, Almén MS, and Schiöth HB (2011) Trends in the exploitation of novel drug targets. Nat Rev Drug Discov 10:579-590.

Rask-Andersen M, Masuram S, Fredriksson R, and Schiöth HB (2013) Solute carriers as drug targets: current use, clinical trials and prospective. Mol Aspects Med 34 $702-710$

Rogers RP and Rogina B (2014) Increased mitochondrial biogenesis preserves in testinal stem cell homeostasis and contributes to longevity in Indy mutant flies. Aging (Albany NY) 6:335-350.

Rogina B (2017) INDY-a new link to metabolic regulation in animals and humans. Front Genet 8:66.

Rogina B, Reenan RA, Nilsen SP, and Helfand SL (2000) Extended life-span conferred by cotransporter gene mutations in Drosophila. Science 290:2137-2140.

Rosenstock J, Marquard J, Laffel LM, Neubacher D, Kaspers S, Cherney DZ, Zinman B, Skyler JS, George J, Soleymanlou N, et al. (2018) Empagliflozin as adjunctive to insulin therapy in type 1 diabetes: the EASE trials. Diabetes Care 41:2560-2569.

Rosenthal EA, Ranchalis J, Crosslin DR, Burt A, Brunzell JD, Motulsky AG, Nickerson DA, Wijsman EM, and Jarvik GP; NHLBI GO Exome Sequencing Project (2013) Joint linkage and association analysis with exome sequence data implicates SLC25A40 in hypertriglyceridemia. Am J Hum Genet 93:1035-1045.

Rossetti L, Smith D, Shulman GI, Papachristou D, and DeFronzo RA (1987) Correction of hyperglycemia with phlorizin normalizes tissue sensitivity to insulin in diabetic rats. J Clin Invest 79:1510-1515.

Rothwell NJ and Stock MJ (1979) A role for brown adipose tissue in diet-induced thermogenesis. Nature 281:31-35.

Rovelet-Lecrux A, Legallic S, Wallon D, Flaman JM, Martinaud O, Bombois S, RollinSillaire A, Michon A, Le Ber I, Pariente J, et al.; Investigators of the GMAJ Project (2012) A genome-wide study reveals rare CNVs exclusive to extreme phenotypes of Alzheimer disease. Eur J Hum Genet 20:613-617. 
Rudman D, Kutner MH, Redd SC II, Waters WC IV, Gerron GG, and Bleier J (1982) Hypocitraturia in calcium nephrolithiasis. J Clin Endocrinol Metab 55: $1052-1057$

Rusu V, Hoch E, Mercader JM, Tenen DE, Gymrek M, Hartigan CR, DeRan M, von Grotthuss M, Fontanillas P, Spooner A, et al.; MEDIA Consortium SIGMA T2D Consortium (2017) Type 2 diabetes variants disrupt function of SLC16A11 through two distinct mechanisms. Cell 170:199-212.e20.

Rutter GA and Chimienti F (2015) SLC30A8 mutations in type 2 diabetes. Diabetologia 58:31-36.

Ryan DH (2000) Use of sibutramine and other noradrenergic and serotonergic drugs in the management of obesity. Endocrine 13:193-199.

Saheki T, Iijima M, Li MX, Kobayashi K, Horiuchi M, Ushikai M, Okumura F, Meng XJ, Inoue I, Tajima A, et al. (2007) Citrin/mitochondrial glycerol-3-phosphate dehydrogenase double knock-out mice recapitulate features of human citrin deficiency. J Biol Chem 282:25041-25052.

Saheki T, Inoue K, Ono H, Katsura N, Yokogawa M, Yoshidumi Y, Furuie S, Kuroda E, Ushikai M, Asakawa A, et al. (2012) Effects of supplementation on food intake, body weight and hepatic metabolites in the citrin/mitochondrial glycerol-3-phosphate dehydrogenase double-knockout mouse model of human citrin deficiency. Mol Genet Metab 107:322-329.

Saheki T and Song YZ (2005) Citrin deficiency, in GeneReviews (Adam MP, Ardinger HH, Pagon RA, Wallace SE, Bean LJH, Stephens K, and Amemiya A eds), University of Washington, Seattle, WA

Saito M, Okamatsu-Ogura Y, Matsushita M, Watanabe K, Yoneshiro T, NioKobayashi J, Iwanaga T, Miyagawa M, Kameya T, Nakada K, et al. (2009) High incidence of metabolically active brown adipose tissue in healthy adult humans: effects of cold exposure and adiposity. Diabetes 58:1526-1531.

Samuel VT, Petersen KF, and Shulman GI (2010) Lipid-induced insulin resistance: unravelling the mechanism. Lancet 375:2267-2277.

Santer R, Kinner M, Lassen CL, Schneppenheim R, Eggert P, Bald M, Brodehl J, Daschner M, Ehrich JH, Kemper M, et al. (2003) Molecular analysis of the SGLT2 gene in patients with renal glucosuria. J Am Soc Nephrol 14:2873-2882.

Santer R, Kinner M, Schneppenheim R, Hillebrand G, Kemper M, Ehrich J, Swift P, Skovby F, and Schaub J (2000) The molecular basis of renal glucosuria: mutations in the gene for a renal glucose transporter (SGLT2). J Inherit Metab Dis 23:178.

Sasahara M, Nishi M, Kawashima H, Ueda K, Sakagashira S, Furuta H, Matsumoto E, Hanabusa T, Sasaki H, and Nanjo K (2004) Uncoupling protein 2 promoter polymorphism -866G/A affects its expression in beta-cells and modulates clinical profiles of Japanese type 2 diabetic patients. Diabetes 53:482-485.

Saxena R, Voight BF, Lyssenko V, Burtt NP, de Bakker PI, Chen H, Roix JJ, Kathiresan S, Hirschhorn JN, Daly MJ, et al.; Diabetes Genetics Initiative of Broad Institute of Harvard and MIT, Lund University, and Novartis Institutes of BioMedical Research (2007) Genome-wide association analysis identifies loci for type 2 diabetes and triglyceride levels. Science 316:1331-1336.

Scheen AJ (2015) Pharmacodynamics, efficacy and safety of sodium-glucose cotransporter type 2 (SGLT2) inhibitors for the treatment of type 2 diabetes mellitus. Drugs 75:33-59.

Schossig A, Bloch-Zupan A, Lussi A, Wolf NI, Raskin S, Cohen M, Giuliano F, Jurgens J, Krabichler B, Koolen DA, et al. (2017) SLC13A5 is the second gene associated with Kohlschütter-Tönz syndrome. J Med Genet 54:54-62.

Schrauwen P and Hesselink MK (2004) The role of uncoupling protein 3 in fatty acid metabolism: protection against lipotoxicity? Proc Nutr Soc 63:287-292.

Schrauwen P, Hoeks J, Schaart G, Kornips E, Binas B, Van De Vusse GJ, Van Bilsen M, Luiken JJ, Coort SL, Glatz JF, et al. (2003) Uncoupling protein 3 as a mitochondrial fatty acid anion exporter. FASEB J 17:2272-2274.

Schrauwen P, Mensink M, Schaart G, Moonen-Kornips E, Sels JP, Blaak EE, Russell AP, and Hesselink MK (2006) Reduced skeletal muscle uncoupling protein-3 content in prediabetic subjects and type 2 diabetic patients: restoration by rosiglitazone treatment. J Clin Endocrinol Metab 91:1520-1525.

Schrauwen P, Walder K, and Ravussin E (1999a) Human uncoupling proteins and obesity. Obes Res 7:97-105.

Schrauwen P, Xia J, Bogardus C, Pratley RE, and Ravussin E (1999b) Skeletal muscle uncoupling protein 3 expression is a determinant of energy expenditure in Pima Indians. Diabetes 48:146-149.

Schrauwen P, Xia J, Walder K, Snitker S, and Ravussin E (1999c) A novel polymorphism in the proximal UCP3 promoter region: effect on skeletal muscle UCP3 mRNA expression and obesity in male non-diabetic Pima Indians. Int $J$ Obes Relat Metab Disord 23:1242-1245.

Schwarz F, Karadeniz Z, Fischer-Rosinsky A, Willmes DM, Spranger J, and Birkenfeld AL (2015) Knockdown of Indy/CeNac2 extends Caenorhabditis elegans life span by inducing AMPK/aak-2. Aging (Albany NY) 7:553-567.

Scott DA and Fisher AM (1938) The insulin and the zinc content of normal and diabetic pancreas. J Clin Invest 17:725-728.

Scott LJ, Mohlke KL, Bonnycastle LL, Willer CJ, Li Y, Duren WL, Erdos MR, Stringham HM, Chines PS, Jackson AU, et al. (2007) A genome-wide association study of type 2 diabetes in Finns detects multiple susceptibility variants. Science 316:1341-1345.

Sekler I, Sensi SL, Hershfinkel M, and Silverman WF (2007) Mechanism and regulation of cellular zinc transport. Mol Med 13:337-343.

Servo C and Pitkänen E (1975) Variation in polyol levels in cerebrospinal fluid and serum in diabetic patients. Diabetologia 11:575-580.

Sesti G, Cardellini M, Marini MA, Frontoni S, D’Adamo M, Del Guerra S, Lauro D, De Nicolais P, Sbraccia P, Del Prato S, et al. (2003) A common polymorphism in the promoter of UCP2 contributes to the variation in insulin secretion in glucosetolerant subjects. Diabetes 52:1280-1283.

Shan Z, Bao W, Zhang Y, Rong Y, Wang X, Jin Y, Song Y, Yao P, Sun C, Hu FB, et al. (2014) Interactions between zinc transporter-8 gene (SLC30A8) and plasma zin concentrations for impaired glucose regulation and type 2 diabetes. Diabetes 63: 1796-1803.

Shubrook JH, Bokaie BB, and Adkins SE (2015) Empagliflozin in the treatment of type 2 diabetes: evidence to date. Drug Des Devel Ther 9:5793-5803.
Shulman GI and Helfand SL (2011) Indy knockdown in mice mimics elements of dietary restriction. Aging (Albany NY) 3:701.

Simino J, Shi G, Arnett D, Broeckel U, Hunt SC, and Rao DC (2011) Variants on chromosome 6p22.3 associated with blood pressure in the HyperGEN study: followup of FBPP quantitative trait loci. Am J Hypertens 24:1227-1233.

Sinasac DS, Moriyama M, Jalil MA, Begum L, Li MX, Iijima M, Horiuchi M, Robinson BH, Kobayashi K, Saheki T, et al. (2004) Slc25a13-knockout mice harbor metabolic deficits but fail to display hallmarks of adult-onset type II citrullinemia. Mol Cell Biol 24:527-536.

Sjöström L, Rissanen A, Andersen T, Boldrin M, Golay A, Koppeschaar HP, and Krempf M; European Multicentre Orlistat Study Group (1998) Randomised placebo-controlled trial of orlistat for weight loss and prevention of weight regain in obese patients. Lancet 352:167-172.

Sladek R, Rocheleau G, Rung J, Dina C, Shen L, Serre D, Boutin P, Vincent D, Belisle A, Hadjadj S, et al. (2007) A genome-wide association study identifies novel risk loci for type 2 diabetes. Nature 445:881-885.

Smidt K, Jessen N, Petersen AB, Larsen A, Magnusson N, Jeppesen JB, Stoltenberg M, Culvenor JG, Tsatsanis A, Brock B, et al. (2009) SLC30A3 responds to glucoseand zinc variations in beta-cells and is critical for insulin production and in vivo glucose-metabolism during beta-cell stress. PLoS One 4:e5684.

Smidt K, Pedersen SB, Brock B, Schmitz O, Fisker S, Bendix J, Wogensen L, and Rungby $J$ (2007) Zinc-transporter genes in human visceral and subcutaneous adipocytes: lean versus obese. Mol Cell Endocrinol 264:68-73.

Souza BM, Assmann TS, Kliemann LM, Gross JL, Canani LH, and Crispim D (2011) The role of uncoupling protein 2 (UCP2) on the development of type 2 diabetes mellitus and its chronic complications. Arq Bras Endocrinol Metabol 55:239-248.

Sreedhar A and Zhao Y (2017) Uncoupling protein 2 and metabolic diseases. Mitochondrion 34:135-140.

Stadler LKJ and Farooqi IS (2017) A new drug target for type 2 diabetes. Cell 170: 12-14.

Stanhope KL and Havel PJ (2010) Fructose consumption: recent results and their potential implications. Ann N Y Acad Sci 1190:15-24.

Stanley CA, Hale DE, Berry GT, Deleeuw S, Boxer J, and Bonnefont JP (1992) Brief report: a deficiency of carnitine-acylcarnitine translocase in the inner mitochondrial membrane. N Engl J Med 327:19-23.

Suhre K, Shin SY, Petersen AK, Mohney RP, Meredith D, Wägele B, Altmaier E, Deloukas P, Erdmann J, Grundberg E, et al.; CARDIoGRAM (2011) Human metabolic individuality in biomedical and pharmaceutical research. Nature 477:54-60.

Sun J, Aluvila S, Kotaria R, Mayor JA, Walters DE, and Kaplan RS (2010) Mitochondrial and plasma membrane citrate transporters: discovery of selective inhibitors and application to structure/function analysis. Mol Cell Pharmacol 2: 101-110.

Takagi H, Hagiwara S, Hashizume H, Kanda D, Sato K, Sohara N, Kakizaki S, Takahashi H, Mori M, Kaneko H, et al. (2006) Adult onset type II citrullinemia as a cause of non-alcoholic steatohepatitis. J Hepatol 44:236-239.

Tamaki M, Fujitani Y, Hara A, Uchida T, Tamura Y, Takeno K, Kawaguchi M, Watanabe T, Ogihara T, Fukunaka A, et al. (2013) The diabetes-susceptible gene SLC30A8/ZnT8 regulates hepatic insulin clearance. $J$ Clin Invest 123:4513-4524.

Tamaki M, Fujitani Y, Uchida T, Hirose T, Kawamori R, and Watada H (2009) Downregulation of ZnT8 expression in pancreatic $\beta$-cells of diabetic mice. Islets 1 : 124-128.

Taylor CG (2005) Zinc, the pancreas, and diabetes: insights from rodent studies and future directions. Biometals 18:305-312.

Tazawa S, Yamato T, Fujikura H, Hiratochi M, Itoh F, Tomae M, Takemura Y, Maruyama H, Sugiyama T, Wakamatsu A, et al. (2005) SLC5A9/SGLT4, a new $\mathrm{Na}+$-dependent glucose transporter, is an essential transporter for mannose, 1,5anhydro-D-glucitol, and fructose. Life Sci 76:1039-1050.

Tazawa Y, Kobayashi K, Ohura T, Abukawa D, Nishinomiya F, Hosoda Y, Yamashita M, Nagata I, Kono Y, Yasuda T, et al. (2001) Infantile cholestatic jaundice associated with adult-onset type II citrullinemia. J Pediatr 138:735-740.

Thevenon J, Milh M, Feillet F, St-Onge J, Duffourd Y, Jugé C, Roubertie A, Héron D, Mignot C, Raffo E, et al. (2014) Mutations in SLC13A5 cause autosomal-recessive epileptic encephalopathy with seizure onset in the first days of life. Am J Hum Genet 95:113-120.

Tise CG, Perry JA, Anforth LE, Pavlovich MA, Backman JD, Ryan KA, Lewis JP, O'Connell JR, Yerges-Armstrong LM, and Shuldiner AR (2016) From genotype to phenotype: nonsense variants in SLC13A1 are associated with decreased serum sulfate and increased serum aminotransferases. G3 (Bethesda) 6:2909-2918.

Toggenburger G, Kessler M, and Semenza G (1982) Phlorizin as a probe of the smallintestinal $\mathrm{Na}+$,D-glucose cotransporter: a model. Biochim Biophys Acta 688:557-571.

Traurig M, Hanson RL, Marinelarena A, Kobes S, Piaggi P, Cole S, Curran JE, Blangero J, Göring H, Kumar S, et al. (2016) Analysis of SLC16A11 variants in 12,811 American Indians: genotype-obesity interaction for type 2 diabetes and an association with RNASEK expression. Diabetes 65:510-519.

Triplitt C and Cornell S (2015) Canagliflozin treatment in patients with type 2 diabetes mellitus. Clin Med Insights Endocrinol Diabetes 8:73-81.

Tsai CW, Yang CC, Chen HL, Hwu WL, Wu MZ, Liu KL, and Wu MS (2006) Homozygous SLC25A13 mutation in a Taiwanese patient with adult-onset citrullinemia complicated with steatosis and hepatocellular carcinoma. J Formos Med Assoc 105:852-856.

Tuncay E, Bitirim CV, Olgar Y, Durak A, Rutter GA, and Turan B (2019) Zn ${ }^{2+}$. transporters ZIP7 and ZnT7 play important role in progression of cardiac dysfunction via affecting sarco(endo)plasmic reticulum-mitochondria coupling in hyperglycemic cardiomyocytes. Mitochondrion 44:41-52.

Tuncay E, Bitirim VC, Durak A, Carrat GRJ, Taylor KM, Rutter GA, and Turan B (2017) Hyperglycemia-induced changes in ZIP7 and ZnT7 expression cause $\mathrm{Zn}^{2+}$ release from the sarco(endo)plasmic reticulum and mediate ER stress in the heart. Diabetes 66:1346-1358.

Turk E, Zabel B, Mundlos S, Dyer J, and Wright EM (1991) Glucose/galactose malabsorption caused by a defect in the $\mathrm{Na}+$ /glucose cotransporter. Nature 350: 354-356. 
Udomsilp P, Saepoo S, Ittiwut R, Shotelersuk V, Dissayabutra T, Boonla C, and Tosukhowong P (2018) rs11567842 SNP in SLC13A2 gene associates with hypocitraturia in Thai patients with nephrolithiasis. Genes Genomics 40:965-972

Ung C, Sanchez AV, Shen L, Davoudi S, Ahmadi T, Navarro-Gomez D, Chen CJ, Hancock H, Penman A, Hoadley S, et al. (2017) Whole exome sequencing identification of novel candidate genes in patients with proliferative diabetic retinopathy. Vision Res 139:168-176.

Urano T, Shiraki M, Sasaki N, Ouchi Y, and Inoue S (2015) SLC25A24 as a novel susceptibility gene for low fat mass in humans and mice. J Clin Endocrinol Metab 100:E655-E663.

Urhammer SA, Dalgaard LT, Sørensen TI, Møller AM, Andersen T, Tybjaerg-Hansen A, Hansen T, Clausen JO, Vestergaard H, and Pedersen O (1997) Mutational analysis of the coding region of the uncoupling protein 2 gene in obese NIDDM patients: impact of a common amino acid polymorphism on juvenile and maturity onset forms of obesity and insulin resistance. Diabetologia 40:1227-1230.

Urhammer SA, Dalgaard LT, Sørensen TI, Tybjaerg-Hansen A, Echwald SM, Andersen T, Clausen JO, and Pedersen O (1998) Organisation of the coding exons and mutational screening of the uncoupling protein 3 gene in subjects with juvenile-onset obesity. Diabetologia 41:241-244.

Vallee BL and Falchuk KH (1993) The biochemical basis of zinc physiology. Physiol Rev 73:79-118.

Vallon V, Platt KA, Cunard R, Schroth J, Whaley J, Thomson SC, Koepsell H, and Rieg T (2011) SGLT2 mediates glucose reabsorption in the early proximal tubule. J Am Soc Nephrol 22:104-112.

van den Heuvel LP, Assink K, Willemsen M, and Monnens L (2002) Autosomal recessive renal glucosuria attributable to a mutation in the sodium glucose cotransporter (SGLT2). Hum Genet 111:544-547.

van der Harst P, Bakker SJ, de Boer RA, Wolffenbuttel BH, Johnson T, Caulfield MJ, and Navis G (2010) Replication of the five novel loci for uric acid concentrations and potential mediating mechanisms. Hum Mol Genet 19:387-395.

van Hasselt PM, Ferdinandusse S, Monroe GR, Ruiter JP, Turkenburg M, Geerlings MJ, Duran K, Harakalova M, van der Zwaag B, Monavari AA, et al. (2014) Monocarboxylate transporter 1 deficiency and ketone utilization. $N$ Engl J Med 371: 1900-1907.

van Marken Lichtenbelt WD, Vanhommerig JW, Smulders NM, Drossaerts JM, Kemerink GJ, Bouvy ND, Schrauwen P, and Teule GJ (2009) Cold-activated brown adipose tissue in healthy men. $N$ Engl J Med 360:1500-1508.

Vidal-Puig AJ, Grujic D, Zhang CY, Hagen T, Boss O, Ido Y, Szczepanik A, Wade J, Mootha V, Cortright R, et al. (2000) Energy metabolism in uncoupling protein 3 gene knockout mice. J Biol Chem 275:16258-16266.

Virtanen KA, Lidell ME, Orava J, Heglind M, Westergren R, Niemi T, Taittonen M, Laine J, Savisto NJ, Enerbäck S, et al. (2009) Functional brown adipose tissue in healthy adults. $N$ Engl J Med 360:1518-1525.

Visser WE, Friesema EC, and Visser TJ (2011) Minireview: thyroid hormone transporters: the knowns and the unknowns. Mol Endocrinol 25:1-14.

von Loeffelholz C, Döcke S, Lock JF, Lieske S, Horn P, Kriebel J, Wahl S, Singmann $\mathrm{P}$, de Las Heras Gala T, Grallert H, et al. (2017a) Increased lipogenesis in spite of upregulated hepatic 5'AMP-activated protein kinase in human non-alcoholic fatty liver. Hepatol Res 47:890-901.

von Loeffelholz C, Lieske S, Neuschäfer-Rube F, Willmes DM, Raschzok N, Sauer IM König J, Fromm MF, Horn P, Chatzigeorgiou A, et al. (2017b) The human longevity gene homolog INDY and interleukin-6 interact in hepatic lipid metabolism. Hepatology 66:616-630.

Wada M, Shimada A, and Fujita T (2006) Functional characterization of $\mathrm{Na}+$-coupled citrate transporter $\mathrm{NaC} 2 / \mathrm{NaCT}$ expressed in primary cultures of neurons from mouse cerebral cortex Brain Res 1081:92-100.

Walder K, Norman RA, Hanson RL, Schrauwen P, Neverova M, Jenkinson CP, Easlick J, Warden CH, Pecqueur C, Raimbault S, et al. (1998) Association between uncoupling protein polymorphisms (UCP2-UCP3) and energy metabolism/obesity in Pima Indians. Hum Mol Genet 7:1431-1435.

Wang PY, Neretti N, Whitaker R, Hosier S, Chang C, Lu D, Rogina B, and Helfand SL (2009) Long-lived Indy and calorie restriction interact to extend life span. Proc Natl Acad Sci USA 106:9262-9267.

Weeke LC, Brilstra E, Braun KP, Zonneveld-Huijssoon E, Salomons GS, Koeleman BP, van Gassen KL, van Straaten HL, Craiu D, and de Vries LS (2017) Punctate white matter lesions in full-term infants with neonatal seizures associated with SLC13A5 mutations. Eur J Paediatr Neurol 21:396-403.

Wenzlau JM, Juhl K, Yu L, Moua O, Sarkar SA, Gottlieb P, Rewers M, Eisenbarth GS, Jensen J, Davidson HW, et al. (2007) The cation efflux transporter ZnT8 (Slc30A8) is a major autoantigen in human type 1 diabetes. Proc Natl Acad Sci USA 104:17040-17045.
Wijesekara N, Dai FF, Hardy AB, Giglou PR, Bhattacharjee A, Koshkin V, Chimienti F, Gaisano HY, Rutter GA, and Wheeler MB (2010) Beta cell-specific Znt8 deletion in mice causes marked defects in insulin processing, crystallisation and secretion. Diabetologia 53:1656-1668.

Williams AL, Jacobs SB, Moreno-Macías H, Huerta-Chagoya A, Churchhouse C, MárquezLuna C, García-Ortíz H, Gómez-Vázquez MJ, Burtt NP, Aguilar-Salinas CA, et al.; SIGMA Type 2 Diabetes Consortium (2014) Sequence variants in SLC16A11 are a common risk factor for type 2 diabetes in Mexico. Nature 506:97-101.

Willmes DM and Birkenfeld AL (2013) The role of INDY in metabolic regulation. Comput Struct Biotechnol J 6:e201303020.

Willmes DM, Helfand SL, and Birkenfeld AL (2016) The longevity transporter mIndy (Slc13a5) as a target for treating hepatic steatosis and insulin resistance. Aging (Albany NY) 8:208-209.

Willmes DM, Kurzbach A, Henke C, Schumann T, Zahn G, Heifetz A, Jordan J, Helfand SL, and Birkenfeld AL (2018) The longevity gene INDY (I'm Not Dead Yet) in metabolic control: potential as pharmacological target. Pharmacol Ther 185:1-11.

Winegrad AI and Clements RS Jr (1971) Diabetic ketoacidosis. Med Clin North Am 55:899-911.

Wiviott SD, Raz I, Bonaca MP, Mosenzon O, Kato ET, Cahn A, Silverman MG, Zelniker TA, Kuder JF, Murphy SA, et al.; DECLARE-TIMI 58 Investigators (2019) Dapagliflozin and cardiovascular outcomes in type 2 diabetes. $N$ Engl J Med 380:347-357.

Wong WP, Allen NB, Meyers MS, Link EO, Zhang X, MacRenaris KW, and El Muayed M (2017) Exploring the association between demographics, SLC30A8 genotype, and human islet content of zinc, cadmium, copper, iron, manganese and nickel. Sci Rep 7:473.

Wright E, Martin MG, and Turk E (2001) Familial glucose-galactose malabsorption and hereditary renal glycosuria, in Metabolic Basis of Inherited DiseaseScriver CR Beaudet AL, Sly WS, and Valle D eds) pp 4891-4908, McGraw Hill, New York.

Wright EM (2013) Glucose transport families SLC5 and SLC50. Mol Aspects Med 34 183-196.

Wright EM, Loo DD, and Hirayama BA (2011) Biology of human sodium glucose transporters. Physiol Rev 91:733-794.

Yamada T, Katagiri H, Ishigaki Y, Ogihara T, Imai J, Uno K, Hasegawa Y, Gao J, Ishihara H, Niijima A, et al. (2006) Signals from intra-abdominal fat modulate insulin and leptin sensitivity through different mechanisms: neuronal involvement in food-intake regulation. Cell Metab 3:223-229.

Yamanouchi T, Akanuma Y, Toyota T, Kuzuya T, Kawai T, Kawazu S, Yoshioka S, Kanazawa Y, Ohta M, Baba S, et al. (1991) Comparison of 1,5-anhydroglucitol, $\mathrm{HbA1c}$, and fructosamine for detection of diabetes mellitus. Diabetes 40:52-57.

Yan HM, Hu H, Ahmed A, Feng BB, Liu J, Jia ZJ, and Wang H (2017) Carnitineacylcarnitine translocase deficiency with c.199-10 $\mathrm{T}>\mathrm{G}$ and novel c.1A $>\mathrm{G}$ mutation: two case reports and brief literature review. Medicine (Baltimore) 96:e8549.

Yodoya E, Wada M, Shimada A, Katsukawa H, Okada N, Yamamoto A, Ganapathy V, and Fujita T (2006) Functional and molecular identification of sodium-coupled dicarboxylate transporters in rat primary cultured cerebrocortical astrocytes and neurons. $J$ Neurochem 97:162-173.

Zelniker TA, Wiviott SD, Raz I, Im K, Goodrich EL, Bonaca MP, Mosenzon O, Kato ET, Cahn A, Furtado RHM, et al. (2019) SGLT2 inhibitors for primary and secondary prevention of cardiovascular and renal outcomes in type 2 diabetes: a systematic review and meta-analysis of cardiovascular outcome trials. Lancet 393:31-39.

Zhang CY, Baffy G, Perret P, Krauss S, Peroni O, Grujic D, Hagen T, Vidal-Puig AJ, Boss O, Kim YB, et al. (2001) Uncoupling protein-2 negatively regulates insulin secretion and is a major link between obesity, beta cell dysfunction, and type 2 diabetes. Cell 105:745-755.

Zhang M, Wang M, and Zhao ZT (2014) Uncoupling protein 2 gene polymorphisms in association with overweight and obesity susceptibility: a meta-analysis. Meta Gene 2:143-159

Zhang Y, Zhang Y, Sun K, Meng Z, and Chen L (2019) The SLC transporter in nutrient and metabolic sensing, regulation, and drug development. J Mol Cell Biol 11:1-13.

Zhao S, Chu Y, Zhang Y, Zhou Y, Jiang Z, Wang Z, Mao L, Li K, Sun W, Li P, et al. (2019a) Linifanib exerts dual anti-obesity effect by regulating adipocyte browning and formation. Life Sci 222:117-124.

Zhao Y, Feng Z, Zhang Y, Sun Y, Chen Y, Liu X, Li S, Zhou T, Chen L, Wei Y, et al. (2019b) Gain-of-function mutations of SLC16A11 contribute to the pathogenesis of type 2 diabetes. Cell Rep 26:884-892.e4.

Zinman B, Wanner C, Lachin JM, Fitchett D, Bluhmki E, Hantel S, Mattheus M, Devins T, Johansen OE, Woerle HJ, et al.; EMPA-REG OUTCOME Investigators (2015) Empagliflozin, cardiovascular outcomes, and mortality in type 2 diabetes. N Engl J Med 373:2117-2128. 